\title{
Examining Phylogenetic Relationships Among Gibbon Genera Using Whole Genome Sequence Data Using an Approximate Bayesian Computation Approach
}

\begin{abstract}
Krishna R. Veeramah, ${ }^{*,+}$ August E. Woerner, * Laurel Johnstone, ${ }^{*}$ Ivo Gut, ${ }^{\dagger}$ Marta Gut, ${ }^{*}$ Tomas Marques-Bonet, ${ }^{\ddagger, \S}$ Lucia Carbone, ${ }^{* *}$ Jeff D. Wall, ${ }^{\dagger+}$ and Michael F. Hammer ${ }^{*, 1}$ *Arizona Research Laboratories Division of Biotechnology, University of Arizona, Tucson, Arizona 85721, 'Department of Ecology and Evolution, Stony Brook University, Stony Brook, New York 11794, ${ }^{\ddagger}$ Centro Nacional de Analisis Genomico, 08028 Barcelona,

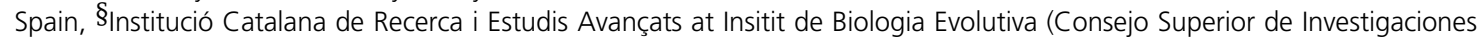
Científicas/Universitat Pompeu Fabra), 08003 Barcelona, Spain, **Department of Behavioral Neuroscience, Oregon Health and Science University, Portland, Oregon 97239, and ${ }^{+\dagger}$ Institute for Human Genetics, University of California, San Francisco, California 94143
\end{abstract}

\begin{abstract}
Gibbons are believed to have diverged from the larger great apes 16.8 MYA and today reside in the rainforests of Southeast Asia. Based on their diploid chromosome number, the family Hylobatidae is divided into four genera, Nomascus, Symphalangus, Hoolock, and Hylobates. Genetic studies attempting to elucidate the phylogenetic relationships among gibbons using karyotypes, mitochondrial DNA (mtDNA), the $Y$ chromosome, and short autosomal sequences have been inconclusive. To examine the relationships among gibbon genera in more depth, we performed second-generation whole genome sequencing (WGS) to a mean of $\sim 15 \times$ coverage in two individuals from each genus. We developed a coalescent-based approximate Bayesian computation (ABC) method incorporating a model of sequencing error generated by high coverage exome validation to infer the branching order, divergence times, and effective population sizes of gibbon taxa. Although Hoolock and Symphalangus are likely sister taxa, we could not confidently resolve a single bifurcating tree despite the large amount of data analyzed. Instead, our results support the hypothesis that all four gibbon genera diverged at approximately the same time. Assuming an autosomal mutation rate of $1 \times 10^{-9} /$ site/year this speciation process occurred $~ 5$ MYA during a period in the Early Pliocene characterized by climatic shifts and fragmentation of the Sunda shelf forests. Whole genome sequencing of additional individuals will be vital for inferring the extent of gene flow among species after the separation of the gibbon genera.
\end{abstract}

KEYWORDS approximate Bayesian computation; gibbon species; rapid radiation; whole genome sequences

HE family Hylobatidae, commonly known as gibbons, is believed to have diverged from the larger great apes $\sim 16.8$ MYA (Carbone et al. 2014). Sometimes known as small apes, gibbons demonstrate substantial morphological differentiation from the great apes; their much smaller bodies are highly adapted to an arboreal mode of locomotion in the rainforests

Copyright (C) 2015 by the Genetics Society of America

doi: 10.1534/genetics.115.174425

Manuscript received January 12, 2015; accepted for publication March 4, 2015 published Early Online March 12, 2015.

Supporting information is available online at http://www.genetics.org/lookup/suppl/ doi:10.1534/genetics.115.174425/-/DC1.

${ }^{1}$ Corresponding author: ARL Division of Biotechnology, Keating Building $111 \mathrm{~K}$,

University of Arizona, Tucson, AZ 85721. E-mail: mfh@email.arizona.edu of Southeast Asia. They also demonstrate very little sexual dimorphism that may, in part, be related to their generally monogamous mating patterns (Fuentes 2000) (although some gibbon species develop differences in coat color at sexual maturity).

Each species demonstrates distinct "call" and "song" types (Geissmann 2002); however, attempts to classify gibbon species and genera based solely on morphological features have been problematic (Mootnick 2006). Primarily on the basis of their karyotypes, gibbons are now divided into four major genera, with Nomascus, Symphalangus, Hylobates, and Hoolock each possessing 52, 50, 44, and 38 diploid chromosomes, respectively. While many genetic studies have been performed, including a number based on karyotypes (Müller et al. 2003), 
mitochondrial DNA (mtDNA) (Hayashi et al. 1995; Takacs et al. 2005; Monda et al. 2007; Whittaker et al. 2007; Matsudaira and Ishida 2010; Van Ngoc et al. 2010), Y chromosomes (Chan et al. 2012), Arthrobacter luteus (ALU) repeats (Meyer et al. 2012), and short stretches of autosomal sequence (Kim et al. 2011; Wall et al. 2013), the phylogenetic relationships among the four gibbon genera remain unresolved, with at least seven different topologies being supported by different data.

A recent study examined $\sim 1.5 \mathrm{Mb}$ of orthologous autosomal sequence generated by second-generation sequencing from one individual representing each of the four genera (Wall et al. 2013). This study, too, was inconclusive and suggested that the gibbon genealogy demonstrates substantial incomplete lineage sorting (ILS). However, the experimental design was limited by the lack of a suitable reference genome (short reads were aligned to highly divergent human hg19 assembly). To examine the species tree relationships among gibbons, as well as estimate key demographic parameters such as the time when the various gibbon genera diverged, we generate whole genome sequence data from eight individuals representing all four gibbon genera and utilize the newly released gibbon (nomLeu1) reference genome (Carbone et al. 2014) for mapping and variant calling. Then we apply a coalescent-based ABC approach that can handle large amounts of sequence data and that corrects for potential sequencing error and reference genome mapping bias.

\section{Materials and Methods}

\section{Second-generation sequencing}

Blood and tissues were obtained in agreement with protocols reviewed and approved by the Gibbon Conservation Center. More details on all aspects of the methods are provided in Supporting Information. DNA was extracted from blood or cell lines, and paired-end libraries were prepared with the Illumina TruSeq chemistry. Libraries were shotgun sequenced on the HiSeq 2000 platform, generating $2 \times 100$-bp reads. Multiple runs were performed to generate a minimum of $10 \times$ mean coverage on each sample after all postprocessing. Mean coverage ranged from $11.5 \times$ to $19.5 \times$. Exome capture using the TruSeq Exome Enrichment kit (Illumina) was also performed on one $N$. leucogenys (NLE) sample (Vok, $116 \times$ coverage) and one species syndactylus (SSY) sample (Monty, $64 \times$ coverage)

\section{Read mapping and variant calling}

Trimmed reads from the shotgun sequencing were aligned to nomLeu1 with Stampy (v. 1.0.17) (Lunter and Goodson 2011). For the two NLE samples, Stampy was used in its "hybrid mode" where alignment with Burrows-Wheeler Aligner (BWA) (v. 0.5.9) (Li and Durbin 2009) is attempted first. A substitution rate of 0.001 was specified, along with BWA minimum seed length of 2 , fraction of missing alignments 0.0001 , and quality threshold 10 . For the non-NLE samples, Stampy was used with a substitution rate of 0.015 (Kim et al. 2011). Local realignment at indel sites was performed with the Genome Analysis Toolkit (GATK, v. 1.4-37) (McKenna et al.
2010; Depristo et al. 2011). PCR duplicates were removed with samtools. GATK UnifiedGenotyper was run separately on the two samples from each genus and single nucleotide variants (SNVs) and indels with a quality score of at least 50 were retained to create a mask of variant sites to be excluded from base quality score recalibration. The GATK indel realignment tool was run again to standardize alignment of indels across all samples. UnifiedGenotyper from GATK version 2.1-11 (to allow multiallelic calling) was used to produce a final set of SNVs and indels. Each site was annotated with the consensus quality score of the nomLeul reference sequence. Exome sequencing data were processed separately from the shotgun data but using the same bioinformatic pipeline (more details can be found in Supporting Information).

\section{Masking of the gibbon reference genome for downstream analysis}

The nomLeu1 genome is composed of 17,968 contigs, ranging in size from 2496 bases to $\sim 74 \mathrm{Mb}$. As small loci may be compressed and represent duplications in the gibbon genome that have not been properly separated during the assembly process, we masked out all scaffolds $<1 \mathrm{Mb}$ in length, yielding 273 scaffolds that span $\sim 2.73 \mathrm{~Gb}$. University of California Santa Cruz (UCSC)'s gibbon-human pairwise alignments were used to identify nonautosomal sequence. Specifically, gibbon loci that aligned to human X, Y, or M in UCSC's "net" alignments (Kent et al. 2003) were masked, along with locations in the gibbon genome that were not primary alignments to locations in the human genome. Further, locations where the gibbon reference quality was below a phred quality of 50 repeats [identified by Tandem Repeat Finder (Benson 1999) or by RepeatMasker (Smit et al. 1996)], LAVA elements identified in Carbone et al. (2014), copy number variants (CNV) with an estimated ploidy $>2.5$ in any sample (also identified in Carbone et al. 2014), infinite site violations, positions where any sample has less than $<7 \times$ coverage, or more than their 95th percentile read depth, and bases within $3 \mathrm{bp}$ of any indel called were excluded, unless otherwise specified, from downstream analysis.

\section{Profiling of sequencing errors}

As our WGS coverage is $\sim 15 \times$ per sample, it is likely that some observed genotype calls will not reflect the true underlying genotypes, either because of a combination of low coverage and errors in the sequencing reads or as a result of choices in the bioinformatic processing (e.g., variant quality score thresholds). Therefore, we compared genotype calls between the WGS and whole exome sequencing (WES) data at the same genomic positions to profile errors present in our medium coverage WGS, assuming high coverage WES reflects a "truth set." Separate profiles were constructed for Vok and Monty. These profiles were then used (a) as training data to find a set of high confidence SNPs variable across all eight gibbon samples using machine learning (ML) methods, and (b) to stochastically model error processes in our subsequent $\mathrm{ABC}$ analysis. 
Our profiling and modeling of errors made the following simplifying assumptions: (1) after masking, any WES site with $30 \times \leq$ coverage $\leq 200 \times$ is called without error and reflects the truth dataset and all other bases are ignored, (2) per site read depth and mapping bias (which we naively model by noting whether the sample belongs to the same taxon as the reference or not) account for all genotyping errors observed, and (3) all false negatives (i.e., SNPs present in the WES, but not present in the whole genome data) are singletons. We profiled errors between the WES truth set and WGS for a given target sample (Vok or Monty) via two categories: errors involving singleton polymorphisms (defined with respect to the nomLeu1 reference), and genotyping errors when the polymorphism is segregating with a nonreference allele present in two or more chromosomes.

For the former category, we recorded the number of singleton calls in our WGS data vs. the number in the WES truth set. For a site to be considered a singleton, a single nonreference allele must be present in either the WGS, the WES truth set, or both for the target sample, and it must not have been observed in any other sample. Singleton sites that agree and disagree between the WGS and WES truth sets are considered as "correct" and "incorrect," respectively.

For the latter category (i.e., any site that is segregating in either the WGS, the WES truth set, or both, but is not defined as a singleton as described above) we created $3 \times 3$ confusion matrices over the set of genotype calls (reference homozygous, heterozygous, alternative homozygous) to describe all nine possible WGS $v s$. WES truth set genotype calls for the target sample. The diagonals of these confusion matrices reflect sites with concordant calls between the WGS $v s$. WES truth set (i.e., they are correct) and off-diagonals represent discordance, and thus potential errors (i.e., incorrect). For example, the sum of the middle column will represent all heterozygous sites in the WES truth set. The middle element of this column represents sites that were also called heterozygous in the WGS data (i.e., correctly called sites). The top (bottom) element represents a genotyping error in the WGS data where the site is truly heterozygous but was called homozygous reference (alternate).

\section{Finding accurately called segregating sites}

ML classification techniques, such as variant quality score recalibration, have been successfully used to find a subset of sites that are predicted to be truly segregating in a sample. However, the authors know of no technique that has been used to predict whether or not individual genotypes have been correctly called, and as such downstream methods that presume that the genotypes are correct when they are in fact incorrect may suffer accordingly. To this end we developed an ML classification protocol to find a set of segregating sites where every genotype within is predicted to be correct for use in our principal components analysis. Broadly, this protocol uses the comparison of the WGS and WES truth set to train several largely disparate classifiers. The classifiers are then used to predict the accuracy of individual genotypes across the genome. We note that this protocol may introduce some level of bias with respect to the agglomerative properties of sites (owing to the increased difficulty in calling heterozygous $v s$. homozygous genotypes) as opposed to individual genotypes, and as such this approach would be undesirable for evaluating, say, the site frequency spectrum.

More specifically, the ML suite Weka version 3.6.8 (Hall et al. 2008) was used to classify the WGS genotype data at all called segregating sites, with the aim of finding a subset of very high quality sites. Using the definition of correct from our profiling of errors, we collected the set of all genotypes that were incorrectly called in the genome, and a random and equally sized sampling of genotypes that were called correctly for both our NLE and our non-NLE (SSY) sample. A variety of features from the GATK output (see Supporting Information for the entire list) as well as whether the call is from the NLE or the non-NLE sample, and the combined $P$-value of the distribution of read depths observed at the site were used in the ML analysis. Using the various features, we generated a training set and evaluated the performance of a variety of classifiers using 10-fold cross-validation. Four techniques-multilayer perceptron, ridor, rotation forest, and classification by regression-showed reasonable performance (75-85\% accuracy). After various optimization procedures, we classified a genotype call as correct if all four classifiers predicted that the genotype was correct, and we classified a site as correct if all genotypes at a site were classified as correct. Principle Components Analysis (PCA) was performed using smartpca (Patterson et al. 2006) and visualized using R.

\section{$A B C$ analysis}

Our ABC framework was designed to (a) identify the most likely species topology for the four gibbon genera that underwent WGS and (b) estimate key parameters of the gibbon speciation process (specifically effective population sizes and divergence times) (more detail can be found in the Supporting Information).

Data: $\mathrm{ABC}$ analysis was performed on two datasets containing independent loci of small enough length such that intra and interlocus recombination could reasonably be ignored. Set 1 included 12,413 nongenic loci consisting of $1 \mathrm{~kb}$ of total callable sequence across a contiguous stretch of no more than $3 \mathrm{~kb}$ separated by at least $50 \mathrm{~kb}$ and at least $50 \mathrm{~kb}$ from the nearest exon. Set 2 included 11,323 genic loci consisting of $200 \mathrm{bp}$ of total callable sequence across a contiguous stretch of no more than $4 \mathrm{~kb}$ separated by at least $1 \mathrm{~kb}$ (this distance will likely violate our assumption of independence but increasing this distance substantially decreased the number of usable loci and thus reduced the accuracy and precision of our inference to a greater extent), with an allowance of a maximum of 100 bp of the locus lying adjacent to an exon and the rest lying in the exon (Figure S1). In addition to the masks and coverage filters described above, we also masked CpG consistent sites as well as conserved phastCons (Siepel et al. 2005) elements inferred from primate genomes with a further 100 bp padding 
either side of the element. Variant sites were polarized against the aligned human reference genome, hg19.

Phylogeny models and parameter priors: We treated all possible phylogenetic relationships among the four gibbon genera as distinct models (including, where applicable, the true polytomy model). The models are described by two classes of parameters, mean population nucleotide diversity, $\theta$, and branch lengths, $\tau$, in units of expected number of substitutions (thus mutation rates per site per generation do not need to be explicitly stated during the analysis). Priors ranged between 0.0001 and 0.03 for all $\theta$ and $\tau$ parameters (a justification for these prior ranges is given in Supporting Information). Unless otherwise stated, all prior distributions for all demographic parameters $(\theta$ and $\tau)$ are all uniformly distributed on a $\log _{10}(\times)$ scale.

Simulations: Coalescent simulations of demographic models and parameters were performed using a version of ms (Hudson 2002) modified for Python that allowed fast parallel processing to allow us to efficiently simulate the thousands of loci seen in our observed data. To account for mutation rate heterogeneity among loci, we estimated relative sequence divergence for all loci, taking the average sequence divergence for each of the eight gibbon individuals from hg19. These individual locus estimates were then normalized around a mean of 1 , allowing us to follow the approach of Rannala and Yang (2003) and scale $\theta$ for each individual locus in our demographic simulations.

Stochastic error modeling: We used the error profiles for the singleton and nonsingleton categories described above in Vok and Monty to construct an error model $E=<S, M>$ for a particular sample that could transform perfectly correct data generated by coalescent simulations into data reflective of the error processes that are likely to have occurred during whole genome sequencing and postprocessing. We found that with our bioinformatic pipeline, the total number of observed singletons was always less than or equal to the true number. Therefore $S$ was calculated as the proportion of missing singletons, or the probability of not calling a true singleton in the WGS data. During a coalescent simulation of genetic data, $S$ reflects the rate at which true singletons will be hidden or dropped and the genotype called as homozygous reference. To construct $M$, we took the $3 \times 3$ confusion matrix generated for nonsingletons and divided the number in each element of the matrix by the sum of all elements within their respective columns. During a simulation of genetic data, for any site not classed as a true singleton but still segregating, the values within a particular column of $M$ reflect the probabilities of a multinomial distribution that determines the rate that a true genotype of a particular type will be transformed to one of the two other genotypes or stay the same.

To apply our error correction to (a) nonexome regions in the two target samples, and (b) nonexome regions in the other six samples for which there was no WES, we constructed separate $E$ models for each read depth $\geq 7 \times$ (i.e., we constructed $E_{i}$, the estimated error rate at a particular read-depth i). This allowed us to construct an overall $E$ model for a particular sample, regardless of whether it was one of the two target samples or not, by taking a weighted average of $E_{i}$, with weights determined by the empirical distribution of read depths at the specific regions of interest. The $E_{i}$ models for Vok and Monty were used for NLE and non-NLE samples, respectively, to take into account any potential mapping biases.

Ancestral state misidentification adjustment: The $2 \%$ ancestral state misidentification was incorporated into simulations by calculating the expected number of sites to experience a mutation along the hg19 lineage for each locus (1000 bp $\times 2 \%=20$ sites). The number of sites to actually "flip" (i.e., assign the wrong ancestral state) for each locus during a simulation is drawn from a Poisson distribution with this mean. These sites are then randomly assigned to a position along the locus with equiprobability, though only positions that are found to segregate among the gibbon chromosomes need to be flipped computationally.

Summary statistics: We computed the following summary statistics to describe the observed and simulated data for every pair of populations across all loci: mean number of shared derived polymorphisms, mean number of private derived polymorphisms in each population, and the mean number of private fixed sites in each population. We also explored including the variance of these summary statistics across all loci but found they added little to our ability to infer parameters in the model while contributing more noise to the partial least squares (PLS) transformation and reduced the proportion of correctly inferred simulated topologies using simulated pseudo-observed data.

Inference: We used the logistic regression (LR) method previously described (Fagundes et al. 2007) to perform model choice. When estimating model parameters, we utilized ABCtoolbox (Wegmann et al. 2010), which implements a general linear model (GLM) adjustment (Leuenberger and Wegmann 2010) on retained simulations. Before ABC analysis for parameter inference, the full set of summary statistics was transformed into PLS components (Wegmann et al. 2009) and we used the change in root mean square error (RMSE) to guide the choice of number of components. The $1 \%$ of simulations closest to the observed data were retained for the GLM (parameter estimation) and LR (model choice) adjustments.

\section{G-PhoCS analysis}

The Markov chain Monte Carlo (MCMC) Bayesian coalescentbased method described by Gronau et al. (2011) was performed using the software G-PhoCS to estimate $\theta$ and $\tau$ values for a bifurcating tree (we ignored the effect of migration). On this occasion, we included a human haploid sequence (hg19) as an outgroup for the overall gibbon phylogeny (rather than just to infer the ancestral state as in the $A B C$ analysis). The same 12,431 1-kb loci and the bifurcating species tree with the highest posterior probability from the $\mathrm{ABC}$ analysis described above 
were utilized and the mutation rate was fixed individually for each locus as above using the normalized divergence values. The gamma prior for $\theta$ was set to be relatively broad and the same for all present and ancestral populations with shape, $\alpha=$ 2 and rate, $\beta=1000$. Gamma priors for $\tau$ were also set to be relatively broad, with the $\alpha$ value always 2 . However, either (a) $\beta$ was set as 200 for all $\tau$-values or (b) individual $\beta$-values were set for each $\tau$ such that the mean value reflected rough estimates from the $\mathrm{ABC}$ analysis or the human/gibbon split time from Carbone et al. (2014) (Table S1). We ran three independent MCMC chains for both prior settings a and b. We allowed 10,000 samples as burn-in followed by 100,000 samples for estimating parameters. The Markov chain converged to stationarity much quicker than the utilized burn-in period, and all six runs converged to the same stationary distribution. Results were processed using the software Tracer (http://tree.bio.ed.ac.uk/ software/tracer/).

\section{Results}

\section{Second generation sequencing and validation}

We performed second generation WGS on two individuals (one male and one female) from each of the four gibbon genera (Table 1). For our Nomascus samples, represented by the species leucogenys (NLE, the northern white-cheeked gibbon), the two individuals examined differed from the (NCBI Project 13975 GCA_000146795.1) nomLeu1 reference genome. For our Hylobates samples (the most diverse genus with 13 species), we examined one individual each from the $H$. moloch (HMO, Javan gibbon) and $H$. pileatus (HPI, pileated gibbon). Our Symphalangus sample is represented by two individuals from the species syndactylus (SSY, Siamang gibbon). It is important to point out that the two Hoolock samples from the leuconedys species (HLE, Eastern hoolock gibbon) represent the only wild-born individuals present in the study, whereas all other individuals were captive born (i.e., offspring of individuals living in zoos). We also mention that matings between different gibbon species (and even different genera) are known to result in viable offspring in captivity (Myers and Shafer 1979; Mootnick 2006; Hirai et al. 2007). If any of the individuals in our sample are indeed hybrids between different species, our analysis may be affected in unexpected ways.

After postprocessing the sequence data, we obtained a mean coverage of $15 \times(\min =11.5 \times, \max =19.5 \times)$ (Figure $\mathrm{S} 2)$. As previous work has indicated a relatively high divergence between gibbon genera, we attempted to incorporate potential reference bias into our postprocessing by utilizing a higher substitution rate $(1.5 \%)$ when mapping sequence reads for nonNLE samples, and by using a hybrid mapper, Stampy (Lunter and Goodson 2011) to increase sensitivity. To validate our variant calling, we performed high coverage WES on one NLE individual and one non-NLE sample (the male SSY sample). Mean coverage for WES data were $116 \times$ (compared with $14 \times$ for WGS data) and $64 \times$ (compared with $13 \times$ for WGS data), respectively. Human-based exome capture has been shown to be effective in primates as diverged from humans as macaques (Jin et al. 2012). Utilizing only exome calls with coverage between $30 \times$ and $200 \times$ we found slightly greater concordance between the WGS and WES data for the NLE (99.6\%) vs. non-NLE samples (99.4\%) (Table S2). Noticeably when only examining singleton variants, calling was markedly better in the reference taxa ( $\sim 99 \%$ of exome-called sites identified in the WGS data) than in the nonreference taxa ( 96\%), suggesting reference biases may still exist in our data for rare variants in nonreference taxa.

\section{Genetic diversity among gibbon genera}

Within genera diversity, assessed for this dataset by Carbone et al. (2014), demonstrated that NLE samples had the highest level of nucleotide diversity $\left(\pi \sim 2.2 \times 10^{-3}\right)$, while values as low as $\sim 7.3 \times 10^{-4}$ were observed in the HPI sample. Nucleotide diversity for the HMO sample was also relatively high at $\sim 1.7 \times 10^{-3}$, followed by SSY $\left(\sim 1.4 \times 10^{-3}\right)$, and then the two wild-born HLE $\left(\sim 8 \times 10^{-3}\right)$. By way of comparison, $\pi$ ranges from $\sim 0.5-1.0 \times 10^{-3}$ in humans, $1.8 \times 10^{-3}$ in western lowland gorillas, and $2.3 \times 10^{-3}$ in Sumatran orangutans (Prado-Martinez et al. 2013). To examine the relative levels of genetic differentiation among the gibbon genera we performed PCA on the individual samples. For this analysis we examined diallelic SNPs called in all individuals. High-quality SNPs were identified by using concordance with the WES data to train a ML algorithm to predict highly confident genotype calls across the whole genome and in samples that did not undergo WES. In addition, to ensure independence of SNPs, we randomly selected sites that were separated by at least $100 \mathrm{~kb}$ when on the same scaffold. This resulted in a dataset of 25,531 high-quality genome-wide independent SNPs. The first four principal components accounted for 40.2, 31.2, 24.6, and $3.5 \%$ of the variation, respectively (Figure S3, A and B). The four genera showed substantial genetic differentiation and were clearly separated in the PCA plot in the first two components, though no clear intergenera phylogenetic relationship emerged. Individuals from the same species showed high similarity suggesting limited intergenera hybridization or contamination. The two Hylobates species could be clearly distinguished in PC4. We were also able to reproduce the same patterns when only using a random subset of $\sim 200$ SNPs (Figure S3, C and D), suggesting it may be possible to perform relatively low coverage shotgun sequencing from a number of different gibbon species and use a similar approach to this in order to identify a small yet powerful set of species-specific SNPs. This could be particularly important for management of gibbons in zoos when it can often be difficult to distinguish different species or even genera based on fur alone, sometimes leading to accidental hybrids.

\section{A coalescent-based ABC analysis of the gibbon phylogeny}

Unless species branch lengths are several orders of magnitude larger than the expected time to the most recent common ancestor of sequences within a species, it is important to model stochasticity in the distribution of gene trees across loci when 
Table 1 Gibbon samples undergoing second generation sequencing

\begin{tabular}{|c|c|c|c|c|c|c|c|}
\hline Chr no. & Genus & Species & Common name & Code & Sex & Origin & Mean coverage \\
\hline \multirow[t]{2}{*}{52} & Nomascus & Nomascus leucogenys & Northern white-cheeked & NLE & M & Parents WB & 13.78 \\
\hline & & & & & $\mathrm{F}$ & Parents WB & 11.50 \\
\hline \multirow[t]{2}{*}{50} & Symphalangus & Symphalangus syndactylus & Siamang & SSY & $M$ & Sire $W B$, dam $C B$ & 12.80 \\
\hline & & & & & $\mathrm{F}$ & parents $C B$ & 19.53 \\
\hline \multirow[t]{2}{*}{38} & Hoolock & Hoolock leuconedys & Eastern hoolock gibbon & HLE & M & WB & 19.15 \\
\hline & & & & & $\mathrm{F}$ & WB & 14.36 \\
\hline 44 & Hylobates & Hylobates pileatus & Pileated gibbon & $\mathrm{HPI}$ & M & Parents WB & 14.33 \\
\hline 44 & Hylobates & Hylobates moloch & Javan gibbon & $\mathrm{HMO}$ & $\mathrm{F}$ & Sire $W B$, dam $C B$ & 12.96 \\
\hline
\end{tabular}

WB, wild born; $C B$, born in captivity.

inferring an underlying species tree (Rosenberg and Nordborg 2002). Current Bayesian coalescent-based methods such as BEAST (Drummond and Rambaut 2007) that explicitly take into account sequence and population divergence simultaneously to infer species trees are generally computationally intractable for large datasets (Bryant et al. 2012). Therefore, to infer the species topology for gibbon genera we developed an $\mathrm{ABC}$ (Beaumont et al. 2002) method for inference of a species tree with four taxa. The method can also infer species divergence times and effective population sizes for a given topology, can handle large amounts of sequence data, is not dependent on haplotype phase, and incorporates information derived from our modeling of errors from comparing WGS with high coverage WES data.

Analogous to the Bayesian approach of Gronau et al. (2011), which uses an analytical derivation to determine the likelihood of the full data given typical population genetic parameters, the data required for this $\mathrm{ABC}$ method are short, independent loci as we assume no intralocus recombination and free recombination between loci. The latter is a necessary convenience given that no recombination map is currently available for gibbons. Thus, we assembled a set of independent "nongenic" sequences that mapped at least $50 \mathrm{~kb}$ away from genes ( 12,000 1-kb loci) and that excluded CpG consistent sites as well as evolutionarily conserved elements (Siepel et al. 2005) (Figure S1). Mutations detected in these loci are expected to represent neutral variation and to evolve at a relatively constant rate. To reduce reference-mapping bias, we also assembled an analogous set of independent "genic" loci that span exons ( 11,000 200-bp loci) and that should have lower diversity, recognizing that these loci may have been subjected to natural selection, which may bias any parameter estimates.

Analysis of pseudo-observed data generated by simulations demonstrated that we were able to detect the correct topology from randomly drawn datasets using our method $88.4 \%$ of the time, with the correct model among the three highest posterior probabilities $99 \%$ of the time (Figure S4). Analysis of a more targeted set of pseudo-observed data demonstrated that the method is only likely to fail when an internal branch is extremely small (almost instantaneous in evolutionary terms) or when the total height of the tree in units of expected number of substitutions is on the order of 0.001 (equivalent to $\sim 1$ million years for apes) (Figure S5), which is unrealistic for gibbons.
As most $\mathrm{ABC}$ analyses are based on performing simulations to approximate an otherwise analytically intractable likelihood function, we also attempted to stochastically model sequence errors (missing singletons and incorrect genotype calls at other segregating sites) that are likely to have occurred in the real second generation sequencing data. Errors were introduced into coalescent simulations by an $E$ model constructed by comparing the WGS with the high coverage WES data. By incorporating this $E$ model we found through simulated pseudo-observed data that we could infer more accurate estimates of $\theta$ and $\tau$ under very simple demographic scenarios (one population with a constant size $\theta$ through time and two populations of constant size that diverged at some time, $\tau$, in the past) (Figure S6). A full description of the above validation of our $\mathrm{ABC}$ framework using pseudo-observed data are given in Supporting Information.

Prior to the $A B C$ analysis of the real data we examined the one-dimensional distribution for each individual summary statistic from 10,000 random simulations from the $\theta$ and $\tau$ parameter space and found a good fit to our nongenic and genic observed data, while a PCA also demonstrated a good multidimensional fit (Figure S7).

Table 2 shows the posterior probabilities from the ABC analysis for all phylogenetic models for the observed data for both the nongenic and genic loci using the corrected (with stochastic errors introduced via the $E$ model) and uncorrected coalescent simulations (a total of four analyses). No topology dominates the analysis, with three to four topologies having posterior probabilities $>10 \%$ in the corrected simulations. The best topology using nongenic and genic loci for the corrected simulations differ, and both still maintain relatively low posterior probabilities of $\leq 19 \%$. Two topologies appear most prominent with posterior probabilities $>10 \%$ in all four analyses and the highest means across all four analyses and both (genic and nongenic) corrected analyses. One is the most frequently observed topology in the sequence divergence analysis (((SSY, HLE), NLE),(HPI, HMO)) of Carbone et al. (2014) and the other is a related topology where (HPI, HMO) and NLE are swapped as the most external groups with HLE and SSY remaining as sister taxa. Together the posterior probability for both these related topologies sum to $30-32 \%$. However, in general the posterior probabilities are lower than typically observed in our pseudo-observed datasets, suggesting that we have little confidence in the true topology. This is consistent 
Table 2 Posterior probabilities for the 15 possible four-population topologies for nongenic and genic loci

\begin{tabular}{|c|c|c|c|c|}
\hline \multirow[b]{2}{*}{ Topology } & \multicolumn{2}{|c|}{ Nongenic } & \multicolumn{2}{|c|}{ Genic } \\
\hline & Corrected & Uncorrected & Corrected & Uncorrected \\
\hline (((SSY,HLE)NLE)(HPI,HMO)) & 0.16 & 0.15 & 0.19 & 0.15 \\
\hline$((((\mathrm{HPI}, \mathrm{HMO}) \mathrm{NLE}) \mathrm{SSY}) \mathrm{HLE})$ & 0.19 & 0.14 & 0.11 & 0.08 \\
\hline$(((\mathrm{SSY}, \mathrm{HLE})(\mathrm{HPI}, \mathrm{HMO})) \mathrm{NLE})$ & 0.14 & 0.23 & 0.13 & 0.19 \\
\hline ((((HPI,HMO)NLE)HLE)SSY) & 0.13 & 0.11 & 0.06 & 0.05 \\
\hline$(((\mathrm{NLE}, \mathrm{HLE}) \mathrm{SSY})(\mathrm{HPI}, \mathrm{HMO}))$ & 0.06 & 0.05 & 0.10 & 0.08 \\
\hline ((((HPI,HMO)SSY)NLE)HLE) & 0.07 & 0.06 & 0.08 & 0.07 \\
\hline ((((HPI,HMO)SSY)HLE)NLE) & 0.05 & 0.07 & 0.07 & 0.14 \\
\hline$(((\mathrm{HPI}, \mathrm{HMO}) \mathrm{NLE})(\mathrm{SSY}, \mathrm{HLE}))$ & 0.05 & 0.04 & 0.05 & 0.03 \\
\hline$(((\mathrm{NLE}, \mathrm{SSY}) \mathrm{HLE})(\mathrm{HPI}, \mathrm{HMO}))$ & 0.03 & 0.03 & 0.06 & 0.04 \\
\hline (((NLE,HLE)(HPI,HMO))SSY) & 0.04 & 0.03 & 0.04 & 0.04 \\
\hline (((NLE,SSY)(HPI,HMO))HLE) & 0.03 & 0.03 & 0.03 & 0.02 \\
\hline ((((HPI,HMO)HLE)SSY)NLE) & 0.02 & 0.04 & 0.03 & 0.06 \\
\hline ((((HPI,HMO)HLE)NLE)SSY) & 0.02 & 0.02 & 0.02 & 0.02 \\
\hline$(((\mathrm{HPI}, \mathrm{HMO}) \mathrm{SSY})(\mathrm{NLE}, \mathrm{HLE}))$ & 0.01 & 0.01 & 0.03 & 0.02 \\
\hline$(((\mathrm{HPI}, \mathrm{HMO}) \mathrm{HLE})(\mathrm{NLE}, \mathrm{SSY}))$ & 0.01 & 0.01 & 0.01 & 0.00 \\
\hline
\end{tabular}

Boldface type indicates the topology identified using sequence divergence in Carbone et al. (2014).

with the hypothesis of a rapid radiation of gibbon species from a large ancestral population.

The simplest phylogenetic description of this process would be a four-way hard polytomy. Therefore we constructed an additional model with all four genera diverging at the same time and analyzed this scenario within the same $A B C$ framework as the previously examined 15 bifurcating topologies (i.e., we examined 16 different models in total). This did not affect our ability to infer the correct model using pseudo-observed datasets. As with considering only strictly bifurcating topologies, we were able to detect the correct topology from randomly drawn datasets from all 16 models $87.3 \%$ of the time. Of the 16 individual models, the instantaneous model was the one with the lowest proportion of correctly predicted pseudo-observed datasets but was still high at $82.4 \%$.

Despite having the lowest predictive value, when we examined the real data the posterior probability for the instantaneous model ranged from $87-90 \%$ for both the nongenic and genic loci and for the corrected and uncorrected simulations (Table 3). The posterior probabilities of the 15 bifurcating topologies after the addition of the instantaneous model were necessarily much lower but still highly correlated with the previous values with $r^{2}$ ranging between 0.91 and 0.98 . Thus, our $A B C$ analysis strongly supports a relatively instantaneous hard polytomy for the divergence of the four gibbon genera over that of a particular bifurcating topology. Evidence for this can also be seen visually by examining a PCA of the summary statistics for 1000 random datasets from each of the 16 models, with the instantaneous model lying within the center of the cloud of all models and the observed data found firmly within this part of the cloud (Figure S8). Other polytomy combinations may also fit the data (for example a model with the initial divergence of three lineages, followed by a later Hoolock and Symphalangus divergence) but our ability to reliably discriminate such additional intermediate models is likely to further worsen given our instantaneous model already shows reduced predictive ability compared to the other fully bifurcating models.

\section{Estimation of parameters describing gibbon demography}

To estimate when this rapid radiation may have taken place, we constructed a model where all four genera diverge simultaneously with the addition of a subsequent divergence of the two Hylobates species. This resulted in a model with seven $\theta$ and two $\tau$ parameters. The summary statistics from the nongenic loci were transformed into PLS components to infer these parameters. Parameter estimates and posterior distributions are shown in Table S3 and Figure S9. These results are based on 15 PLS components, the value at which the largest reduction in the RMSE was observed across all parameters, Figure S10, and for which the C.I. values for $\tau$ were considered relatively reliable based on how often the true value fell within the estimated 95\% C.I. using 1000 pseudo-observed datasets (Veeramah et al. 2012).

Observed values of $\pi$ described above were within the $95 \%$ C.I. for the $\theta$ values estimated by the $A B C$ analysis for present-day species and showed the same relative pattern with the highest value in the NLE and lowest value in the HPI sample. The divergence time, $\tau_{1}$, for the two Hylobates samples was $\sim 50 \%$ less than that for the divergence time of the four gibbon genera, $\tau_{2}$, which is consistent with the relative difference in sequence divergence of $\sim 50 \%$ seen in Carbone et al. (2014). Because the priors were $\log _{10}$ scaled, the associated $95 \%$ C.I. values potentially could be larger in absolute values (i.e., $10^{\wedge} \mathrm{val}$ ) than if the observed posterior distribution had been shifted toward a smaller branch length. Therefore, we reran the $\mathrm{ABC}$ analysis using unscaled flat priors for the two $\tau$ values, which resulted in highly similar median values but much narrower 95\% C.I.'s (Table 4, Table S4, Figure S11). We note that these C.I.'s were somewhat anticonservative as assessed by pseudo-observed datasets (see column "HDPI 95\% fit" of Table 4 and Table S4). When we assume a $\mu$ of 
Table 3 Posterior probabilities for the 15 possible four-population topologies as well as a instantaneous four-way hard polytomy for nongenic and genic loci

\begin{tabular}{|c|c|c|c|c|}
\hline \multirow[b]{2}{*}{ Topology } & \multicolumn{2}{|c|}{ Nongenic } & \multicolumn{2}{|c|}{ Genic } \\
\hline & Corrected & Uncorrected & Corrected & Uncorrected \\
\hline Instant & 0.874 & 0.859 & 0.902 & 0.899 \\
\hline (((SSY,HLE)NLE)(HPI,HMO)) & 0.024 & 0.023 & 0.018 & 0.017 \\
\hline$((((\mathrm{HPI}, \mathrm{HMO}) \mathrm{NLE}) \mathrm{SS} Y) \mathrm{HLE})$ & 0.024 & 0.023 & 0.011 & 0.011 \\
\hline$(((\mathrm{SSY}, \mathrm{HLE})(\mathrm{HPI}, \mathrm{HMO})) \mathrm{NLE})$ & 0.023 & 0.037 & 0.016 & 0.018 \\
\hline$((((\mathrm{HPI}, \mathrm{HMO}) \mathrm{NLE}) \mathrm{HLE}) \mathrm{SSY})$ & 0.015 & 0.016 & 0.007 & 0.008 \\
\hline ((((HPI,HMO)SSY)NLE)HLE) & 0.008 & 0.007 & 0.010 & 0.007 \\
\hline$(((\mathrm{HPI}, \mathrm{HMO}) \mathrm{NLE})(\mathrm{SSY}, \mathrm{HLE}))$ & 0.007 & 0.006 & 0.005 & 0.002 \\
\hline$(((\mathrm{NLE}, \mathrm{HLE}) \mathrm{SSY})(\mathrm{HPI}, \mathrm{HMO}))$ & 0.007 & 0.007 & 0.009 & 0.008 \\
\hline ((((HPI,HMO)SSY)HLE)NLE) & 0.005 & 0.008 & 0.007 & 0.013 \\
\hline$(((\mathrm{NLE}, \mathrm{HLE})(\mathrm{HPI}, \mathrm{HMO})) \mathrm{SSY})$ & 0.003 & 0.003 & 0.004 & 0.005 \\
\hline$(((\mathrm{NLE}, \mathrm{SSY}) \mathrm{HLE})(\mathrm{HPI}, \mathrm{HMO}))$ & 0.002 & 0.002 & 0.002 & 0.003 \\
\hline ((((HPI,HMO)HLE)NLE)SSY) & 0.002 & 0.002 & 0.002 & 0.002 \\
\hline$(((\mathrm{NLE}, \mathrm{SSY})(\mathrm{HPI}, \mathrm{HMO})) \mathrm{HLE})$ & 0.002 & 0.002 & 0.001 & 0.001 \\
\hline ((((HPI,HMO)HLE)SSY)NLE) & 0.002 & 0.004 & 0.002 & 0.003 \\
\hline$(((\mathrm{HPI}, \mathrm{HMO}) \mathrm{SSY})(\mathrm{NLE}, \mathrm{HLE}))$ & 0.001 & 0.001 & 0.003 & 0.002 \\
\hline$(((\mathrm{HPI}, \mathrm{HMO}) \mathrm{HLE})(\mathrm{NLE}, \mathrm{SSY}))$ & 0.001 & 0.000 & 0.000 & 0.000 \\
\hline
\end{tabular}

$1 \times 10^{-9}$ per site per year *3/4 (to take into account that we excluded CpG sites) (Hodgkinson and Eyre-Walker 2011) this results in an estimate for the time of the gibbon radiation of $1.6+3.5=5.1$ MYA $\left(\tau_{1}-\tau_{2}\right.$ combined limits of $95 \%$ C.I. $2.5-$ 7.7 MYA) and a split time of 1.6 MYA (95\% C.I. 0.6-2.9 MYA) for the two Hylobates samples. In addition, assuming 10 years per generation for gibbons (Harvey et al. 1987) and thus a $\mu$ of $7.5 \times 10^{-9}$ per generation, $N_{\mathrm{e}}$ for extant species varies from 57,000 (NLE) to 7500 (HPI). Interestingly, the ancestral gibbon $N_{\mathrm{e}}$ is estimated to be much larger at 132,000 (107,000-162,000) (Figure 1A) as would be expected if substantial ILS was observed. It should be noted that the estimate of the ancestral Hylobates population size (based on $\theta_{\mathrm{T} 1}$ ) may be somewhat unreliable as the regressed posterior distribution shows a major shift from the raw retained posterior distribution (Figure S11) while this was also the $\theta$ value for which the largest number of PLS components was needed to obtain a reasonable reduction in the RMSE (Figure S12).

One potential source of error in estimating parameters is ancestral state misidentification due to back mutations along the human lineage, which was used as an outgroup (Hernandez et al. 2007). Our simulated data assumed an infinites sites model. Assuming a human-gibbon split time of 16.8 MYA and $\mu$ of $1 \times 10^{-9}$ per site per year, each site has $\sim 98 \%$ chance $\left[\left(1-1 \times 10^{-9}\right)^{\wedge} 16,800,000\right]$ of not experiencing a substitution along the human branch. Therefore, we conducted the $\mathrm{ABC}$ parameter estimation on a set of $10^{5}$ simulations where we incorporated a $2 \%$ rate of random ancestral allele misidentification. Though this binary model of back mutation is highly simplistic (e.g., it does not take into account mutations to another base-pair type or trinucleotide context), we found it had only minimal impact on our $95 \%$ C.I.'s compared with the same number of simulations that did not incorporate some ancestral state misidentification error (Table S5). This suggests that our divergence time estimates may be only slightly underestimated by not accounting for this error.

To investigate the effect of imposing a model of instantaneous speciation rather than bifurcating species divergence on our parameter inference, we also modeled the five gibbon species assuming the best sequence phylogeny from Carbone et al. (2014) and that was also suggested by our ABC model choice analysis, [(((SSY, HLE)NLE)(HPI,HMO))] (Table S6, Figure 1B, Figure S13, Figure S14). The median estimates of the posterior distributions for the seven $\theta$ parameters common to both the bifurcating and instantaneous models (five extant population values as well $\theta_{\mathrm{T} 1}$ and $\theta_{\mathrm{anc}}$ ) were similar, while the $95 \%$ C.I. for $\theta_{\mathrm{T} 1}$ and $\theta_{\mathrm{T} 2}$ were broad and uninformative. Consistent with the rapid speciation hypothesis (even when allowing bifurcating speciation), $\tau_{2+} \tau_{3}+\tau_{4}$ was roughly equivalent to $\tau_{2}$ for the instantaneous speciation model, with $\tau_{3}$ and $\tau_{4}$ being an order of magnitude smaller (i.e., very short internal branch lengths).

Table 4 Posterior estimates for an instantaneous speciation model for gibbon genera using a flat prior for $\tau$

\begin{tabular}{|c|c|c|c|c|c|}
\hline \multirow[b]{3}{*}{ Parameter } & \multirow[b]{3}{*}{ HDPI $95 \%$ fit $^{a}$} & \multicolumn{4}{|c|}{ Posterior estimation $b$} \\
\hline & & \multirow[b]{2}{*}{ Mode } & \multirow[b]{2}{*}{ Median } & \multicolumn{2}{|c|}{ HDPI 95} \\
\hline & & & & Lower & Upper \\
\hline$\theta_{\mathrm{NLE}}$ & 0.930 & $1.71 \mathrm{E}-03$ & $1.72 \mathrm{E}-03$ & 1.07E-03 & $2.73 \mathrm{E}-03$ \\
\hline$\theta_{\mathrm{SSY}}$ & 0.936 & $9.25 \mathrm{E}-04$ & $9.24 \mathrm{E}-04$ & 5.97E-04 & $1.43 \mathrm{E}-03$ \\
\hline$\theta_{\mathrm{HLE}}$ & 0.937 & 4.17E-04 & 4.17E-04 & $2.63 \mathrm{E}-04$ & $6.58 \mathrm{E}-04$ \\
\hline$\theta_{\mathrm{HPI}}$ & 0.968 & $2.24 \mathrm{E}-04$ & $2.25 \mathrm{E}-04$ & 1.30E-04 & $3.92 \mathrm{E}-04$ \\
\hline$\theta_{\mathrm{HMO}}$ & 0.974 & 8.29E-04 & $8.32 \mathrm{E}-04$ & $4.13 \mathrm{E}-04$ & $1.68 \mathrm{E}-03$ \\
\hline$\theta_{\mathrm{T} 1}$ & 0.958 & $3.54 \mathrm{E}-03$ & $3.80 \mathrm{E}-03$ & 7.69E-04 & $1.90 \mathrm{E}-02$ \\
\hline$\theta_{\text {Tanc }}$ & 0.964 & 3.97E-03 & $3.97 \mathrm{E}-03$ & $3.23 \mathrm{E}-03$ & $4.86 \mathrm{E}-03$ \\
\hline$\tau_{1}$ & 0.905 & 1.05E-03 & $1.23 \mathrm{E}-03$ & $5.01 \mathrm{E}-04$ & $2.18 \mathrm{E}-03$ \\
\hline$\tau_{2}$ & 0.911 & $2.69 \mathrm{E}-03$ & $2.59 \mathrm{E}-03$ & $1.41 \mathrm{E}-03$ & $3.63 \mathrm{E}-03$ \\
\hline
\end{tabular}


A

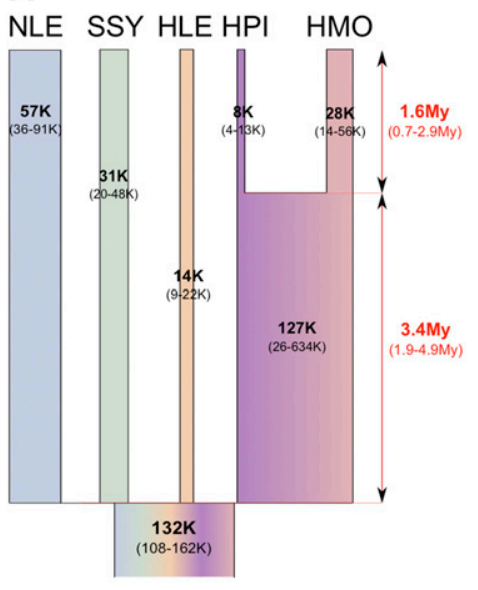

$B$

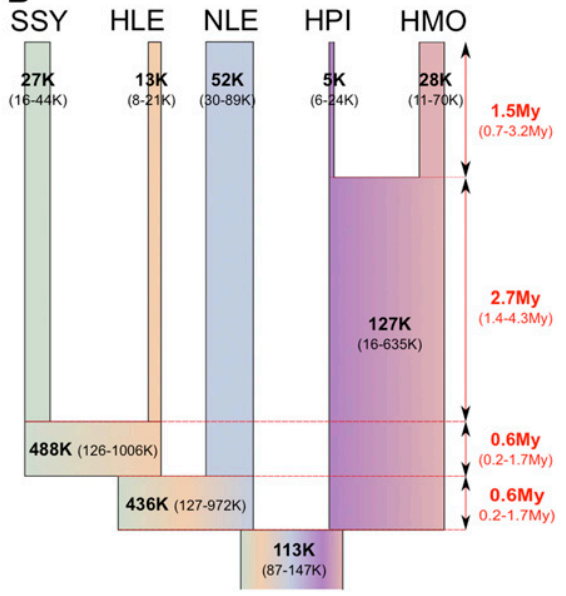

Figure 1 Parameter estimates for the instantaneous radiation (A) and bifurcating speciation (B) model for gibbon genera. $\mu=7.5 \times 10^{-9} /$ site/generation, 10 years per generation. $\theta_{\mathrm{T} 2}-$ and $\theta_{\mathrm{T} 3}$-based $N_{\mathrm{e}}$ values are not to scale.
We also applied the $1 \mathrm{~kb}$ data to the method of Gronau et al. (2011) as this approach is based on a similar model (i.e., the coalescent with population divergence) as our bifurcating $\mathrm{ABC}$ analysis; however, it should be more accurate for parameter estimation as it is based on an exact model likelihood rather than an approximation (although it does not currently incorporate the possibility of sequence error). While the implementation for estimating divergence times is slightly different (e.g., our $\mathrm{ABC}$ approach uses time intervals between divergence events rather than absolute divergence times from the present), the results are very similar: very short internal branch lengths among gibbon genera and a total gibbon genera divergence time of 5-6 MYA. However, as expected when using the full data rather than an approximation of it (Csilléry et al. 2010), the 95\% C.I.'s estimated by G-PhoCS (Table S1) were substantially narrower than those estimated by $\mathrm{ABC}$, which are likely inflated because of a loss of information through the use of insufficient summary statistics.

\section{Allele sharing and D-statistic analysis}

Because of the small sample sizes and large divergence times, it is not expected that we would have the ability to infer gene flow if added as an additional parameter (whether an instantaneous pulse or continuous migration after divergence) in our $\mathrm{ABC}$ analysis. Although intergenera hybrids have been observed in captivity, they are almost certainly infertile as a result of the complicated patterns of homology that would disrupt meiotic pairing. Moreover, such matings have never been observed in the wild, even for sympatric species (Hirai et al. 2007). Therefore, it is unlikely that gene flow would continue for long after divergence as is typically modeled using isolation with migration approaches. Of course, this assumption depends on the rate of karyotypic change, which is thought to have occurred relatively soon after divergence and to have contributed to the speciation process (Carbone et al. 2014). Thus, accounting for biologically meaningful gene flow would increase the complexity of the model beyond what can likely be reliably inferred using $\mathrm{ABC}$ for this dataset.
However, a fairly simply measure that can help to infer admixture events (although not necessarily help to reveal the mode, timing, or extent of admixture) is the $D$-statistic (Durand et al. 2011). We first examined patterns of allele sharing across the whole genome by tallying the state of each genus at variable sites by (a) choosing sites that met certain quality criteria (as determined by our masks) and that were homozygous for the same allele in both individuals from a genus (filt1), (b) randomly sampling one allele from the two genotypes from a genus for sites that met the same quality criteria as a (filt2), or (c) randomly sampling one read from both individuals in a genus at a site (filt3) (Table S7a). We also repeated this at the species level, using only the highest coverage sample from each species (in this case filt1 reflects homozygous allele sharing) (Table S7c). Results were not qualitatively different using these different filtering criteria.

Consistent with our ABC analysis and Wall et al. (2013), SSY and HLE share the largest number of alleles. Interestingly, while NLE and the two Hylobates samples share a fairly low number of alleles compared with other pairwise comparisons, they both share more alleles with SSY than HLE. We performed a $D$-statistic analysis that demonstrated this excess sharing was statistically significant (Table S7, b and d). Under the assumption that SSY and HLE diverged last among the four genera as indicated in our $\mathrm{ABC}$ analysis, such a pattern is consistent with a model involving two independent gene flow events into SSY from both NLE and Hylobates after they diverged from HLE. An alternative model that does not invoke postdivergence gene flow involves the maintenance of longterm population structure between the ancestors of HLE and the ancestral population giving rise to the other gibbon genera (Figure 2). We attempted to incorporate population structure into our $\mathrm{ABC}$ framework but found via simulations that we could not distinguish between these models, especially given a parameter space consisting of short internal branch lengths as observed in this dataset (data not shown).

We also used the $D$-statistic to examine whether there was any evidence of unbalanced allele sharing between the two Hylobates species. While the $D$-statistic slightly favored 
more allele sharing between HMO and the other three genera, the values were generally quite low and the $Z$-scores were only greater than $|2|$ under filtering scheme 2 .

\section{Discussion}

Previous attempts to resolve the phylogenetic relationships among the four gibbon genera based on different genetic systems (karyotypes changes, mtDNA, the Y chromosome, and short autosomal sequences, and ALU repeats) resulted in widely discordant phylogenies. All samples utilized in this study were also analyzed as part of the Gibbon Genome Project (Carbone et al. 2014) where the best supported overall consensus tree based on genome-wide sequence divergence was found to be (((SSY, HLE), NLE),(HPI, HMO)). However, all four gibbon genera demonstrated a narrow range for sequence divergence (1.08-1.12\%; mean $1.10 \%)$. Here, we developed a potentially powerful species tree analysis framework for four taxa that made use of genome-wide second generation sequencing data and took into account discordant gene trees and applied them to the problem of the phylogenetic relationships of the four gibbon genera. Despite the availability of whole genome sequence data and the methodology demonstrating success with most simulated pseudo-observed datasets, we could not confidently resolve the phylogenetic relationships between Nomascus, Symphalangus, Hylobates, and Hoolock, although Symphalangus and Hoolock may represent the most recently diverged genera. This latter result is consistent with the best consensus gene tree identified by Carbone et al. (2014) and Wall et al. (2013).

The most well-supported bifurcating phylogeny is characterized by long external branch lengths and very short internal branch lengths, pointing to a rapid radiation of the four gibbon genera from a large ancestral effective population of $\sim 10^{5}$ individuals. Indeed, when we included an additional model representing a four-way hard polytomy in our $\mathrm{ABC}$ analyses we found substantial support for this scenario of instantaneous divergence over any of the individual bifurcating topologies (at least at the level of resolution of branch lengths afforded by the data). This demographic scenario would explain previous observations of genome-wide ILS (Wall et al. 2013) and discordant phylogenies across smaller datasets. However, we note that an alternative explanation is that the ancestral gibbon population already exhibited structure prior to the divergence of the four gibbon genera.

It is possible that such a stark restructuring of the gibbon population during this proposed radiation event was driven by some major climatic or geological shift. This is particularly likely as gibbons reside predominantly on the relatively shallow Sunda continental shelf of Southeast Asia. At various times, sea level changes and volcanic activity significantly altered the amount of habitable land (i.e., above sea level) in this region. As gibbons live a highly arboreal lifestyle, any reduction or fragmentation of their native forest habitats could have led to extreme genetic isolation between geographically dispersed populations. This, coupled with a rapid evolution of

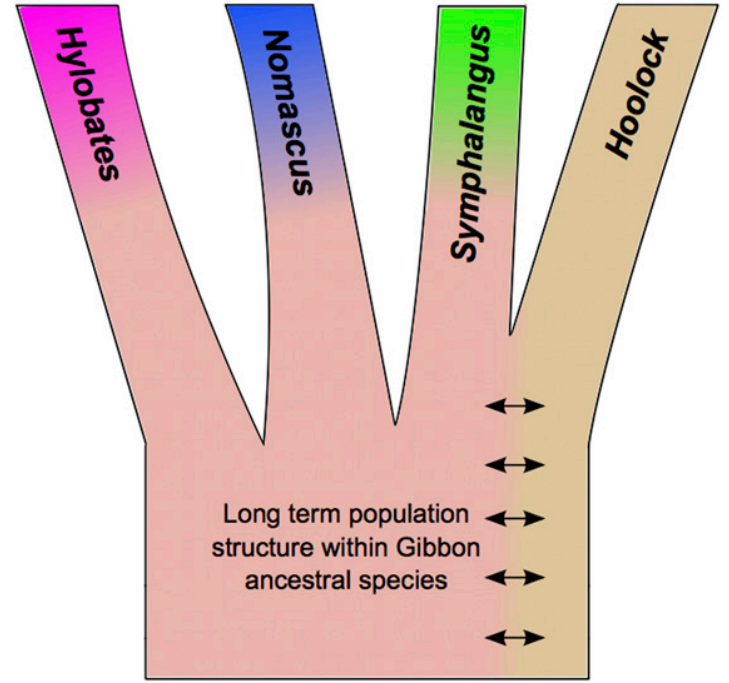

Figure 2 Cartoon of proposed model of ancestral population structure among gibbon genera.

karyotype differences, could have driven the speciation process among these gibbon taxa.

\section{Uncertainty in timing of the gibbon radiation}

It is important to note that associating the timing of speciation with the geological or climatological record is complicated by uncertainty in how we calibrate our estimates of $\tau$ (i.e., our choice of mutation rate). A phylogenetic estimate of $\mu$ for great apes that is often used is $\sim 1 \times 10^{-9}$ per site per year, an estimate based on calibrating sequence divergence with the fossil record (Takahata and Satta 1997; Nachman and Crowell 2000). This would place the radiation of gibbon genera within the early Pliocene $\sim 5$ MYA. Interestingly, it has been proposed that the Sunda shelf was largely one land mass up to 5 MYA (Outlaw and Voelker 2008), after which sea levels began to rise until $\sim 3$ MYA (Cichon et al. 2004) leading to the fragmenting of the region. There is evidence for an increased rate of divergence in other plants and animals during this early Pliocene window (Gorog et al. 2004; Outlaw and Voelker 2008; Akula et al. 2010; López-Guillermo et al. 2010) and thus, it is possible that gibbon divergence may have been driven by the same process.

On the other hand, a value of $\mu=0.5 \times 10^{-9}$ per site per year has recently been estimated using direct observation of mutations in human trios and quartets (Roach et al. 2010; Kong et al. 2012). Scally and Durbin (2012) attempted to reconcile the phylogenetic and direct pedigree estimates with the fossil record (which itself is used to calibrate the phylogenetic estimate) by invoking the hominid slowdown hypothesis. Under this hypothesis, the increased body size of great apes correlates with a decrease in generation time and a reduction in the annual mutation rate after their divergence from Old World monkeys. Evidence for this comes from evolutionary comparisons of Great Apes to Old World monkeys (e.g., humans have a $30 \%$ slower evolutionary rate as compared to baboons) (Kim et al. 2006). While generally bigger 
than Old World monkeys, the largest gibbons, from the genus Symphalangus, are approximately half the size of the smallest great ape, Pan paniscus. Thus, given that gibbons have smaller body sizes (and shorter generation times) than other apes, it is not clear to what extent the hominid slowdown hypothesis would apply.

Decreasing the mutation rate would lead to a Late Miocene speciation time of up to $\sim 10$ MYA, thus encompassing previous estimates of divergence at 6-8 MYA based on mtDNA (Chan et al. 2010; Matsudaira and Ishida 2010; Van Ngoc et al. 2010). However, fossil calibration-based estimates such as used in these studies are subject to their own biases (Lukoschek et al. 2012), while estimates of demography from a single locus (especially a nonrecombining region of the genome, no matter how well resolved the gene tree) are subject to large evolutionary stochasticity (Rosenberg and Nordborg 2002). It is noteworthy that the $\mathrm{Y}$ chromosome estimate differs from the mtDNA estimate substantially (5 and 9 MYA, respectively) despite application of the same calibration procedures (Chan et al. 2012).

Our results do appear to rule out the hypothesis of Chivers (1977), which suggests a Late Pleistocene divergence of gibbon genera. Despite this, the constant formation and destruction of land bridges during the Pleistocene that drives the Pleistocene pump hypothesis (Gorog et al. 2004; Akula et al. 2010) may have contributed to divergence of the several species within each gibbon genus (for example the pileatus/moloch split we observe $\sim 1.6 \mathrm{MYA}$ ). Though the exact numbers are the subject of some debate, it is generally accepted that there are at least 7, 6, and 2 different Hylobates, Nomascus, and Hoolock species, respectively (Van Ngoc et al. 2010; Mittermeier et al. 2013; Rowe and Myers 2015). Movement during these periods likely explains the current distribution of Hylobates species both on the mainland and the islands of Sumatra, Borneo, and Java, especially when one considers that gibbons probably cannot swim. Today neighboring gibbon species are largely isolated from each other by rivers. Further whole genome sequencing of multiple individuals from additional species, along with the application of powerful genomic methods to infer gene flow or admixture between species, will provide invaluable information for inferring the relationships among gibbon species across Southeast Asia. In addition, while it is well recognized that land bridges certainly formed during the Pleistocene, there is still great uncertainty as to whether these would have involved forest canopy or more savannah-like vegetation (Bird et al. 2005). Analysis of patterns of historic gene flow among the tree dwelling gibbons may help shed light on this process. Recent work using small amounts of autosomal sequence data ( $\sim 11 \mathrm{~kb}$ ) has already found evidence of asymmetrical gene flow between Hylobates species currently located on different islands (Chan et al. 2013) while a basic $D$-statistic analysis in this article also hinted at the possibility of introgression between genera after divergence.

Challenges in the use of whole genome sequence data for estimating demographic parameters: Despite the fact that we generated whole genome sequences, it is important to appreciate that the explicit $\mathrm{ABC}$ modeling performed here utilized only a small amount of the total available data. A pairwise sequentially Markovian coalescent (PSMC) (Li and Durbin 2011) analysis presented in Carbone et al. (2014) takes a different approach to utilizing genome-scale sequence data. By incorporating patterns of genetic diversity across individual genome sequences, important insights can be gained into changing $N_{\mathrm{e}}$ through time. To summarize these findings in the context the $\mathrm{ABC}$ demographic analysis presented here, both the NLE and HMO populations show major fluctuations in effective population size during the time frame after gibbon genera diverged, when Pleistocene geological and climate shifts were taking place.

Composite likelihood methods that evaluate the entire allele frequency spectrum (AFS) across many populations may also prove useful for inference in situations such as the one presented here (Gutenkunst et al. 2009). These methods are likely to be particularly effective for estimating additional parameters such as recent size changes and migration when many individuals are sampled from multiple species compared to our approach, which uses only a summary of the AFS, though demographic events on the order of millions of years ago may still not be tractable regardless of sample size (Robinson et al. 2014). These approaches can also take advantage of recent methods that allow relatively unbiased estimation of the AFS from even low coverage second generation sequencing data (though this currently must be done for each population separately). There have also been advances in extending these AFSbased approaches to many (more than three) populations and testing alternative scenarios even when using nonnested models and composite rather than full likelihoods (Excoffier et al. 2013), though whether these can reliably be used for contrasting many different models simultaneously in a phylogenetic context has yet to be explored. One notable limitation of these approaches is that they assume independence of sites and ignore linkage, and thus inference of migration and admixture is potentially underpowered (Sousa and Hey 2013).

However, to fully exploit whole genome data for demographic inference using coalescent methods, it will be vital to construct genetic maps in gibbons, preferably separately for each genus, such that recombination can be appropriately incorporated into any population genetic analysis. In addition, despite applying a correction factor in our analysis, reference bias toward Nomascus genomes was evident in our data, and it is likely that even more reference bias exists than we actually observe due to the variable karyotypes across genera. It seems unlikely that further large-scale Sanger sequencing will be used to link up scaffolds or generate reference genomes for the other three non-Nomascus genera, while short-read Illumina data are not suited for this task. However, the application of new sequencing technologies with long reads such as the PacBio (English et al. 2012) and nanopore (Schneider and Dekker 2012) technologies may provide useful and relatively low cost alternatives to assemble more robust reference genomes. This should lead to more powerful demographic and evolutionary analyses of gibbons in the future. 


\section{Using $A B C$ in phylogenetics}

There is currently one published generalized ABC phylogenetic approach (ST-ABC) (Fan and Kubatko 2011). This method relies on having accurately phased sequence as it treats frequencies of gene tree topologies across loci as the data rather than summary statistics, and has only been tested and applied to relatively small datasets. However, it has also been questioned whether ST-ABC can accurately approximate the posterior distribution, as it relies on expectations of the distribution of gene trees rather than random simulations that incorporate sampling variability (Buzbas 2012). Our ABC approach does not have these limitations. While it could not reliably infer a particular bifurcating gibbon genera topology with any confidence because of the extremely short internal branch lengths (as we showed with our additional analysis of an instantaneous speciation model, a bifurcating topology may not actually exist in this case of gibbons), simulations suggest our $\mathrm{ABC}$ approach should allow us to infer the correct species topology for four taxa in most reasonable cases.

However, it is important to appreciate that the framework applied here is tailored for this particular dataset involving unphased genome-wide data from a few individuals per taxa that diverged within the last 10 million years or so. How it would scale up with regard to speed with increasing numbers of samples, and how much accuracy and precision would be lost with fewer loci requires further investigation. It is possible that adding variance in the number of shared sites across loci as a summary statistic may prove useful in this case. In addition, increasing the number of taxa considered (even by one) could prove problematic due to a rapid increase in the parameter space (i.e., a large increase in the number of possible topologies) and an increase in the numbers of summary statistics needed to capture the phylogenetic structure (i.e., the potential impact of the "curse of dimensionality"). Combining more efficient ways of traversing tree space (Bryant et al. 2012) may help with regard to the former issue, while choosing a more efficient set of summary statistics (e.g., via PLS) may improve the latter; however, there are still likely to be limits to how well the data can be summarized in just a few summary statistics for large phylogenies. Another potential issue of our approach that would place limits on the possible time depth for the phylogeny considered is the assumption of an infinite sites mutation model. It would be trivial to incorporate more complex substitution models, although this would also increase the computational burden.

With these improvements in mind, the $\mathrm{ABC}$ family of methods has the potential to provide a useful and flexible phylogenetic tool that balances the need to incorporate large genomic datasets while taking into account gene tree uncertainty and variation in a coalescent framework. Genomic data are being generated at a rapid pace for a diverse set of species and it is clear that phylogenetic methods are required that can accommodate such data. $\mathrm{ABC}$ provides one approach to do this.

\section{Acknowledgments}

We thank Ryan Sprissler and the University of Arizona Genetics Core for assistance with sequencing and Ryan Gutenkunst for computing resources. Support for this work was provided by the National Institutes of Health to J.D.W. and M.F.H. (R01_HG005226) and an European Research Council Starting Grant (260372) and Ministerio de Ciencia e Innovación (Spain) BFU2011-28549 to T.M.-B.

\section{Literature Cited}

Akula, N., M. Cabanero, I. Cardona, and W. Corona, 2010 Speciation dynamics in the SE Asian tropics: Putting a time perspective on the phylogeny and biogeography of Sundaland tree squirrels, Sundasciurus. Mol. Phylogenet. Evol. 55: 711-720.

Beaumont, M. A., W. Zhang, and D. J. Balding, 2002 Approximate Bayesian computation in population genetics. Genetics 162: 2025-2035.

Benson, G., 1999 Tandem repeats finder: a program to analyze DNA sequences. Nucleic Acids Res. 27: 573-580.

Bird, M. I., D. Taylor, and C. Hunt, 2005 Palaeoenvironments of insular Southeast Asia during the Last Glacial Period: a savanna corridor in Sundaland? Quat. Sci. Rev. 24: 2228-2242.

Bryant, D., R. Bouckaert, J. Felsenstein, N. A Rosenberg, and A. RoyChoudhury, 2012 Inferring species trees directly from biallelic genetic markers: bypassing gene trees in a full coalescent analysis. Mol. Biol. Evol. 29: 1917-1932.

Buzbas, E. O., 2012 On the article titled "Estimating species trees using approximate Bayesian computation"(Fan and Kubatko, Molecular Phylogenetics and Evolution 59: 354-363). Mol. Phylogenet. Evol. 65: 1014-1016.

Carbone, L., R. A. Harris, S. Gnerre, and K. R. Veeramah, B. LorenteGaldos et al., 2014 Gibbon genome and the fast karyotype evolution of small apes. Nature 513: 195-201.

Chan, Y.-C., C. Roos, M. Inoue-Murayama, E. Inoue, C.-C. Shih et al., 2010 Mitochondrial genome sequences effectively reveal the phylogeny of Hylobates gibbons. PLoS ONE 5: e14419.

Chan, Y.-C., C. Roos, M. Inoue-Murayama, E. Inoue, C.-C. Shih et al., 2012 A comparative analysis of $\mathrm{Y}$ chromosome and mtDNA phylogenies of the Hylobates gibbons. BMC Evol. Biol. 12: 150

Chan, Y.-C., C. Roos, M. Inoue-Murayama, E. Inoue, C.-C. Shih et al., 2013 Inferring the evolutionary histories of divergences in Hylobates and Nomascus gibbons through multilocus sequence data. BMC Evol. Biol. 13: 82.

Chivers, D. J., 1977 The lesser apes, pp. 539-598 in Primate Conservation, edited by P. R. I. O. Monaco, and H. G. Bourne. Academic Press, New York.

Csilléry K., M. Blum, O. E. Gaggiotti, and O. François, 2010 Approximate Bayesian computation (ABC) in practice. Trends in Ecology \& Evolution. 25: 410-418.

Depristo, M. A., E. Banks, R. Poplin, K. V. Garimella, J. R. Maguire et al., 2011 A framework for variation discovery and genotyping using next-generation DNA sequencing data. Nat. Genet. 43: 491-498.

Drummond, A. J., and A. Rambaut, 2007 BEAST: Bayesian evolutionary analysis by sampling trees. BMC Evol. Biol. 7: 214.

Durand, E. Y., N. Patterson, D. Reich, and M. Slatkin, 2011 Testing for Ancient Admixture between Closely Related Populations. Mol. Biol. Evol. 28: 2239-2252.

English, A. C., S. Richards, Y. Han, M. Wang, V. Vee et al., 2012 Mind the gap: upgrading genomes with Pacific Biosciences RS long-read sequencing technology PLoS ONE 7: e47768. 
Excoffier, L., I. Dupanloup, E. Huerta-Sanchez, V. C. Sousa, and M. Foll, 2013 Robust demographic inference from genomic and SNP data. PLoS Genet 9: e1003905.

Fagundes, N. J. R., N. Ray, M. Beaumont, S. Neuenschwander, F. M. Salzano et al., 2007 Statistical evaluation of alternative models of human evolution. Proc. Natl. Acad. Sci. USA 104: 1761417619.

Fan, H. H., and L. S. Kubatko, 2011 Estimating species trees using approximate Bayesian computation. Mol. Phylogenet. Evol. 59: 354-363.

Fuentes, A., 2000 Hylobatid communities: changing views on pair bonding and social organization in hominoids. Am. J. Phys. Anthropol. 113: 33-60.

Geissmann, T., 2002 Duet-splitting and the evolution of gibbon songs. Biol. Rev. Camb. Philos. Soc. 77: 57-76.

Gorog, A. J., M. H. Sinaga et al., 2004 Vicariance or dispersal? Historical biogeography of three Sunda shelf murine rodents (Maxomys surifer, Leopoldamys sabanus and Maxomys whiteheadi). Biological Journal of the.

Gronau, I., M. J. Hubisz, B. Gulko, C. G. Danko, and A. Siepel, 2011 Bayesian inference of ancient human demography from individual genome sequences. Nat. Genet. 43: 1031-1034.

Gutenkunst, R. N., R. D. Hernandez, S. H. Williamson, and C. D. Bustamante, 2009 Inferring the joint demographic history of multiple populations from multidimensional SNP frequency data. PLoS Genet 5: e1000695.

Hall, M., E. Frank, G. Holmes, B. Pfahringer, P. Reutemann et al., 2008 The WEKA data mining software: an update. ACM SIGKDD Explorations 11: 10-18.

Harvey, P. H., R. D. Martin, and T. H. Clutton-Brock, 1987 Life Histories In Comparative Perspective, University of Chicago Press, Chicago.

Hayashi, S., K. Hayasaka, O. Takenaka, and S. Horai, 1995 Molecular phylogeny of gibbons inferred from mitochondrial DNA sequences: preliminary report. J. Mol. Evol. 41: 359-365.

Hernandez, R. D., S. H. Williamson, and C. D. Bustamante, 2007 Context dependence, ancestral misidentification, and spurious signatures of natural selection. Mol. Biol. Evol. 24: 1792-1800.

Hirai, H., Y. Hirai, H. Domae, and Y. Kirihara, 2007 A most distant intergeneric hybrid offspring (Larcon) of lesser apes, Nomascus leucogenys and Hylobates lar. Hum. Genet. 122: 477-483.

Hodgkinson, A., and A. Eyre-Walker, 2011 Variation in the mutation rate across mammalian genomes. Nat. Rev. Genet. 12: 756766.

Hudson, R. R., 2002 Generating samples under a Wright-Fisher neutral model of genetic variation. Bioinformatics 18: 337-338.

Jin, X., M. He, B. Ferguson, Y. Meng, L. Ouyang et al., 2012 An effort to use human-based exome capture methods to analyze chimpanzee and macaque exomes. PLoS ONE 7: e40637.

Kent, W. J., R. Baertsch, A. Hinrichs, W. Miller, and D. Haussler, 2003 Evolution's cauldron: duplication, deletion, and rearrangement in the mouse and human genomes. Proc. Natl. Acad. Sci. USA 100: 11484-11489.

Kim, S. K., L. Carbone, C. Becquet, A. R. Mootnick, D. J. Li et al., 2011 Patterns of genetic variation within and between gibbon species. Mol. Biol. Evol. 28: 2211-2218.

Kim, S.-H., N. Elango, C. Warden, E. Vigoda, and S. V. Yi, 2006 Heterogeneous genomic molecular clocks in primates. PLoS Genet. 2: e163.

Kong, A., M. L. Frigge, G. Masson, S. Besenbacher, P. Sulem et al., 2012 Rate of de novo mutations and the importance of father's age to disease risk. Nature 488: 471-475.

Leuenberger, C., and D. Wegmann, 2010 Bayesian Computation and Model Selection Without Likelihoods. Genetics 184: 243252.
Li, H., and R. Durbin, 2009 Fast and accurate short read alignment with Burrows-Wheeler transform. Bioinformatics 25: $1754-1760$.

Li, H., and R. Durbin, 2011 Inference of human population history from individual whole-genome sequences. Nature 475: 493496.

López-Guillermo, A., E. Campo, Z. Y. Xue, D. P. Yarnall, J. D. Briley et al., 2010 Elucidating the evolutionary history of the Southeast Asian, holoparasitic, giant-flowered Rafflesiaceae: Pliocene vicariance, morphological convergence and character displacement. Mol. Phylogenet. Evol. 57: 620-633.

Lukoschek, V., J. Scott Keogh, and J. C. Avise, 2012 Evaluating fossil calibrations for dating phylogenies in light of rates of molecular evolution: a comparison of three approaches. Syst. Biol. 61: 22-43.

Lunter, G., and M. Goodson, 2011 Stampy: a statistical algorithm for sensitive and fast mapping of Illumina sequence reads. Genome Res. 21: 936-939.

Matsudaira, K., and T. Ishida, 2010 Phylogenetic relationships and divergence dates of the whole mitochondrial genome sequences among three gibbon genera. Mol. Phylogenet. Evol. 55: 454-459.

McKenna, A., M. Hanna, E. Banks, A. Sivachenko, K. Cibulskis et al., 2010 The Genome Analysis Toolkit: a MapReduce framework for analyzing next-generation DNA sequencing data. Genome Res. 20: 1297-1303.

Meyer, T. J., A. T. McLain, J. M. Oldenburg, C. Faulk, M. G. Bourgeois et al., 2012 An Alu-based phylogeny of gibbons (hylobatidae). Mol. Biol. Evol. 29: 3441-3450.

Mittermeier, R. A., D. E. Wilson, and A. B. Rylands, 2013 Handbook of the Mammals of the World, Lynx Edicions, Barcelona.

Monda, K., R. E. Simmons, P. Kressirer, B. Su, and D. S. Woodruff, 2007 Mitochondrial DNA hypervariable region-1 sequence variation and phylogeny of the concolor gibbons. Nomascus. Am. J. Primatol. 69: 1285-1306.

Mootnick, A. R., 2006 Gibbon (Hylobatidae) species identification recommended for rescue or breeding centers. Primate Conservation 21: 103-138.

Müller, S., M. Hollatz, and J. Wienberg, 2003 Chromosomal phylogeny and evolution of gibbons (Hylobatidae). Hum. Genet. 113: 493-501.

Myers, R. H., and D. A. Shafer, 1979 Hybrid ape offspring of a mating of gibbon and siamang. Science 205: 308-310.

Nachman, M. W., and S. L. Crowell, 2000 Estimate of the mutation rate per nucleotide in humans. Genetics 156: 297-304.

Outlaw, D. C., and G. Voelker, 2008 Pliocene climatic change in insular Southeast Asia as an engine of diversification in Ficedula flycatchers. J. Biogeogr. 35: 739-752.

Patterson, N., A. L. Price, and D. Reich, 2006 Population structure and eigenanalysis. PLoS Genet. 2: e190.

Prado-Martinez, J., P. H. Sudmant, J. M. Kidd, H. Li, J. L. Kelley et al., 2013 Great ape genetic diversity and population history. Nature 499: 471-475.

Rannala, B., and Z. Yang, 2003 Bayes estimation of species divergence times and ancestral population sizes using DNA sequences from multiple loci. Genetics 164: 1645-1656.

Roach, J. C., G. Glusman, A. F. A. Smit, C. D. Huff, R. Hubley et al., 2010 Analysis of genetic inheritance in a family quartet by whole-genome sequencing. Science 328: 636-639.

Robinson J. D., Coffman A. J., Hickerson M. J., Gutenkunst R. N., 2014 Sampling strategies for frequency spectrum-based population genomic inference. BMC Evol. Biol. 14: 254.

Rosenberg, N. A., and M. Nordborg, 2002 Genealogical trees, coalescent theory and the analysis of genetic polymorphisms. Nat. Rev. Genet. 3: 380-390.

Rowe, N., and M. Myers (Editors), 2015 All the World's Primates. Available at: http://alltheworldsprimates.org/Home.aspx. Accessed: 2015. 
Scally, A., and R. Durbin, 2012 Revising the human mutation rate: implications for understanding human evolution. Nat. Rev. Genet. 13: 745-753.

Schneider, G. F., and C. Dekker, 2012 DNA sequencing with nanopores. Nat. Biotechnol. 30: 326-328.

Siepel, A., G. Bejerano, J. S. Pedersen, A. S. Hinrichs, M. Hou et al., 2005 Evolutionarily conserved elements in vertebrate, insect, worm, and yeast genomes. Genome Res. 15: 1034-1050.

Smit A. F. A., Hubley R., Green P., 1996 RepeatMasker Open. Available at: http://www.repeatmasker.org. Accessed: April 25, 2013

Sousa, V. C., and J. Hey, 2013 Understanding the origin of species with genome-scale data: modelling gene flow. Nature Publishing Group 14: 404-414.

Takacs, Z., J. C. Morales, T. Geissmann, and D. J. Melnick, 2005 A complete species-level phylogeny of the Hylobatidae based on mitochondrial ND3-ND4 gene sequences. Mol. Phylogenet. Evol. 36: 456-467.

Takahata, N., and Y. Satta, 1997 Evolution of the primate lineage leading to modern humans: phylogenetic and demographic inferences from DNA sequences. Proc. Natl. Acad. Sci. USA 94: 4811-4815.

Van Ngoc, T., A. R. Mootnick, T. Geissmann, M. Li, T. Ziegler et al., 2010 Mitochondrial evidence for multiple radiations in the evolutionary history of small apes. BMC Evol. Biol. 10: 74
Veeramah, K. R., D. Wegmann, A. Woerner, F. L. Mendez, J. C. Watkins et al., 2012 An early divergence of KhoeSan ancestors from those of other modern humans is supported by an ABCbased analysis of autosomal resequencing data. Mol. Biol. Evol. 29: 617-630.

Wall, J. D., S. K. Kim, F. Luca, L. Carbone, A. R. Mootnick et al., 2013 Incomplete lineage sorting is common in extant gibbon genera. PLOS ONE 8: e53682.

Wegmann, D., C. Leuenberger, and L. Excoffier, 2009 Efficient approximate Bayesian computation coupled with Markov chain Monte Carlo without likelihood. Genetics 182: 1207-1218.

Wegmann, D., C. Leuenberger, S. Neuenschwander, and L. Excoffier, 2010 ABCtoolbox: a versatile toolkit for approximate Bayesian computations. BMC Bioinformatics 11: 116

Whittaker, D. J., J. C. Morales, and D. J. Melnick, 2007 Resolution of the Hylobates phylogeny: Congruence of mitochondrial D-loop sequences with molecular, behavioral, and morphological data sets. Mol. Phylogenet. Evol. 45: 620-628.

Zhong, G., J. Geng, H. K.Wong, Z. Ma, N. Wu, 2004 A semi-quantitative method for the reconstruction of eustatic sea level history from seismic profiles and its application to the southern South China Sea. Earth Planet. Sci. Lett. 223: 443-459.

Communicating editor: M. A. Beaumont 


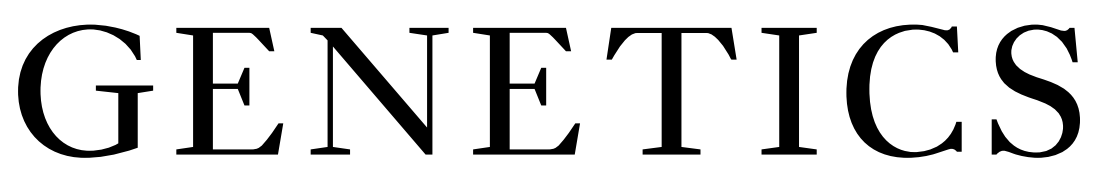

Supporting Information http://www.genetics.org/lookup/suppl/doi:10.1534/genetics.115.174425/-/DC1

\section{Examining Phylogenetic Relationships Among Gibbon Genera Using Whole Genome Sequence Data Using an Approximate Bayesian Computation Approach}

Krishna R. Veeramah, August E. Woerner, Laurel Johnstone, Ivo Gut, Marta Gut, Tomas Marques-Bonet, Lucia Carbone, Jeff D. Wall, and Michael F. Hammer 
File S1

\section{Supplementary Text}

\section{$2^{\text {nd }}$ Generation Sequencing}

Blood and tissues were obtained in agreement with protocols reviewed and approved by the Gibbon Conservation Center. DNA was extracted from blood or cell lines, and paired-end libraries were prepared with the Illumina TruSeq chemistry. Libraries were sequenced on the HiSeq 2000 platform, generating $2 \times 100$ bp reads. Four different sequencing centers contributed sequence data (Table S8). Multiple runs were performed to generate a minimum of 10X mean coverage on each sample after all processing. Mean coverage ranged from 11.5X to 19.5X (Table S9). Exome capture using the TruSeq Exome Enrichment Kit (Illumina) was performed on one NLE sample (Vok, 116x coverage) and one SSY sample (Monty, 64x coverage).

\section{Read Mapping and Variant Calling}

Sequences in FASTQ format were trimmed with cutadapt (MARTIN 2011) to remove Illumina TruSeq adapter sequences. Reads with less than 25 nucleotides left after trimming were dropped, along with their mates. The remaining reads were aligned to nomLeu1 with Stampy (v. 1.0.17) (LUNTER and GOodson 2011). For the two N. leucogenys (NLE) samples, Stampy was used in its "hybrid mode" where alignment with BWA (v. 0.5.9) (LI and DURBIN 2009) is attempted first. A substitution rate of 0.001 was specified, along with BWA minimum seed length of 2, fraction of missing alignments 0.0001 , and quality threshold 10 . For the non-NLE samples, stampy was used with a substitution rate of 0.015 (KIM et al. 2011). Local realignment at indel sites was performed with the Genome Analysis Toolkit (GATK, v. 1.4-37) (MCKENNA et al. 2010; DePRISTo et al. 2011). PCR duplicates were then removed with samtools. Picard (v. 1.70) (http://sourceforge.net/projects/picard/) CleanSam was run on the output. The two samples from each genus were then merged using Picard MergeSamFiles, and the resulting files were split using samtools (Ll et al. 2009) into 100 files containing 180 contigs each to facilitate further parallel processing. The GATK UnifiedGenotyper was run and Single Nucleotide Variants (SNVs) and indels with a quality score of at least 50 were retained to create a mask of variant sites to be excluded from base quality score recalibration (BQSR). The BQSR steps were run with the standard set of covariates, and the resulting files were merged across all samples. The GATK indel realignment tools were then run again to standardize alignment of indels across the samples. Default settings were used except that "BadCigar" reads were excluded and BAQ calculation was added. The UnifiedGenotyper from GATK version 2.1-11 was then used to call SNVs and indels in each genomic part using the "EMIT_ALL_SITES" mode (with the BAQ calculation included) to produce VCF files with data for all genomic positions. (Version 2.1 was used for this step to allow multiallelic calling). VCFs for all genomic parts were then merged 
using a custom perl script. Annotations were added to specify the consensus quality score of the nomLeu1 reference sequence at each position.

Exome sequencing data was processed separately from the shotgun data but using the same bioinformatic pipeline. The exome targeted regions were lifted over to the nomLeu1 genome using the UCSC liftOver utility with the default parameters, and the emit-all VCF of the exome capture data were restricted to these target loci. Sites within these loci with less than $30 x$ coverage or over $200 x$ coverage for any sample were also removed, while corresponding sites in the whole genome data with a variant quality less than 20 were called as homozygous reference in all samples.

\section{Finding Accurately Called Segregating Sites}

Machine learning classification techniques, such as variant quality score recalibration, have been successfully used to find a subset of sites that are predicted to be truly segregating in a sample. However, the authors know of no technique that has been used to predict whether or not individual genotypes have been correctly called, and as such downstream methods that presume that the genotypes are correct when they are in fact incorrect may suffer accordingly. To this end we developed a ML classification protocol to find a set of segregating sites where every genotype within is predicted to be correct for use in our Principal Components Analysis. Broadly, this protocol uses the comparison of the whole genome sequencing (WGS) and whole exome sequencing (WES) truth set to train several largely disparate classifiers. The classifiers are then used to predict the accuracy of individual genotypes across the genome. We note that this protocol may introduce some level of bias with respect to the agglomerative properties of sites (owing to the increased difficulty in calling heterozygous vs. homozygous genotypes) as opposed to individual genotypes, and as such this approach would be undesirable for evaluating, say, the site frequency spectrum.

More specifically, the machine learning (ML) suite Weka version 3.6.8 (HALL et al. 2009) was used to classify the whole genome genotype data at all called segregating sites, with the aim of finding a subset of very high quality sites. Using the definition of "correct" from our profiling of errors, we collected the set of all genotypes that were incorrectly called in the genome, and a random and equally sized sampling of genotypes that were called correctly for both our NLE and our non-NLE (SSY) sample. The following features were used in the machine learning analysis: approximate read depth, the next-best genotype likelihood, the haplotype score, the read-position bias score, the base quality rank score, the total mapping-quality 0 reads, the root-mean square mapping quality, the fraction of reads spanning deletions, the probability of strand bias, mapping quality rank sum test, quality by depth, the maximum likelihood expectation of the allele counts and allele frequency, the quality of the reference base, whether the call is from the NLE or the non-NLE sample, and the combined p-value of the 
distribution of read depths observed at the site. All but the last three features were taken directly from the GATK output. For the last feature, 2-tailed p-values of the read depth observed at a site for an individual were taken, based on that individual's empirical distribution of read depths, and these $p$-values were combined across individuals using the Method of Fisher to give a single description of read-depth for the site.

Using the features described above we generated a training set and evaluated the performance of a variety of classifiers using 10 -fold cross validation. Four techniques - multilayer perceptron, ridor, rotation forest and classification by regression-showed reasonable performance (75\%-85\% accuracy). However, as our goal is to find genotypes that have been correctly called, we used cost-sensitive classification to minimize our false discovery rate. Using a simple grid search, we found a cost-matrix that maximized each classifier's positive-predictive value by down-weighting the relative cost of false negatives versus false positives. 10 -fold cross validation gave the estimates of accuracy and positive predictive value shown in Table S10.

To reduce overfitting, each iteration from the 10 -fold cross validation for each learner type (e.g. rotation forest) was kept, with each of the folds being a function of the least-significant digit in the SNP position (e.g., Rotation Forest ${ }_{2}$ would be trained on all SNP positions where 2 is not its least-significant digit, and tested on all SNP positions where 2 is its leastsignificant digit). These $4 \times 10$ learners were then used in the assessment of genotype accuracy in our exome data. We then classified a genotype call as correct if all four classifiers predicted that the genotype was correct, and we classified a site as correct if all genotypes at a site were classified as correct. To increase our sample size of genotypes, the ML included sites that would have been masked out in the CNV calls (which represent less of a problem given that our measure of correctness is really a metric of consistency). Our final assessment of accuracy, however, included the CNV masks. Over a total of 54,528 sites that are segregating in the WES (after applying our filters) and marked as being genotyped correctly according to the 4 learners above, there was a total of 1 genotyping error and this error occurred in our non-NLE sample.

\section{Approximate Bayesian Computation analysis}

Sequence divergence essentially reflects an upper bound for when populations split and can give a false signal of the phylogeny if the time of coalescence for sequences can fall within the ancestral population of the extant populations of interest (DEGNAN and ROSENBERG 2006). Therefore in order to investigate the gibbon phylogeny at the population divergence level we applied a

Bayesian coalescent-based method that explicitly take into account sequence and population divergence simultaneously. Most methods that currently perform this task such as BEAST (DRUMMOND and RAMBAUT 2007) are not suited to large datasets that result from 2nd generation sequencing. Therefore we have developed an Approximate Bayesian Computation (ABC) (BEAUMONT et al. 2002) method that can cope with large amounts of sequence data, is not dependent on haplotype phase and can 
incorporate information derived from our modeling of errors from comparing WGS with high coverage WES data. We aimed to use the $A B C$ framework to a) identify the most likely species topology for the four gibbon genera that underwent WGS and b) estimate key parameters of the gibbon speciation process (specifically effective population sizes and divergence times).

\section{Methods}

Data: $A B C$ analysis was performed on two data sets. For the first, to approximate independence among regions we identified loci consisting of $1 \mathrm{~kb}$ of total callable sequence separated by at least $50 \mathrm{~kb}$. In addition to the masks and coverage filters described in the main manuscript we also masked $\mathrm{CpG}$ consistent sites as well as conserved phastCons elements inferred from primate genomes with a further $100 \mathrm{bp}$ padding either side of the element. Loci were then identified that were $50 \mathrm{~kb}$ from the nearest exon and where the 1,000 callable bases fall within a maximum of $3 \mathrm{~kb}$ of contiguous nomLeu1 reference sequence (i.e. callable bases are not necessarily contiguous) (see Fig S1 for a cartoon of the distribution of these loci). This resulted in 12,413 $1 \mathrm{~kb}$ loci (total of $\sim 12 \mathrm{Mb}$ ). Because these loci are relatively distant from each other ( $>50 \mathrm{~kb}$ apart) inter-locus linkage can be ignored and as they are relatively short $(\max 3 \mathrm{~kb})$ intra-locus recombination should be negligible. Therefore we do not incorporate recombination parameters into our simulations, only mutation plus the demographic parameters of interest. However because of the large number of loci analyzed, our data will approach the analytical expectations of the coalescent and thus should allow accurate and precise estimates of the correct model and associated parameters.

In addition we generated a set of $11,323200 \mathrm{bp}$ loci under the same criteria except the loci were orientated to lie on a known exon (i.e. genic versus non-genic loci) with the allowance of a maximum of $100 \mathrm{bp}$ either side of the known exon boundary, spanning a total of $4 \mathrm{~kb}$ with a minimum of $1 \mathrm{~kb}$ separating any two loci. This relatively small latter distance will likely violate the assumption of independent genealogies between loci somewhat but increasing this distance in to $5 \mathrm{~kb}$ severely reduced the number of loci, which will decrease accuracy and precision more readily. The choice of 200bp per loci for genic regions was motivated by the average length of exons in the gibbon genome of $213 \mathrm{bp}$. Variant sites were polarized against the aligned human reference genome, hg19, using the multiz 11-way alignments from UCSC.

Phylogeny Models and Parameter Priors: We treat all possible phylogenetic relationships amongst the four gibbon genera as distinct models (we also treat the two species within Hylobates as one population to reduce the model space). Therefore we need to consider a total of 15 models describing the population divergence relationship among the 4 genera, 12 asymmetric (Fig S15) and 3 symmetric (Fig S16). We also considered an instantaneous 4-way hard polytomy in a second ABC model testing analysis. As is standard for coalescent-based phylogenetic approaches the models are described by two classes of parameters, 
mean nucleotide diversity, $\theta$, and branch lengths in units of expected number of substitutions, $\tau$. Given an estimate of the mean mutation rate, $\mu$, the former can be transformed into an estimate of $\mathrm{N}_{\mathrm{e}}$ using $\theta=4 \mathrm{~N}_{\mathrm{e}} \mu$ and the latter can be transformed into a divergence time in generations, $t$, using $\tau=t \mu$. Priors ranged between $0.0001-0.03$ for all $\theta$ and $\tau$ parameters. Unless otherwise stated all prior distributions for all demographic parameters are all uniformly distributed on a $\log _{10}(\mathrm{x})$ scale. The justification for this prior range is that, assuming a mutation rate of $1 \times 10^{-8}$ per site per year and a 10 year generation time for gibbons, our individual priors are equivalent to a time of divergence of 100kya-30mya and an $N_{e}$ of 2,500-750,000. These ranges take into account the uncertainty we have with regard ape speciation times and ancestral diversity. Thirty million years sits at the upper end of the range when apes are thought to have diverged from other old world monkeys (ZALMOUT et al. 2010). The earliest known "sub-species" split times observed in great apes is 80kya (western and cross river gorilla), while the earliest known "species" split time (which is what our gibbon data essentially is) is 175kya (western and eastern gorillas), with most being much older (on the order of millions of years) (PRADO-MARTINEZ et al. 2013). Similarly, other estimates of great ape heterozygosity range from $\sim 0.0005-0.0025$, with ancestral $\theta$ estimates based on pairwise sequentially markovian coalescent (PSMC) analysis not exceeding 0.005, while we observed in a PSMC analysis of the same Gibbon samples used here (CARBONE et al. 2014) that the ancestral $N_{e}$ is unlikely to have risen to values greater than 50,000 .

When estimating parameters from the best model we included separate HPI and HMO populations with their own $\theta$ values and a new $\tau$ parameter for their divergence time. In addition we included a version of this model where the four genera split simultaneously, and thus only incorporate two ancestral $\theta$ parameters (one for the HPI and HMO ancestral population and one for the ancestral population of all four genera) and two $\tau$ parameters. Finally the analysis with this latter model was repeated using true uniform priors (rather than $\log _{10}$ transformed priors) for the two $\tau$ parameters (see Results in main manuscript for more details on this analysis)

Simulations: Coalescent simulations of the 8 individuals (16 chromosomes) were performed using a version of ms (Hudson) modified for Python that allowed fast parallel processing. In total we performed $10^{6}$ random draws of the parameter space and simulated a $\theta$-scaled genealogy for each locus. In order to account for mutation rate heterogeneity across loci we estimated relative sequence divergence for all loci, taking the average sequence divergence for each of the eight gibbon individuals from hg19. These individual locus estimates were then normalized around a mean of 1, allowing us to follow the approach of Rannala and Yang (RANNALA and YANG 2003) and scale $\theta$ for each individual locus in our demographic simulations. 
Stochastic Error Modeling: We used the error profiles for the singleton and non-singleton categories described above in Vok and Monty to construct an error model $E=\langle S, M\rangle$ for a particular sample that could transform perfectly correct data generated by coalescent simulations into data reflective of the error processes that are likely to have occurred during whole genome sequencing and post processing. We found that with our bioinformatic pipeline the total number of observed singletons was always less than or equal to the true number. Therefore $S$ was calculated as the proportion of missing singletons, or the probability of not calling a true singleton in the WGS data. During a coalescent simulation of genetic data $S$ reflects the rate at which true singletons will be hidden or dropped and the genotype called as homozygous reference. To construct $M$ we took the $3 \times 3$ confusion matrix generated for non-singletons and divided the number in each element of the matrix by the sum of all elements within their respective columns. During a simulation of genetic data, for any site not classed as a true singleton but still segregating, the values within a particular column of $M$ reflect the probabilities of a multinomial distribution that determines the rate that a true genotype of a particular type will be transformed to one of the two other genotypes or stay the same.

To apply our error correction to a) non-exome regions in the two target samples and b) non-exome regions in the other six samples for which there was no WES we constructed separate $E$ models for each read depth $\geq 7 \mathrm{X}$ (i.e, we constructed $E_{i}=\left\langle S_{i}, M_{i}\right\rangle$, where $E_{i}$ is the estimated error at read-depth i). Singleton calling was markedly better in the reference taxon (S 99\%), then in the non-reference taxon (S 96\%). For $S_{i}$, error rates initially decreased up to $\sim 20 x$ but past 30 showed substantial increases in errors, presumably from uncalled CNVs (Fig S17). Similar to our findings with singletons, WGS/WES discordance rates initially decreased with increasing read depth, but from read depths of $\sim 20 x$ onwards discordance rates again began to increase (Fig S18). Given the error profiles observed with respect to coverage, we chose to break our error rate estimations into three read-depth classes; 7-20x, 21-29x and $\geq 30 x$. For the first class, we assumed that our per-read-depth estimates were correct, and for the last class, consistent with our assumption from the WES data, that the WGS calling was perfect. For the middle class, however, we conservatively assumed a constant error rate taken from the average error rate from read-depths $18-20$.

This information allowed us to construct an overall $E$ model for a particular sample, regardless of whether it was one of the two target samples or not, by taking a weighted average of $E_{i}$, with weights determined by the empirical distribution of read depths at the specific regions of interest for sites between $7 x$ and that individual's $95^{\text {th }}$ percentile of read depth. The $E_{i}$ models for Vok and Monty were used for NLE and non-NLE samples respectively to take into account any mapping biases. We assumed errors were uncorrelated between individuals. As the error models were generated with respect to the nomLeu1 reference (rather than some ancestral reference) we simulated an additional haploid NLE sample to orient the error correction 
appropriately. Summary statistics were generated from the simulations for both with and without stochastically modeling error processes in order to examine the affect of the former.

Ancestral state misidentification adjustment: $2 \%$ ancestral state misidentification was incorporated into simulations by calculating the expected number of sites to experience a mutation along the hg19 lineage for each locus $(1000 \mathrm{bp} \times 2 \%=20$ sites). The number of sites to actually "flip" (i.e. assign the wrong ancestral state) for each locus during a simulation is drawn from a Poisson distribution with this mean. These sites are then randomly assigned to positions along the locus, though only positions that are found to segregate amongst the gibbon chromosomes need to be flipped.

Summary Statistics: We computed the following summary statistics to describe the data for every pair of populations: number of shared derived polymorphisms across loci, number of private derived polymorphisms in each population and the number of private fixed sites in each population (Table S11). These statistics are known to contain substantial information about population demography (WAKELEY 1996) and are utilized in the program MIMAR (BECQUET and PRZEWORSKI 2007). These statistics are particularly useful in the case of short read sequence data as they do not require haplotype inference. We use the mean of these summary statistics across all loci to describe the data (unlike MIMAR where these summaries are used to calculate a likelihood of the data for each locus individually, which is computationally intensive for the amount of data considered here). We also explored the use of the variance of these same summary statistics across loci but found they added little to our ability to infer parameters in the model while contributing more noise to the partial least squares (PLS) transformation and reducing the proportion of correctly inferred simulated topologies using simulated data (see below). Other summary statistics that might traditionally be considered useful for demographic inference such as Tajima's D were not utilized due to the small sample size for each species. Therefore our method is unable to infer parameters such as population growth rates.

Inference: $\mathrm{ABC}$ analysis was performed using two different regression adjustments depending on their application. When estimating model parameters we utilized ABCtoolbox (WEGMANN et al. 2010), which implements a general linear model (GLM) adjustment (LeUENBERGER and WEGMANN 2010) on retained simulations. The GLM adjustment, by modeling the parameters as the predictor rather than the response variable, avoids one particular limitation of the standard linear regression adjustment of Beaumont et al. (BEAUMONT et al. 2002) where the posterior distribution can end up being non-zero in parameter space that actually lies outside the prior bounds. To maximize sufficiency but limit dimensionality, the full set of summary statistics was transformed into PLS components (WEGMANN et al. 2009) and we used the change in Root Mean Square Error (RMSE) to guide 
the choice of number of components. These PLS components were then used to estimate parameters. In our analysis to assess the ability of our $A B C$ framework to determine the correct species topology/model (see below) we compared the marginal distributions across models using both the GLM adjustment above and the multinomial logistic regression (LR) method previously described by Fagundes et al. (FAGUNDES et al. 2007). The performance of the two methods was generally very similar though the LR method demonstrated a slight increase ( $3 \%)$ in the proportion of correctly recovered models. Because of this slightly better performance, added to the fact the LR method is by far the most popular regression adjustment method used for ABC model choice (CSILLÉRY et al. 2010) and we are less concerned in this case with extrapolating the posterior distributions outside of the prior range (as we are using categorical classes and all classes have equal prior probability), we chose to use this method for all subsequent analysis using an adapted version of the R function calmod.r as. However the use of either method is likely to give very similar results in our particular framework. $1 \%$ of simulations were retained for the GLM (parameter estimation) and LR (model choice) adjustments. PCA was used for comparing the multidimensional distribution of summary statistics using the "prcomp" function in R.

Using simulated data to assess the ability to determine the correct species topology

In order to assess the reliability of our method to infer the correct species tree from a set of alternatives we simulated 10,000 random pseudo-observed datasets from our model and demographic parameter priors and attempted to recover the true topology using the $A B C$ machinery. We explored which combination of summary statistics most often inferred the correct topology and found that the six summary statistics describing the mean number of shared sites for a pair of populations was most effective. Adding more summary statistics (such as mean pairwise fixed or private differences or the variance of the number of shared sites across loci) reduced the proportion of correctly inferred simulated topologies and thus were discarded for this analysis.

Using the LR method for the error-corrected non-genic data we recovered the correct model $88.4 \%(7,989 / 9,042)$ of the time (for 958 topologies the LR method failed to converge), the correct model was one of the top 3 models $99.1 \%$ $(8,959 / 9,042)$ of the time and had a posterior probability greater than $5 \% 98.3 \%(8,894 / 9,042)$ of the time. Using a more naïve method (the direct method, DR) of the proportion of retained simulations from each model (PRITCHARD et al. 1999) we recovered the correct model $77.6 \%(7,757 / 1,000)$ of the time, the correct model was one of the top 3 models $96.7 \%$ $(9,673 / 10,000)$ of the time and had a posterior probability greater than $5 \% 99.5 \%(9,950 / 10,000)$ of the time. For the 958 occasions when the LR method failed, the DR method inferred the correct model 792 (83.0\%) times and was within the top 3 models on every occasion bar one, with a minimum posterior probability of 0.07 . This suggests the failure of the LR method to 
converge results from either complete separation or because all the retained simulations are only from one model, rather than an inability to detect the correct model. The posterior probabilities using both the LR and DR methods were highly informative with regard to the correct model. However the LR method generally demonstrated a better level of discrimination between the true model and all other models (Fig S4). Therefore we decided to use this method for all subsequent analysis. For the uncorrected data, there was a slight increase in the ability to infer the correct topology (which is unsurprising given the error model essentially adds noise) where, for example, using the LR method we recovered the correct model 8,298/9,048 (91.7\%) of the time (the 952 topologies for the LR method failed). The proportion of occasions where we inferred the correct topology for corrected genic data was similar (86.9\%).

In order to obtain a better idea of where our method failed (and where it performed well) we performed a more targeted set of simulations and again attempted to infer the correct model using our ABC framework. We first chose the total height of the four taxa species tree (Tanc) to be one of three values (in units of mutations per site): $0.01,0.005$ and 0.001 . Assuming a mutation rate of $1 \times 10^{-9}$ per site per year this is equivalent to 10,5 and 1 million years. We then chose the $\vartheta$ values across the tree to be either fixed at 0.001 in all present and past populations, or for the present values to be $0.0012,0.0004,0.002,0.0008$ (thus roughly reflecting present day estimated $\vartheta$ for gibbons in this study) and for the ancestral populations to reflect the combinations of these $\vartheta$ values (i.e. $0.0012+0.0004=0.0016,0.0016+0.002=0.0036,0.0036+0.0008=0.0044$, thus the $\mathrm{N}_{e}$ gets increasingly bigger going back in time to increase the probability of incomplete lineage sorting in the ancestral populations, i.e. we make the problem "harder"). The purpose of the latter set of $\vartheta$ values is not to choose values that necessarily reflect reality (though we attempt to pick sensible choices that will prove intuitively useful), but to examine how the method tolerates changes in $\theta$ compared to utilizing fixed values (which should be an "easier" problem). Finally we simulated either an asymmetrical tree or symmetrical tree. Thus there are 12 parameter combinations representing 12 scenarios that define our simulations. For each of these 12 scenarios we choose the two most recent divergence times (Tanc is the third and last event and is already set) over a range as follows.

1) The most recent divergence event is chosen to be equal to Tanc/ $\alpha$, where $\alpha$ varies from $1.1-5$, with steps of 0.1 . The smaller $\alpha$, the closer the most recent divergence event will be to the final divergence event. For example for $\alpha$ of 1.1 , when Tanc $=0.001$ this means that the most recent divergent event occurs at 0.0009, which results in a separation time of only $\sim 100,000$ in years.

2) The second divergence event is chosen based on 6 , which ranges between 0.01-0.99 in steps of 0.01, with the value of $B$ reflecting the distance from the final divergence event as a percentage of the time between the first and last 
divergence event. Again, a smaller $B$ reflects a second divergence event that is very close to the last divergence event (conversely a high $B$ reflects a divergence event that is very close to the first divergence event).

We used our $A B C$ machinery to determine the posterior probabilities for the true model under these 12 scenarios while varying $\alpha$ and $B$ along a two dimensional grid. Posterior probabilities were determined using either the DR or LR method. Fig S5 shows these results with the grid of $\alpha$ and $B$ being the $X$ and $Y$ axis and the surface of the posterior probabilities across this grid shown in the Z-axis. It is immediately apparent that the LR produces much higher posterior probabilities compared to the DR method, with much of the surface of the former being at or close to 1.0. In both cases reducing Tanc to 0.001 reduces the posterior probability, but the affect is markedly worse for the DR method. Unsurprisingly, for the asymmetric model our ability to infer the true model is best when $\alpha$ is highest and $B$ is 0.5 and for the symmetric model when $\alpha$ is highest and 6 is 1 . This essentially reflects situations where the divergence events are maximally separated in terms of branch lengths. The LR method performs particularly well in most cases, with the posterior probability only decreasing markedly at the edges of the grid (and for Tanc 0.001 and 0.005 the value of $\alpha$ has almost no effect) suggesting that for a Tanc realistic for gibbons (>4Mya), the method will only perform sub optimally if the second divergence event is very close to the first or last divergence event, and even then the posterior probability will still likely be one of the higher values across all 15 possible topologies. Varying $\theta$ across populations (annotated as fixed in Fig S5) does not appear to have a large effect for the DR or LR methods but does appear to further exacerbate the poorer performance of the method when Tanc $=0.001$, where we presume incomplete lineage sorting becomes particularly prevalent such that it obscures true tree topology.

\section{Using simulated data to assess the effect of stochastically modeling error processes on parameter inference}

To assess how stochastically modeling errors in our simulations for $A B C$ analysis are likely to affect inference of parameters, we applied our entire analysis framework to simple demographic scenarios where the data was simulated to mimic errors in next generation sequencing that occur as a result of variable per site coverage and read-specific base miscalling. Specifically we assessed the affect of our analysis strategy for estimating parameters under two demographic scenarios (see Fig S19):

Scenario A. We estimate $\theta$ in a one-population of constant size model. We sample two individuals (four chromosomes) from the population, one of which is used as a target sample for which we know the true genotypes to generate an $E$ model, as well as a reference chromosome.

Scenario B. We estimate $\tau$ in a two-populations of constant size with divergence model, where the $\theta$ values in the two present day populations and the ancestral population are fixed at $0.001,0.002$ and 0.001 respectively. We sample two individuals from 
each population (eight chromosomes), with one from each used as a target sample for which we know the true genotypes to generate an $E$ model. The reference genome is drawn from the first population.

Our framework for these analyses for a particular demographic model (with a specifc parameter value of interest) involves four primary stages (further details are given below):

1. Simulating medium coverage next generation sequence data in "exome regions" under the demographic model of interest and calling genotypes from this data, which along with the known true genotypes allows the construction of $E_{i}$ models for target samples at individual read depths $\geq 7 \mathrm{X}$.

2. Simulating medium coverage next generation sequence data in "neutral regions" under the demographic model of interest and calling genotypes from this data. This is essentially our observed data for the ABC analysis.

3. Constructing overall $E$ models for all individuals based on their simulated empirical distribution of read depths at the neutral regions and the $E_{i}$ from the target samples in stage 1.

4. Performing an $A B C$ analysis to infer parameters where we stochastically introduce errors into the $A B C$ Monte Carlo simulations based on the $E$ model constructed for each sample.

In theory we should apply all steps for every parameter value we would like to explore under a given demographic scenario. We aimed to examine scenarios $A$ and $B$ for $\theta$ and $\tau$ values that ranged from 0.0001 to 0.01 in steps of $0.1 \log _{10}$ units. This would involve applying our framework 21 times for each scenario. Even for this relatively modest exploration of the parameter space, this would be particularly time consuming for stage 4 where we must generate hundreds of thousands of Monte Carlo simulated datasets each time. However we have found that error profiles to generate $E$ models are only minimally affected by the specific $\theta$ or $\tau$ used in our two particular demographic scenarios (they become much more variable in more complex demographic scenarios, data not shown) (Fig S20). Therefore, in practice we construct our read depth specific $E_{i}$ and overall $E$ models (steps 1 and 3) and generate Monte Carlo simulations (step 4) only for $\theta$ or $\tau=0.001$ (i.e. we utilize the midpoint parameter value) and always apply the same empirical distribution of read depths for each sample for neutral regions regardless of the value of $\theta$ or $\tau$ (step 2). Therefore, we only need to perform steps 1,3 and 4 once across all $\theta$ or $\tau$ values for a particular demographic scenario, though it is still necessary to perform step 2 to generate the observed data for each of the individual 21 parameter values. This will mean our $A B C$ analysis is only optimally applied to the parameter value of 0.001 and all other inference will be slightly sub-optimal compared to how the method could be applied in practice. 
Simulating the truth set: For a given demographic model and parameter value of interest we simulated $20,000 \times 8$ exons each of length $150 \mathrm{bp}$ for the appropriate number of chromosomes given the model (including a reference chromosome) using ms. This gives a total diploid sequence length of $24 \mathrm{MB}$ per individual, approximating the amount of data generated by most WES capture kits. This simulated sequence data reflects the true genotypes and essentially represent the high coverage WES data from Vok and Monty in our analysis of real data.

Simulating next generation sequence data in exomic regions: To simulate medium coverage next generation sequencing data for these same true underlying genotypes, we assume each individual was sequenced to a mean coverage of $10 \mathrm{X}$ and each site for each individual is assigned some number of reads (i.e. coverage) by randomly drawing from a poisson distribution with $\lambda=10$. For each true heterozygous site the number of reads "sequenced" for each allele is drawn from a binomial distribution with $p=0.5$. Each read at a site is assigned a Phred-scaled quality score, $Q$, from a truncated poisson distribution with $\lambda=30$ such that $Q$ s are repeatedly drawn until a value $\leq 40$ is obtained (i.e. $Q$ is limited to 40 ) and an error is introduced at a rate proportional to this $Q$ value (for example for reads with $Q=30$ there is a 1 in 100 probability that it will be assigned the wrong base call). In keeping with our use of the infinite sites model in $m s$ and to simply our downstream analysis we limit bases to only two types, reference and non-reference, rather than he four bases A, C, T and G.

In reality there is considerably more complexity with regard to how coverage and base calling error is distributed across the genome than is considered in our framework. Coverage is necessarily correlated at sites because reads span at least $100 \mathrm{bp}$ of sequence and paired end reads are used in most circumstances, while sequencing errors tend to be more frequent at the end of reads. In addition $Q$ values assigned by Illumina sequencing software are frequently not truly representative of the true error rate and are base pair and context dependent. In addition there may also be differences in the proportion of reads that correctly map to the reference genome when utilizing a mixture of reference on non-reference species individuals. We also do not consider the effect of Indels or repeats and CNVs, which can introduce additional error from misalignment.

Calling genotypes from the simulated next generation sequence data: We recoded the maximum likelihood genotype and Bayesian variant calling algorithms described in Depristo et al.(DepRISTo et al. 2011) to only consider two alleles and called genotypes (assuming a heterozygosity parameter of 0.001) from our simulated next generation sequence data for any sites with coverage $\geq 7 x$. Sites with a variant quality value $<40$ were assigned as homozygous reference in all samples. These called genotypes essentially represent the medium coverage WGS data in our error modeling. 
Construction of $E_{i}$ for target samples: Given these simulated WGS and truth data sets we can construct $E_{i}$ for target samples as described for the real data. When examining error profiles for both scenarios we see a consistent pattern of a decreased proportion of missing singletons with increased coverage, with values of $\sim 15 \%$ missing singletons at $7 X$ but rising to no errors by 20X (Fig S20). This trend is largely in line with our real data (Fig S17), suggesting that our framework is capturing the most important error processes, though the real data is much noisier which is likely due to many of the additional factors described above.

Simulating next generation sequence data in neutral regions: To simulate "neutral regions" (the observed data) we use the same simulation framework described above to introduce errors and generate called genotypes for the exomic regions except that we simulate $12,0001 \mathrm{~kb}$ regions (to mimic our real data of $1 \mathrm{~kb}$ regions). $E$ models are then constructed for each sample given a simulated distribution of coverage in the neutral regions.

$A B C$ inference: We then perform two separate $A B C$ analyses, one with the introduction of stochastic errors via the $E$ model and one without. For Scenario A we use the mean number of segregating sites per locus as the summary statistic and for Scenario B we use the mean number shared, private and fixed sites per locus between the two populations.

Results: The framework described above (subjective to some simplifications to aid tractability) was applied for a range of $\theta$ and $\tau$ values under scenarios A and B respectively. We then compared our estimated parameter values (using the mode and median of the posterior distributions) to the true simulated value. The use of the $E$ model consistently improves the estimate of parameters, with the effect being particularly noticeable for larger value of $\tau$ (Fig S6). For example when the true $\tau$ is 0.010 , modeling errors results in almost no difference with the estimate $\tau(\tau=0.0098)$ compared to when errors are ignored $(\tau=$ 0.0079). In units of time in years this is a difference of $\sim 2 \mathrm{my}$. The RMSE for $\theta$ and $\tau$ when using the $E$ model is $36 \%$ and $8 \%$ of that respectively when not using the $E$ model. Thus our simulations suggest that stochastic modeling of error processes in $A B C$ simulations can improve the inference of parameters for $2^{\text {nd }}$ generation sequencing data.

\section{G-PhoCS analysis}

The Markov Chain Monte Carlo (MCMC) Bayesian coalescent-based method described by Gronau et al. (GronAU et al. 2011) was performed using the software G-PhoCS to estimate $\theta$ and $\tau$ values for a bifurcating tree (we ignored the effect of migration). On this occasion we included a human haploid sequence (hg19) as an outgroup for the overall gibbon phylogeny. The same 12,431 
$1 \mathrm{~kb}$ loci and assumed bifurcating tree from the $A B C$ analysis described above were utilized and the mutation rate was fixed individually for each locus as above using the normalized divergence values. The gamma prior for $\theta$ was set to be relatively broad and the same for all present and ancestral populations with shape, $\alpha,=2$ and rate, $\beta,=1,000$. Gamma priors for $\tau$ were also set to be relatively broad, with the $\alpha$ value always 2 . However, either $a$ ) $\beta$ was set as 200 for all $\tau$ values such that the mean $(\alpha / \beta)=0.01$, which, when assuming $\mu=1 \times 10-9 /$ site/year equates to $10 \mathrm{My}$ or $b$ ) individual $\beta$ values were set for each $\tau$ such that the mean value reflected rough estimates from the $A B C$ analysis or for the human/gibbon split time from Carbone et al. (CARBONE et al. 2014) (Table S1). Starting values for the MCMC chain for each parameter were chosen randomly from the interval of $0.8-1.2 *$ these mean values. Preliminary runs under $b$ ) were used to tune the MCMC mixing (as this is the multidimensional parameter space that is likely to be most important for estimating parameters in this case and mixing properties can change in different parts of the space), such that the rate of acceptance was between $20 \%-70 \%$ for all parameters of the model.

Once we obtained good mixing properties we ran three independent MCMC chains for both prior settings $a$ ) and $b$ ) for a total of six chains. We allowed 10,000 samples as burn-in followed by 100,000 samples for estimating parameters (this sample size should be large enough that we do not require independent samples to get unbiased estimates due to correlation among consecutive samples). The Markov chain converged to stationarity much quicker than the utilized burn-in period, and all six runs converged to the same tationary distribution, though prior setting a) required slightly more samples as the starting positions were further away from the converged $\tau$ values. Results were processed using the software Tracer (http://tree.bio.ed.ac.uk/software/tracer/).

\section{References}

BeAumont M. A., ZhANG W., BaLding D. J., 2002 Approximate Bayesian computation in population genetics. Genetics 162: 20252035.

BeCQuet C., PRZEWORSKI M., 2007 A new approach to estimate parameters of speciation models with application to apes. Genome Res 17: 1505-1519.

Carbone L., Harris R. A., Gnerre S., Veeramah K. R., 2014 Gibbon genome and the fast karyotype evolution of small apes. Nature.

CSILléry K., Blum M., GAGgIOTTI O. E., AL E., 2010 Approximate Bayesian computation (ABC) in practice. Trends in ecology \& .... 
Degnan J. H., Rosenberg N. A., 2006 Discordance of Species Trees with Their Most Likely Gene Trees. PLoS Genet 2: e68.

Depristo M. A., Banks E., Poplin R., Garimella K. V., Maguire J. R., Hartl C., Philippakis A. A., del Angel G., Rivas M. A., Hanna M., Mckenna A., Fennell T. J., Kernytsky A. M., Sivachenko A. Y., Cibulskis K., Gabriel S. B., Altshuler D., Daly M. J., 2011 A framework for variation discovery and genotyping using next-generation DNA sequencing data. Nat Genet 43: 491-498.

Drummond A. J., RAMBAUt A., 2007 BEAST: Bayesian evolutionary analysis by sampling trees. BMC Evol Biol 7: 214.

Fagundes N. J. R., Ray N., Beaumont M., Neuenschwander S., Salzano F. M., Bonatto S. L., Excoffier L., 2007 Statistical evaluation of alternative models of human evolution. Proceedings of the National Academy of Sciences 104: 17614-17619.

Gronau I., Hubisz M. J., Gulko B., Danko C. G., Siepel A., 2011 Bayesian inference of ancient human demography from individual genome sequences. Nat Genet 43: 1031-1034.

Hall M., Frank E., Holmes G., Pfahringer B., Reutemann P., Witten I. H., 2009 The WeKA data mining software. SigkDD Explor. Newsl. 11: 10.

Kim S. K., Carbone L., Becquet C., Mootnick A. R., Li D. J., de Jong P. J., Wall J. D., 2011 Patterns of Genetic Variation Within and Between Gibbon Species. Molecular Biology and Evolution 28: 2211-2218.

Leuenberger C., Wegmann D., 2010 Bayesian Computation and Model Selection Without Likelihoods. Genetics 184: $243-252$.

LI H., DURBIN R., 2009 Fast and accurate short read alignment with Burrows-Wheeler transform. Bioinformatics 25: 1754-1760.

li H., Handsaker B., Wysoker A., Fennell T., Ruan J., Homer N., Marth G., Abecasis G., Durbin R., 1000 genome Project Data Processing Subgroup, 2009 The Sequence Alignment/Map format and SAMtools. Bioinformatics 25: 2078-2079.

LUNTER G., GOODSON M., 2011 Stampy: A statistical algorithm for sensitive and fast mapping of Illumina sequence reads. Genome Res 21: 936-939.

MARTIN M., 2011 Cutadapt removes adapter sequences from high-throughput sequencing reads. EMBnet.journal 17: pp. 10-12.

Mckenna A., Hanna M., Banks E., Sivachenko A., Cibulskis K., Kernytsky A., Garimella K., Altshuler D., Gabriel S., Daly M., Depristo M. A., 2010 The Genome Analysis Toolkit: a MapReduce framework for analyzing next-generation DNA sequencing data. Genome Res 20: 1297-1303. 
Prado-Martinez J., Sudmant P. H., Kidd J. M., Li H., Kelley J. L., Lorente-Galdos B., Veeramah K. R., Woerner A. E., O'Connor T. D., Santpere G., Cagan A., Theunert C., Casals F., laayouni H., Munch K., Hobolth A., Halager A. E., Malig M., Hernandez-Rodriguez J., Hernando-Herraez I., Prüfer K., Pybus M., Johnstone L., Lachmann M., Alkan C., Twigg D., Petit N., Baker C., Hormozdiari F., Fernandez-Callejo M., Dabad M., Wilson M. L., Stevison L., Camprubí C., Carvalho T., Ruiz-Herrera A., Vives L., Melé M., Abello T., Kondova I., Bontrop R. E., Pusey A., Lankester F., Kiyang J. A., Bergl R. A., Lonsdorf E., Myers S., Ventura M., Gagneux P., Comas D., Siegismund H., Blanc J., Agueda-Calpena L., Gut M., Fulton L., Tishkoff S. A., Mullikin J. C., Wilson R. K., Gut I. G., Gonder M. K., Ryder O. A., Hahn B. H., Navarro A., Akey J. M., Bertranpetit J., Reich D., Mailund T., Schierup M. H., Hvilsom C., Andrés A. M., Wall J. D., Bustamante C. D., Hammer M. F., Eichler E. E., Marques-Bonet T., 2013 Great ape genetic diversity and population history. Nature 499: 471-475.

Pritchard J. K., Seielstad M. T., Perez-Lezaun A., Feldman M. W., 1999 Population growth of human Y chromosomes: a study of Y chromosome microsatellites. Molecular Biology and Evolution 16: 1791-1798.

RANNALA B., YANG Z., 2003 Bayes estimation of species divergence times and ancestral population sizes using DNA sequences from multiple loci. Genetics 164: 1645-1656.

WAKELEY J., 1996 Distinguishing Migration from Isolation Using the Variance of Pairwise Differences. Theoretical Population Biology 49: 369-386.

Wegmann D., Leuenberger C., Excoffier L., 2009 Efficient Approximate Bayesian Computation Coupled With Markov Chain Monte Carlo Without Likelihood. Genetics 182: 1207-1218.

Wegmann D., Leuenberger C., Neuenschwander S., Excoffier L., 2010 ABCtoolbox: a versatile toolkit for approximate Bayesian computations. BMC Bioinformatics 11: 116.

Zalmout I. S., Sanders W. J., Maclatchy L. M., Gunnell G. F., Al-Mufarreh Y. A., Ali M. A., Nasser A.-A. H., Al-Masari A. M., Al-Sobhi S. A., Nadhra A. O., Matari A. H., Wilson J. A., Gingerich P. D., 2010 New Oligocene primate from Saudi Arabia and the divergence of apes and Old World monkeys. Nature 466: 360-364. 
Exon

$\square$ Non-genic loci

Genic loci

Filtered or masked sequence

NLE ref

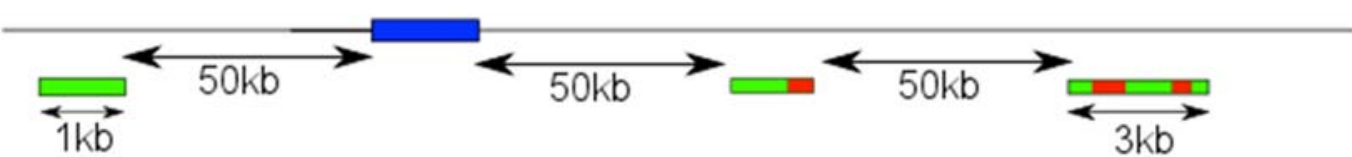
identification and distribution of non-genic loci

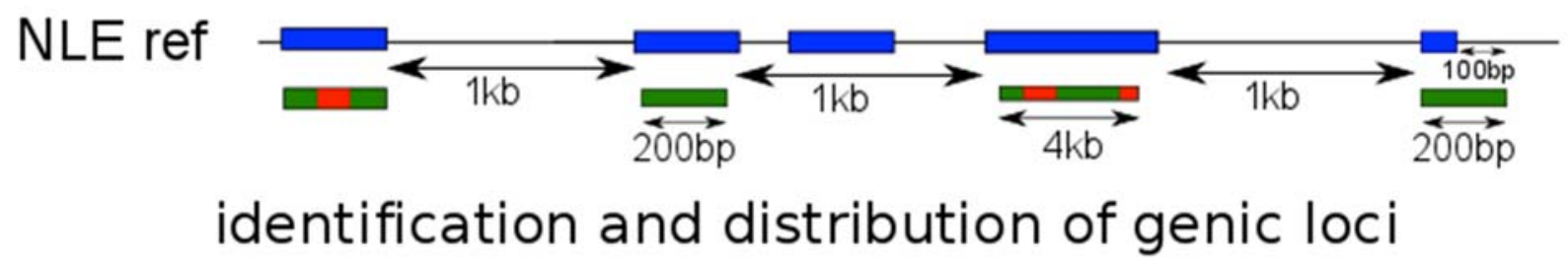

Figure S1 Cartoon showing the distribution of genic (200bp) and non-genic (1kb) loci identified for phylogentic analysis of gibbons. Not to scale. 


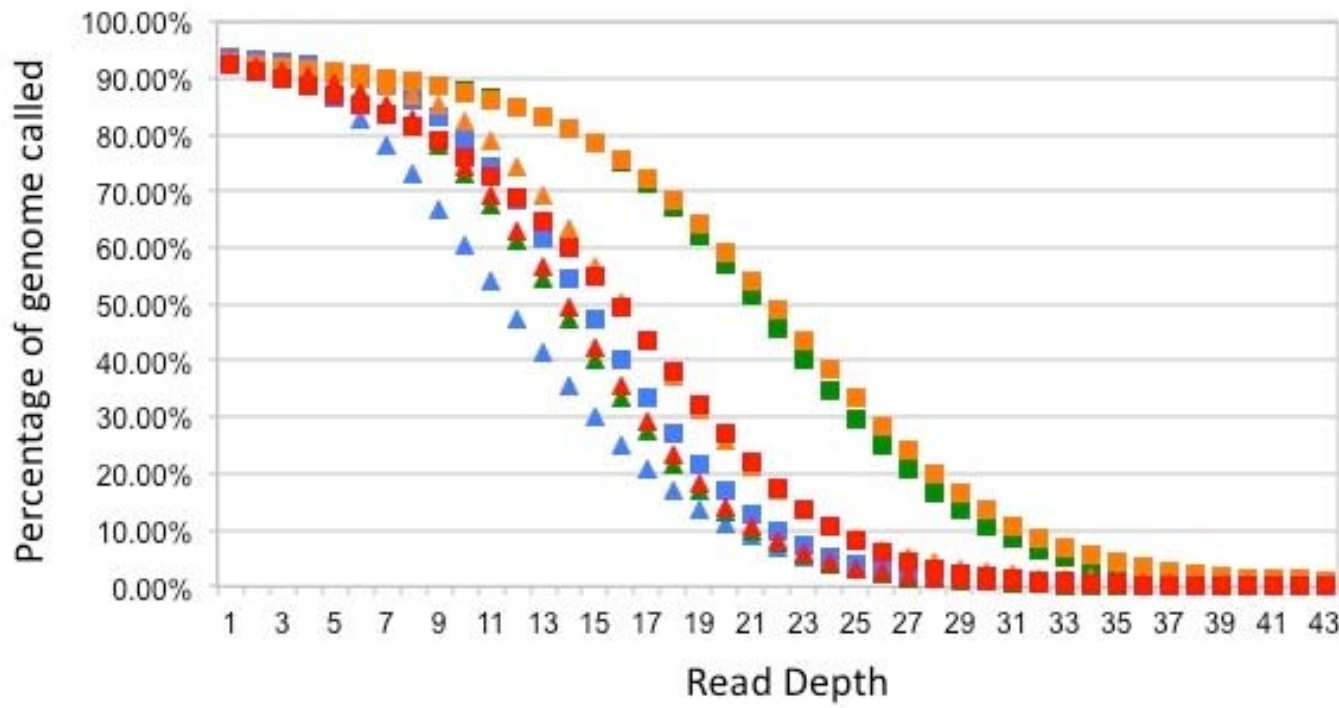

- NLE_Vok $\triangle$ NLE Asteriks ASSY Monty aSSY_Karenina $\triangle$ HLE Drew = - HPI Domino

AHMO_Madena

Figure S2 Distribution of coverage in the 8 gibbon samples 

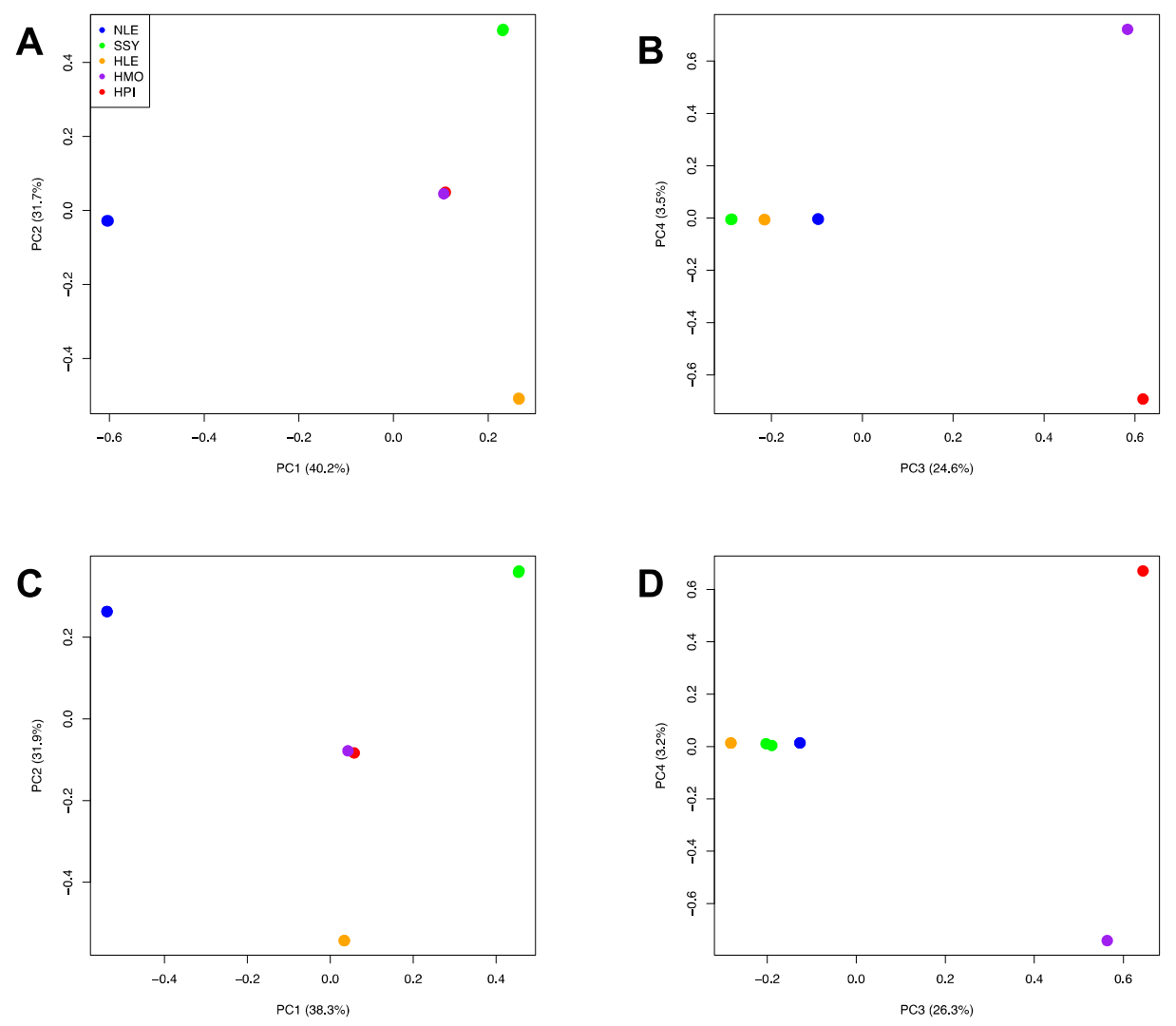

Figure S3 PCA of all 8 gibbon samples based on high quality genotypes. A) PCA 1v2 for all SNPs, B) PCA 3v4 for all SNPs, C) PCA 1v2 for random 1\% of SNPs, D) PCA 3v4 for random 1\% of SNPs. Note the two individuals from NLE, SSY and HLE appear as one point on the plot as their PC coordinates were highly similar relative to between species differences. 


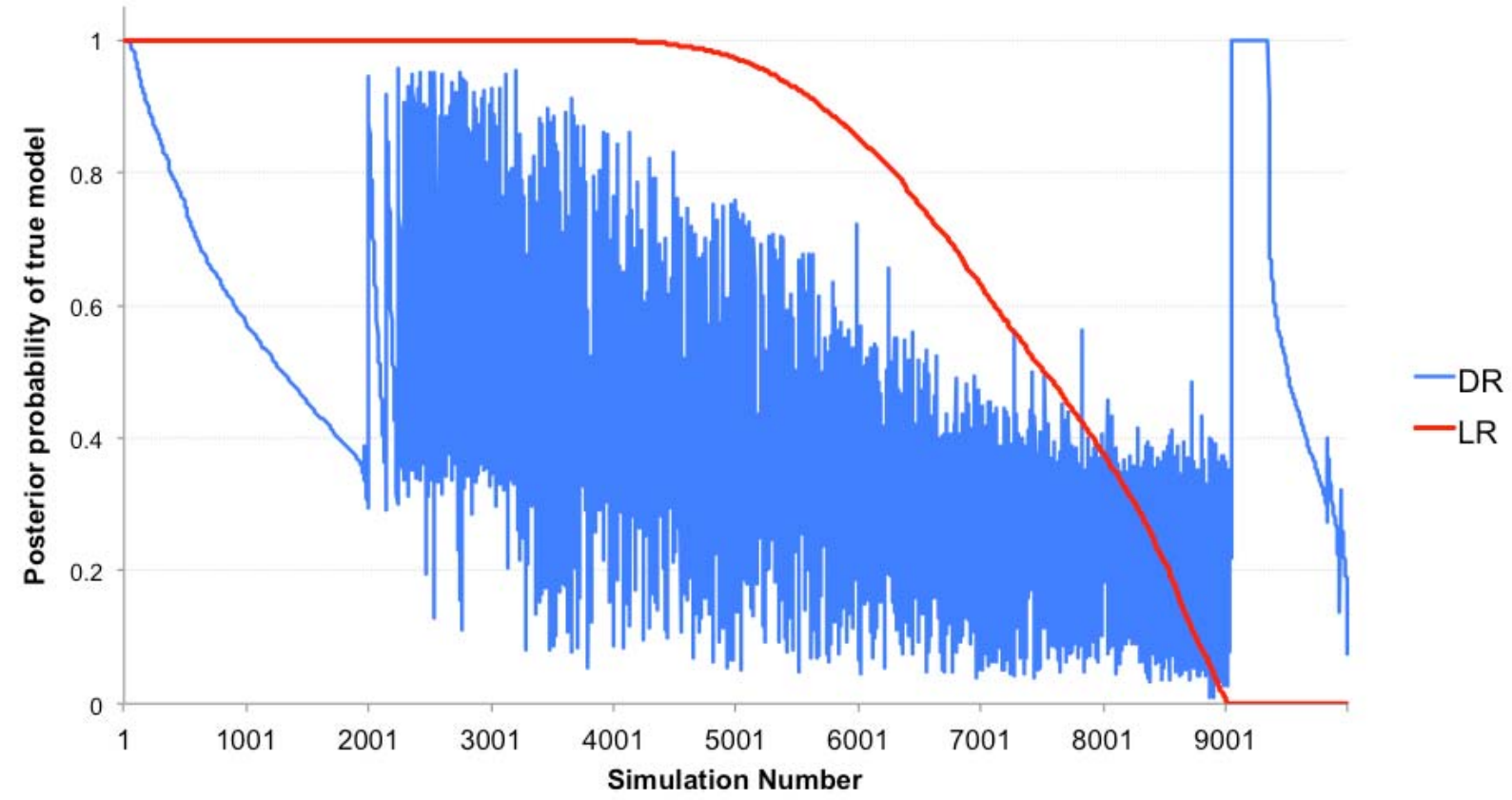

Figure S4 Relative posterior probabilities as assessed by our ABC framework for 10,000 random simulated topologies (from a total of 15 possible topologies for 4 genera) using the Logistic Regression (LR) and Direct (DR) methods. Simulations are ordered from highest to lowest LR posterior probabilities. 
Asymmetric tree, $\theta=0.001 \mathrm{DM}$
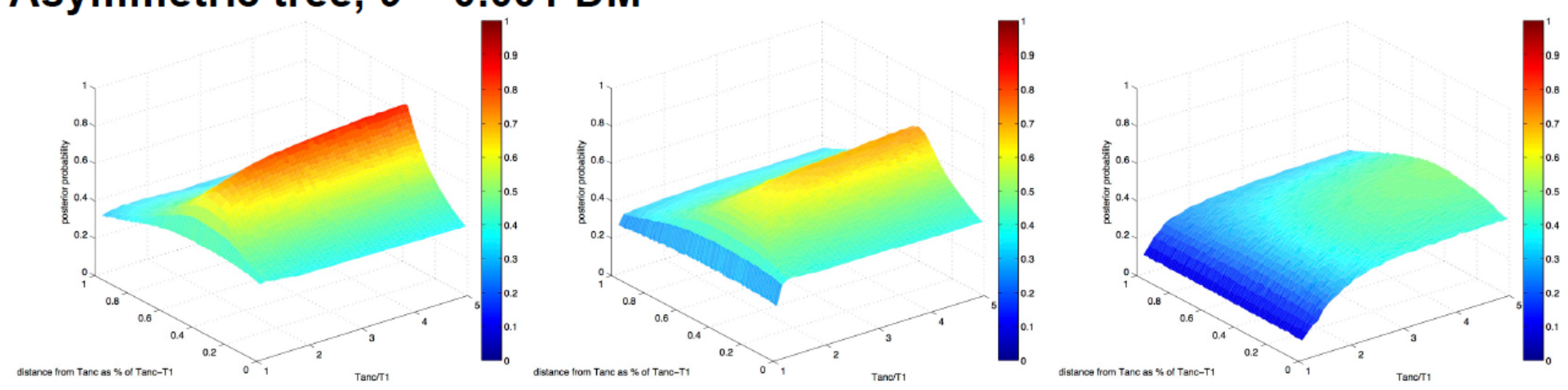

Symmetric tree, $\theta=0.001 \mathrm{DM}$

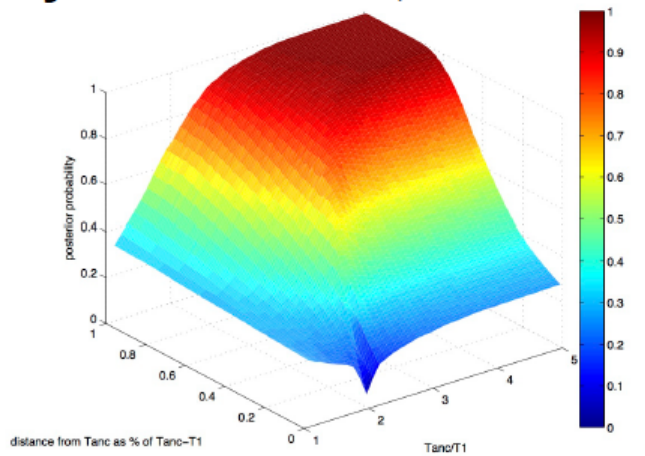

Tanc $=0.01$

10 Mya

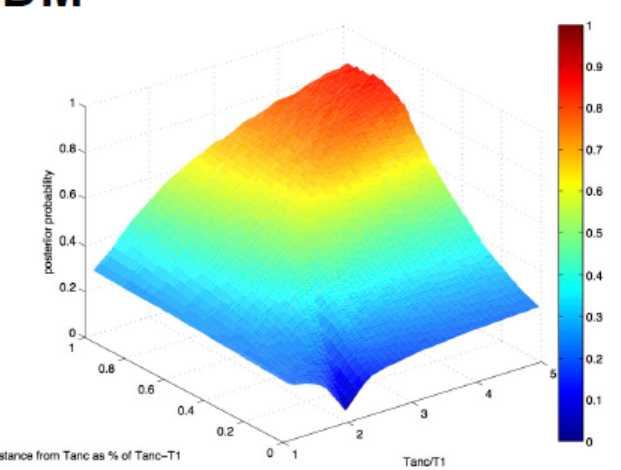

Tanc $=0.005$

5 Mya

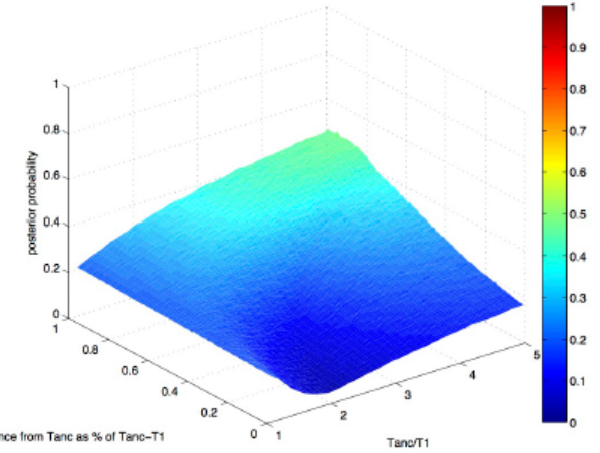

Tanc $=0.001$

1 Mya

Continued overleaf 
Asymmetric tree, $\theta=$ mixed DM
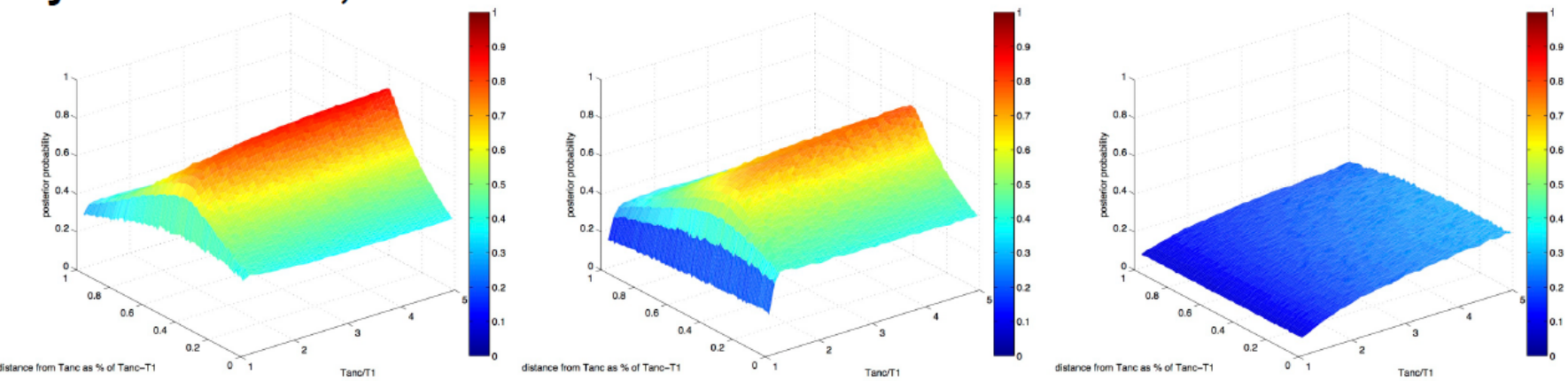

Symmetric tree, $\theta=$ mixed DM
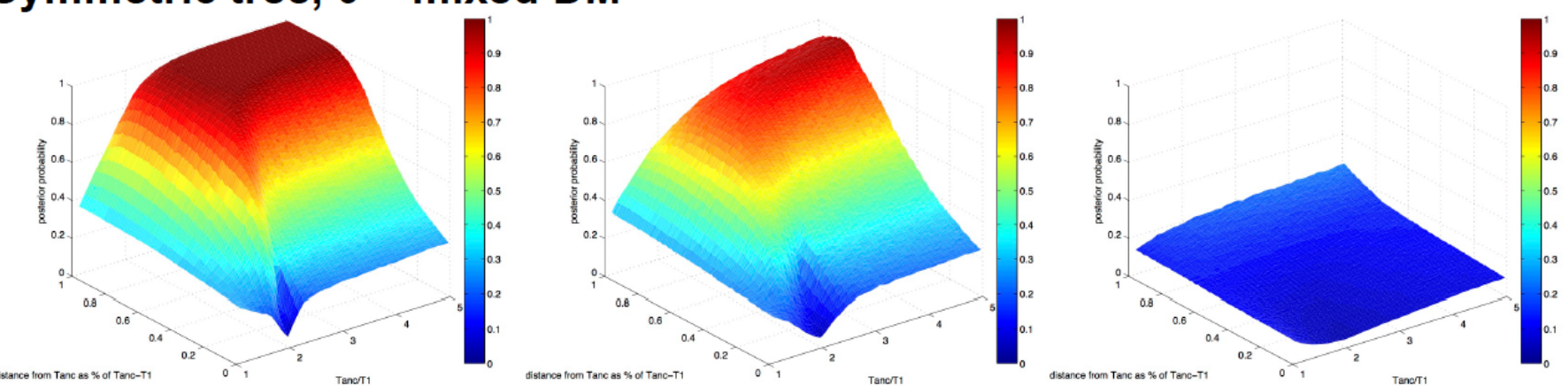

Tanc $=0.01$

10 Mya

Tanc $=0.005$

5 Mya
Tanc $=0.001$

1 Mya

Continued overleaf 


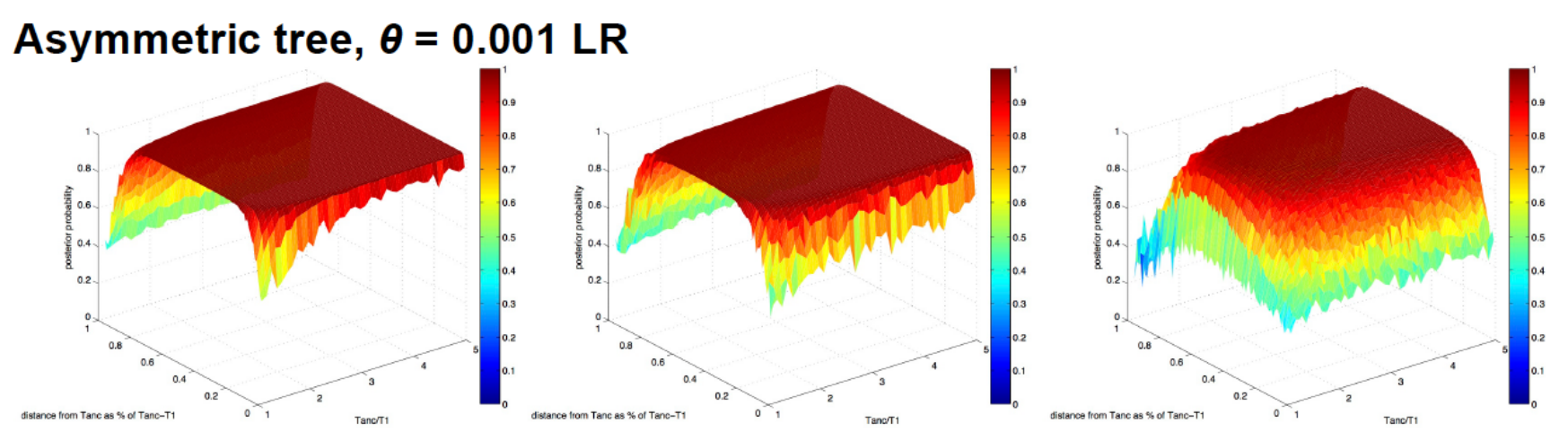

Symmetric tree, $\theta=0.001 \mathrm{LR}$

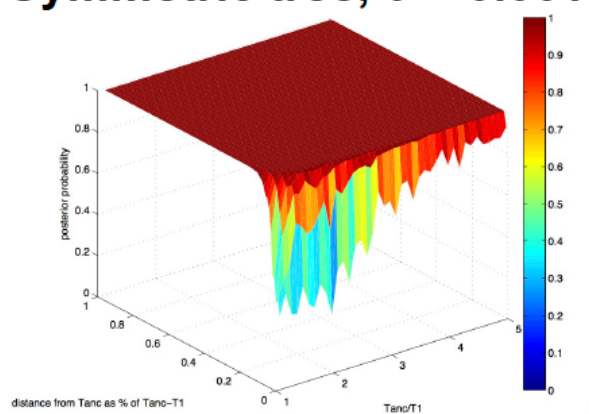

Tanc $=0.01$

10 Mya

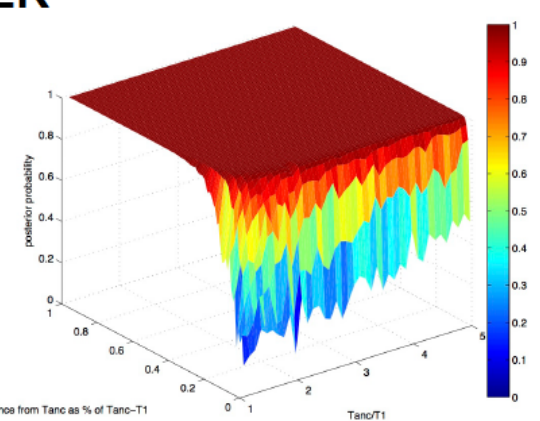

Tanc $=0.005$

5 Mya

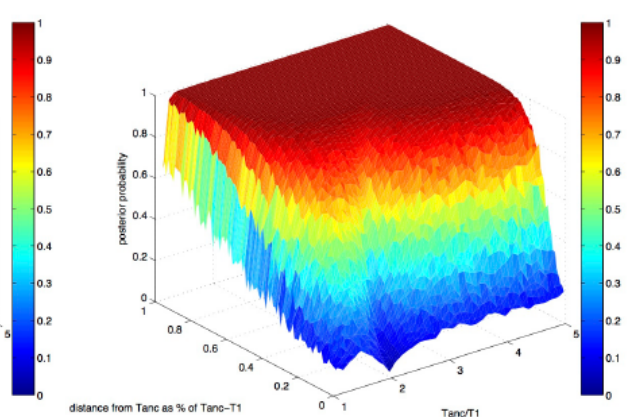

Tanc $=0.001$

$1 \mathrm{Mya}$

Continued overleaf 


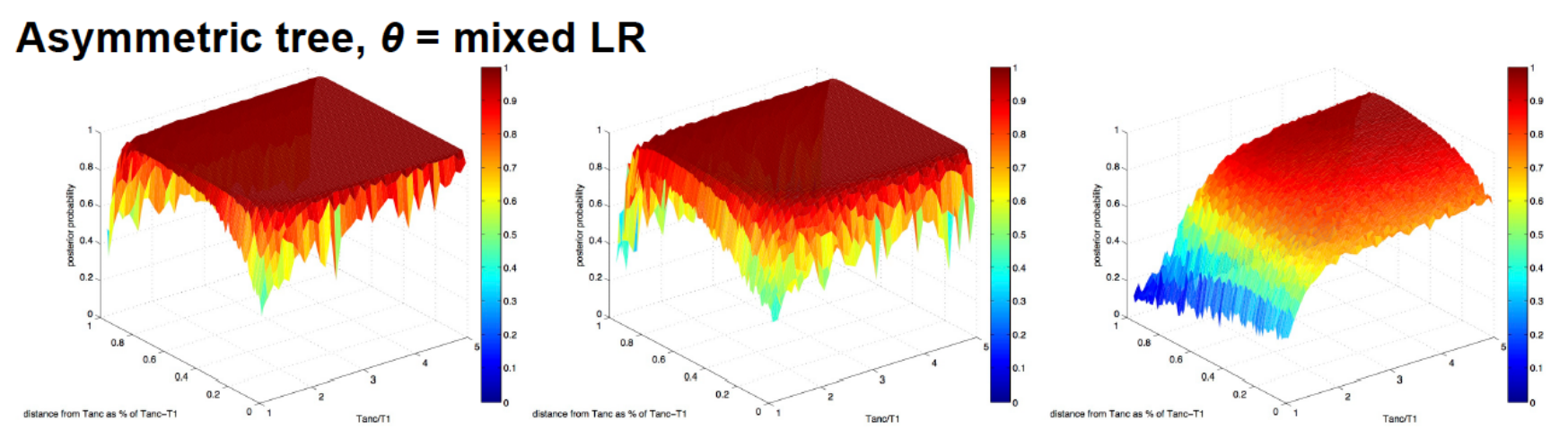

\section{Symmetric tree, $\theta=$ mixed LR}

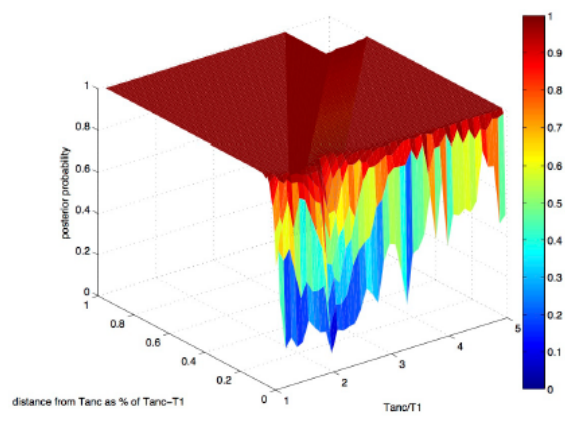

Tanc $=0.01$

10 Mya

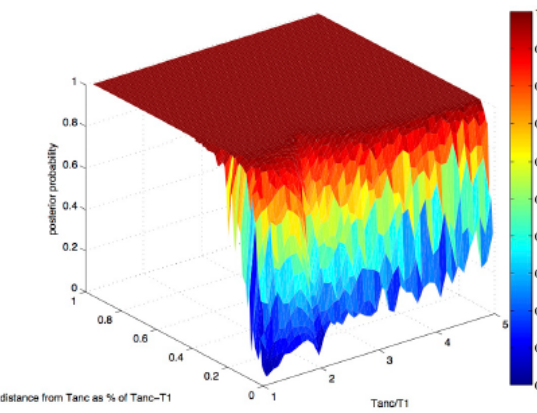

Tanc $=0.005$

5 Mya

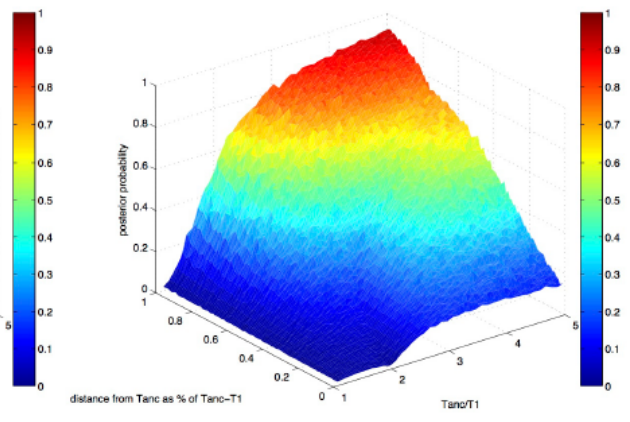

Tanc $=0.001$

1 Mya

Figure S5 Posterior probabilities of the true model (either an asymmetric or symmetric tree from a total of 15 possible models or topologies) as assessed by our ABC framework for a specific framework of demographic scenarios using the Direct (DR) and Logistic Regression (LR) methods. Tanc equal the total height of the tree. Conversion of height from substitutions per site to years is based on a mutation rate of $1 \times 10^{-9}$ per year. For $\theta=$ mixed, see Supplementary text for exact parameterization . 
A

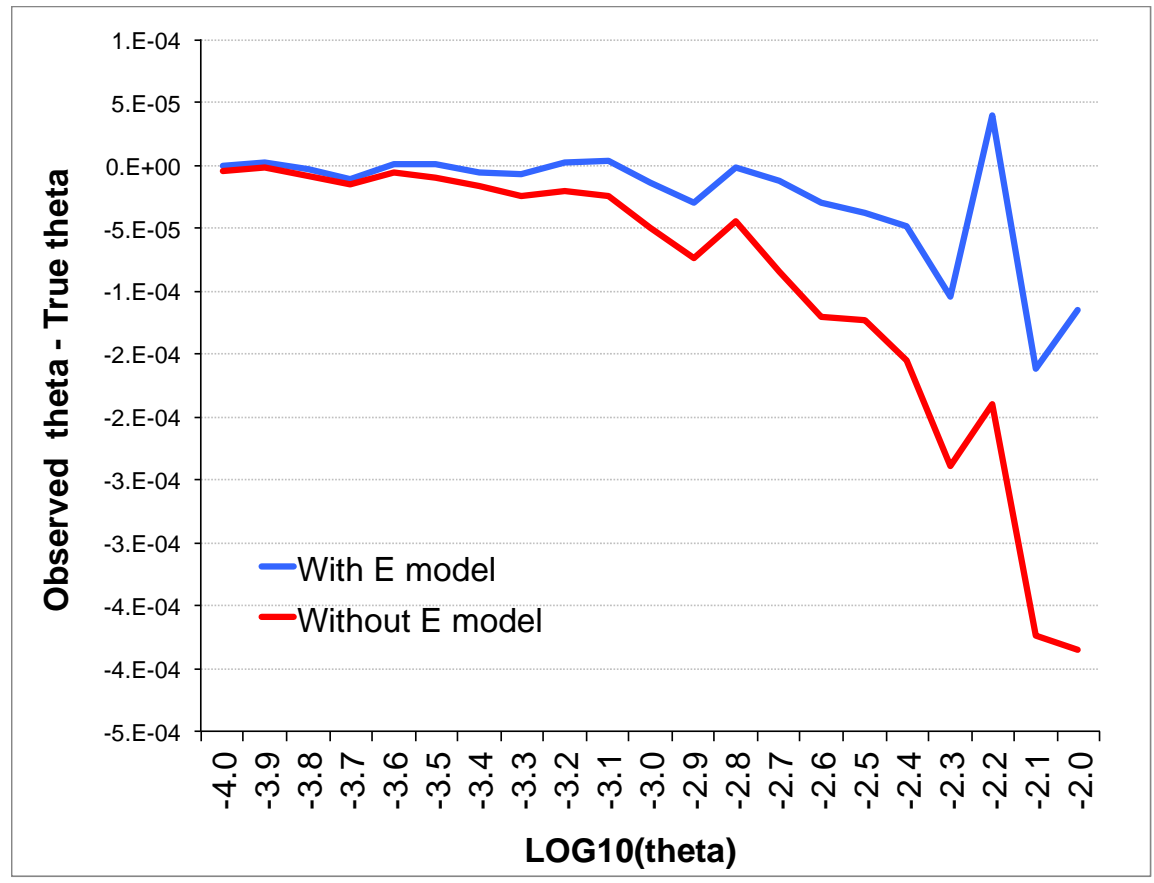

B

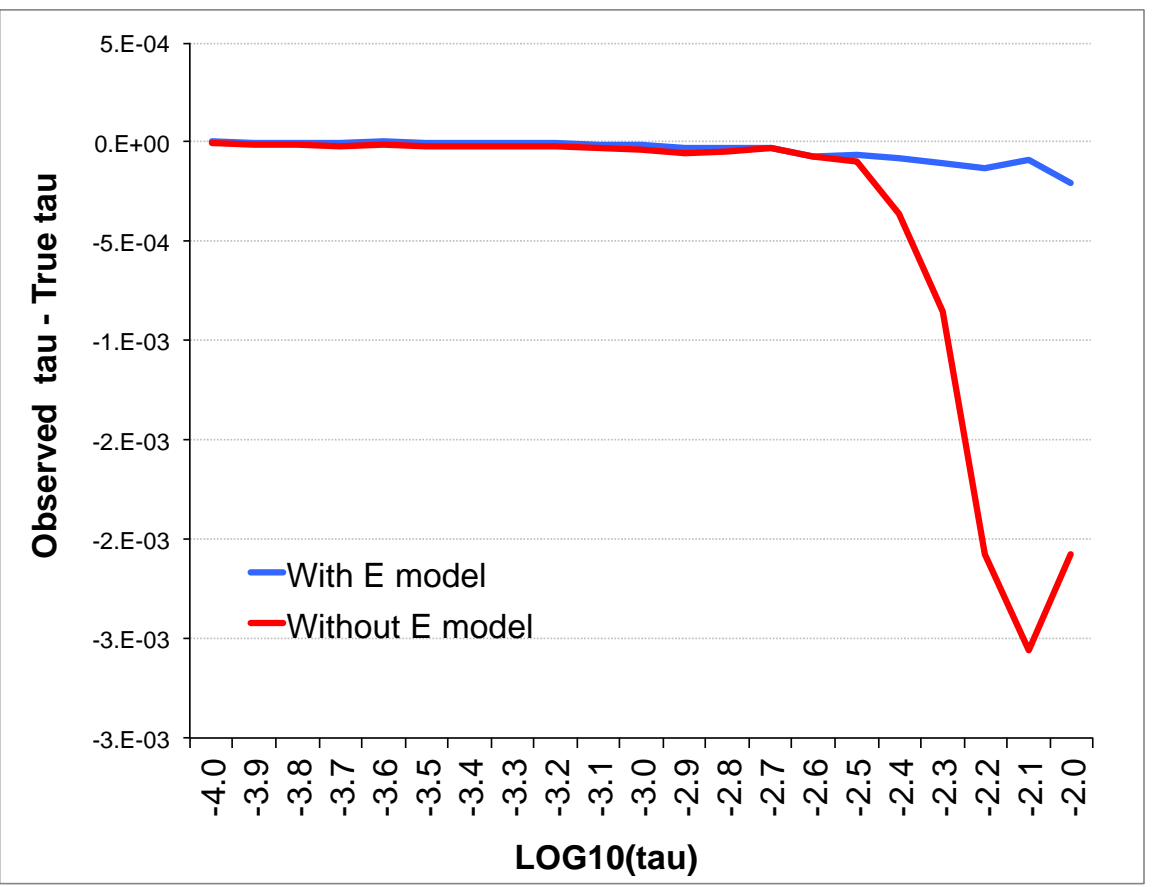

Figure S6 Comparison of observed versus true parameter value with and without stochastically modeling sequence errors using the $E$ model when estimating $\theta$ in scenario $A$ and $\tau$ in scenario $B$. 
A
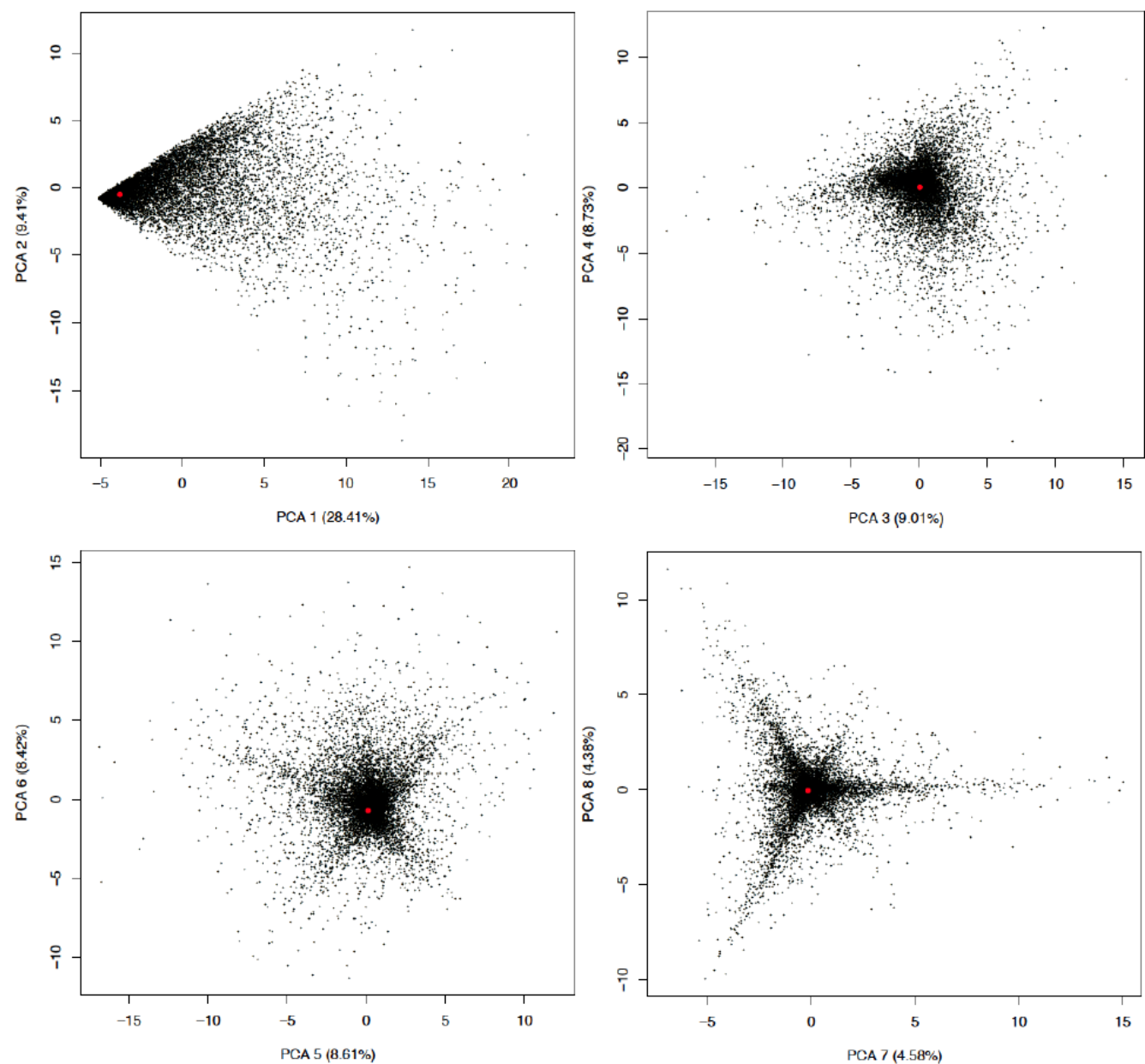

B
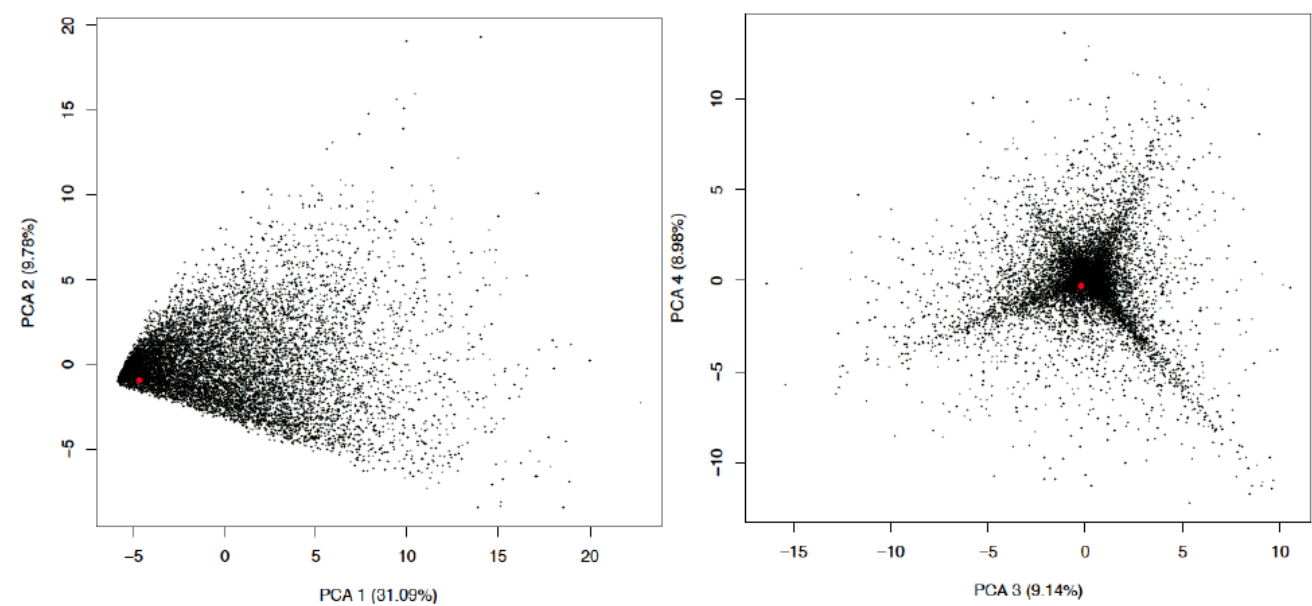

Figure S7 PCA of corrected simulated and observed (red) summary statistics for non-genic loci (A) and genic loci (B). 


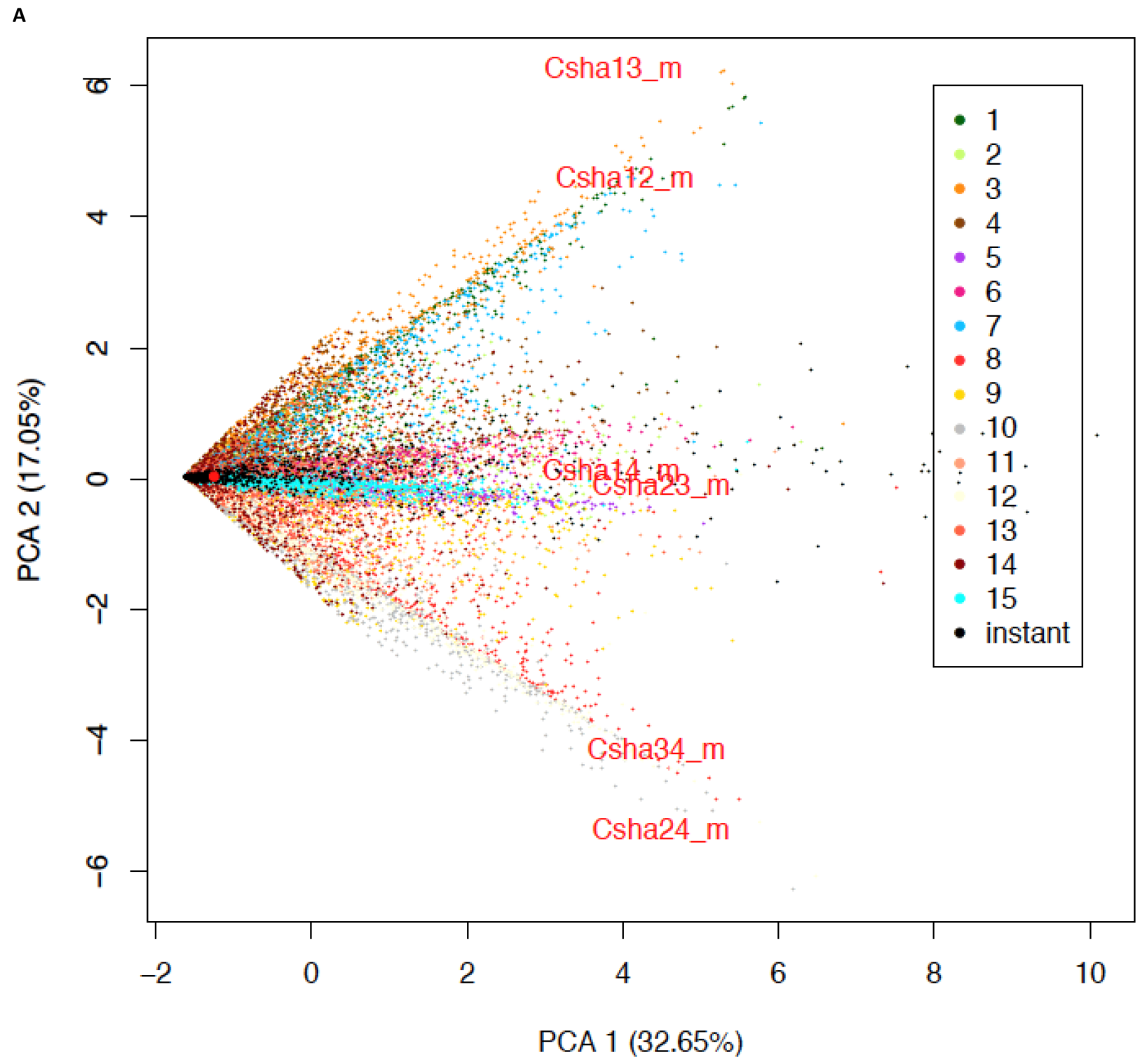




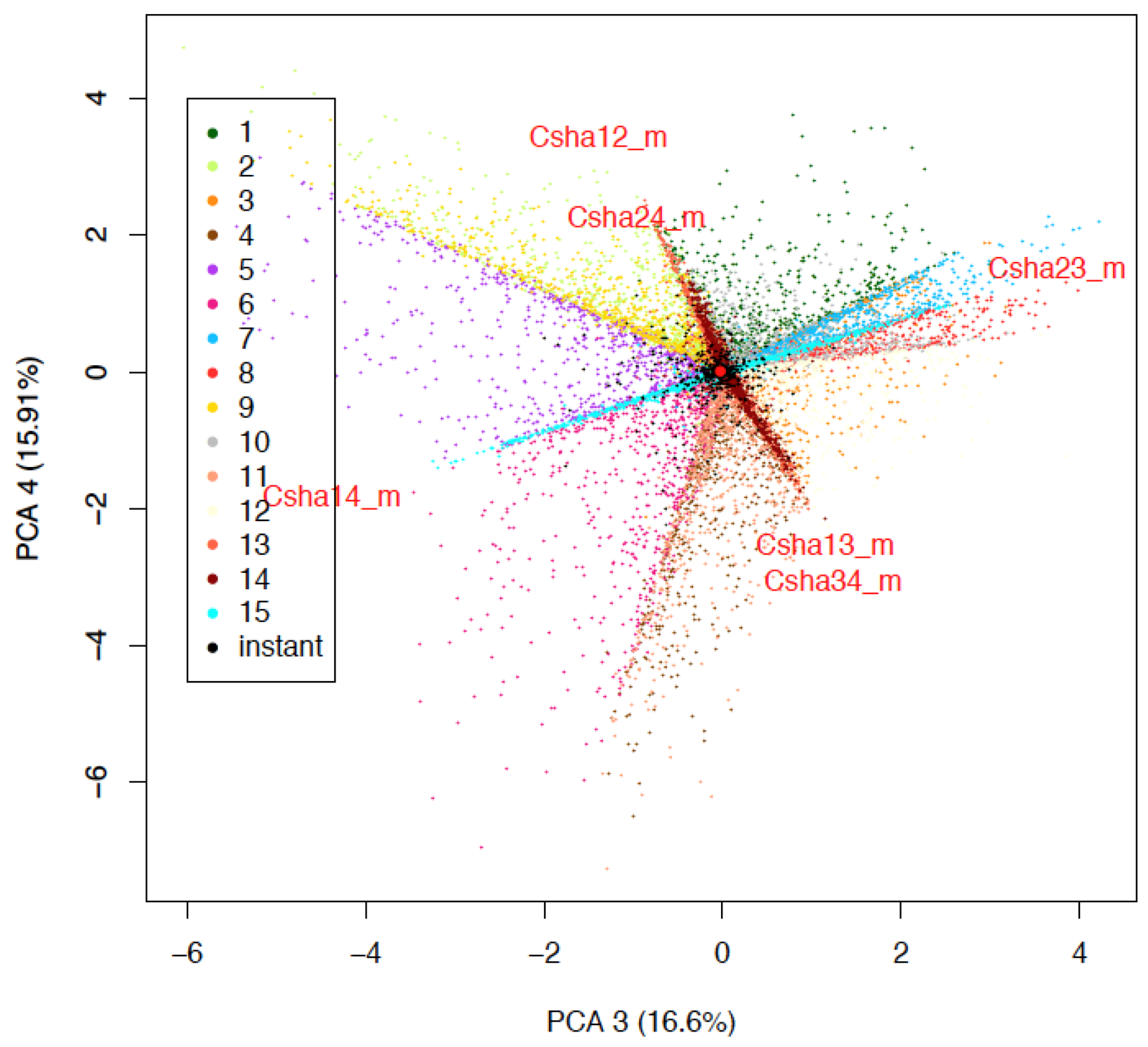




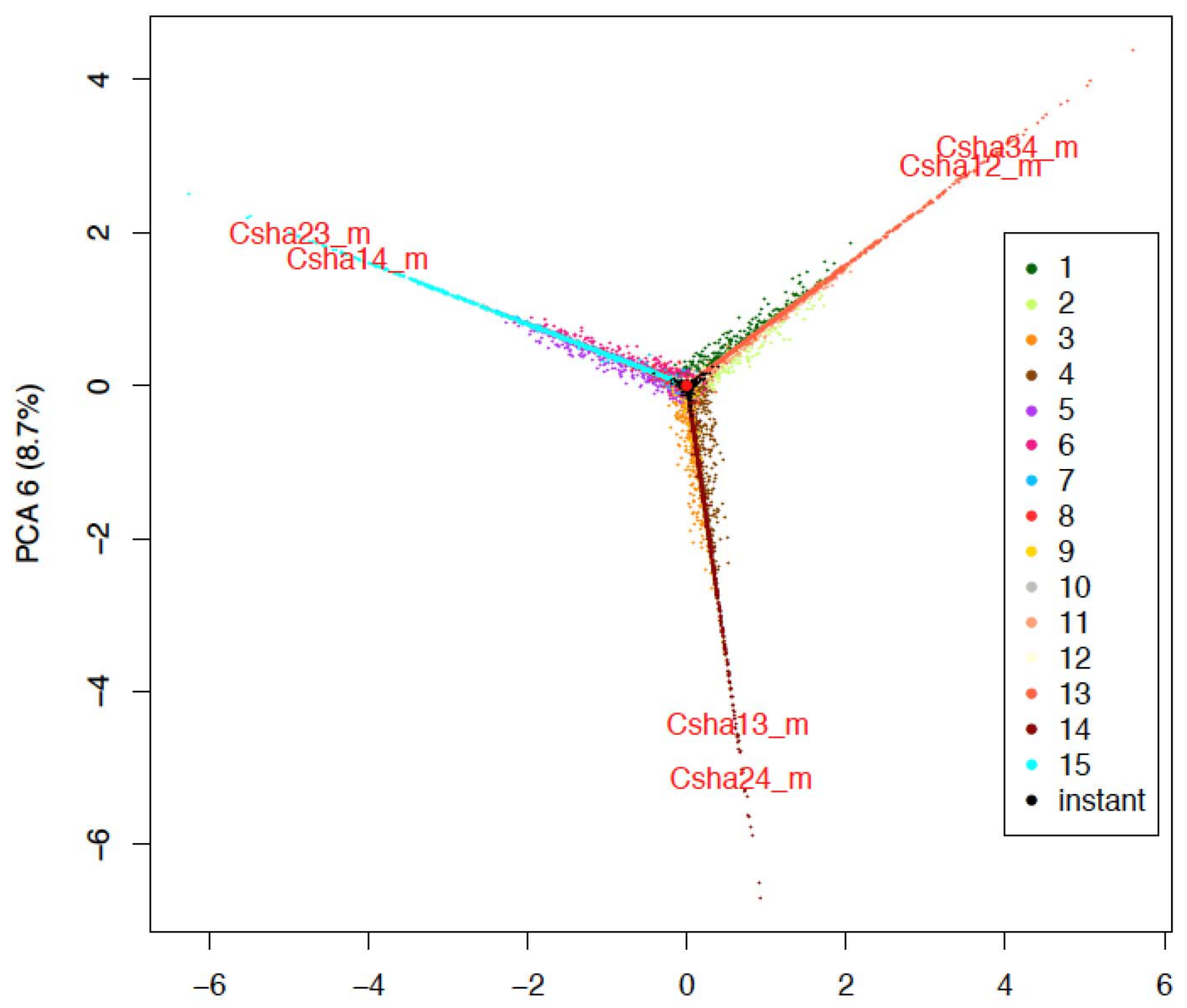

PCA 5 (9.08\%)

Figure S8 PCs 1 and 2 (A), 3 and 4 (B) and 5 and 6 (C) of 1,000 simulated sets of summary statistics (mean shared sites for all pairwise comparison) from each 15 bifurcating topology models and the 4-way hard polytomy model as well as the observed data in red for non-genic loci. Model numbers correspond to the topologies given in Table S12. The $E$ model was applied to these simulations. 

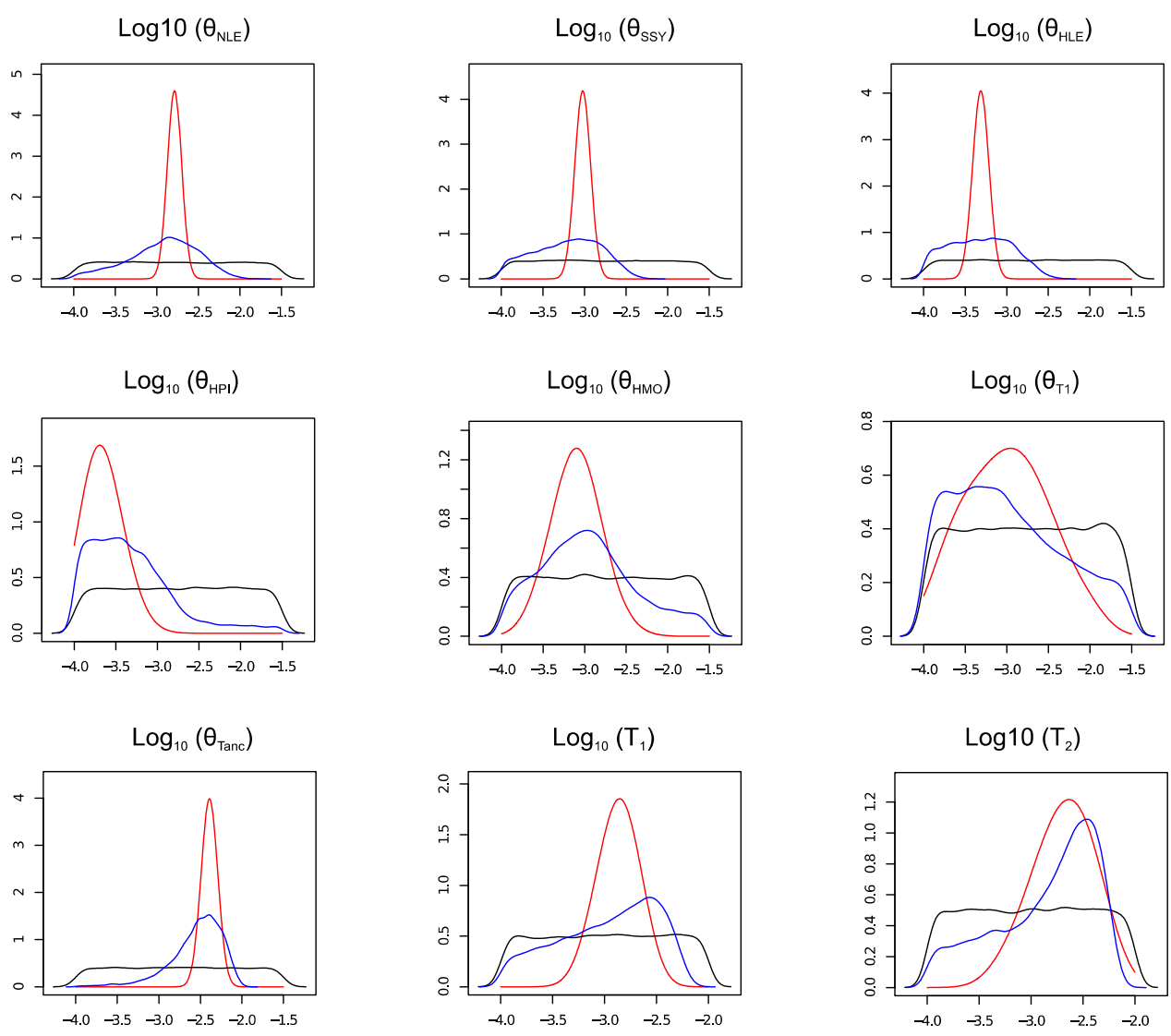

Figure S9 Posterior distributions for the instantaneous speciation model for gibbon genera 

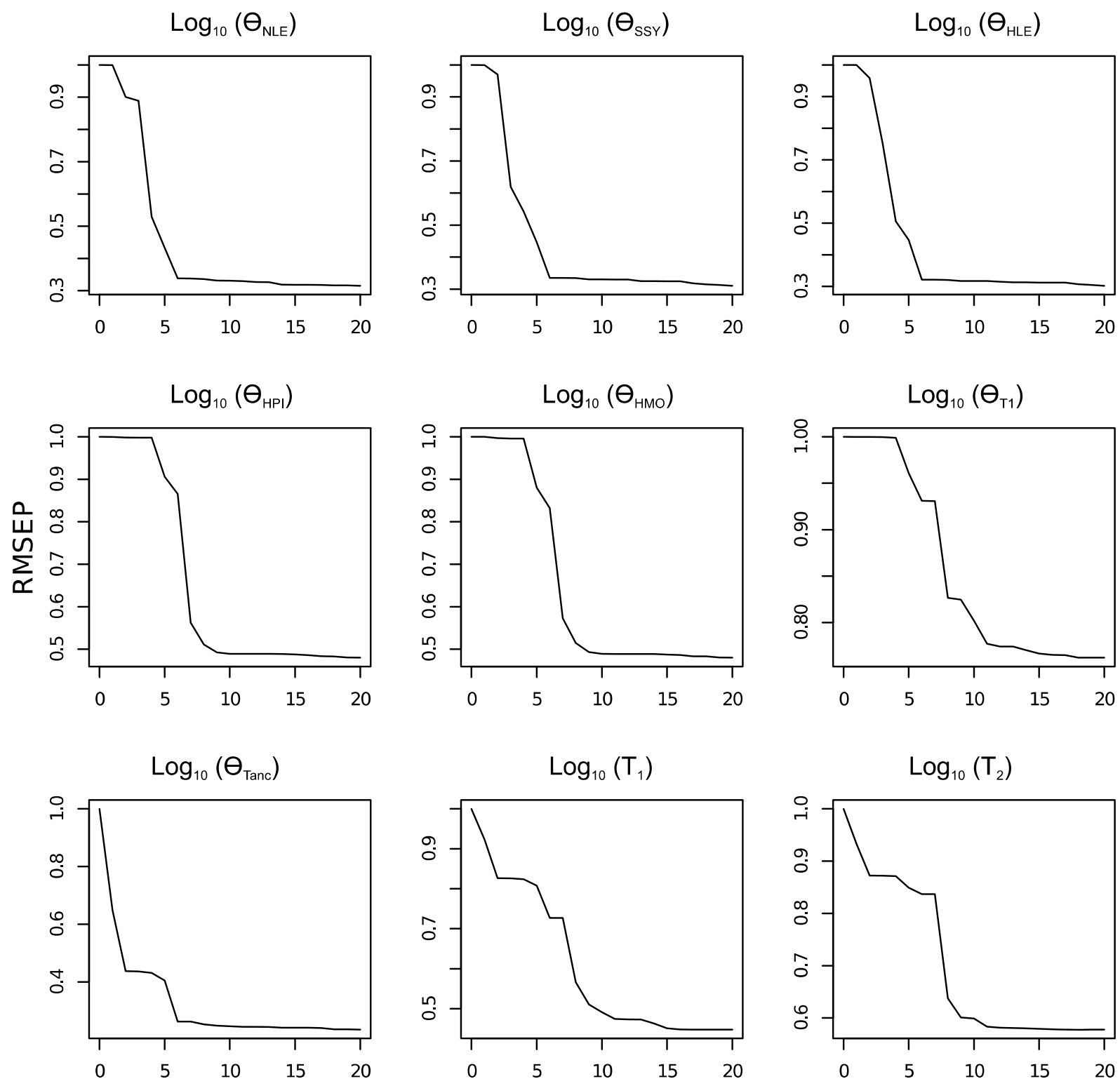

number of components

Figure S10 RMSE of PLS components for the instantaneous speciation model for gibbon genera 

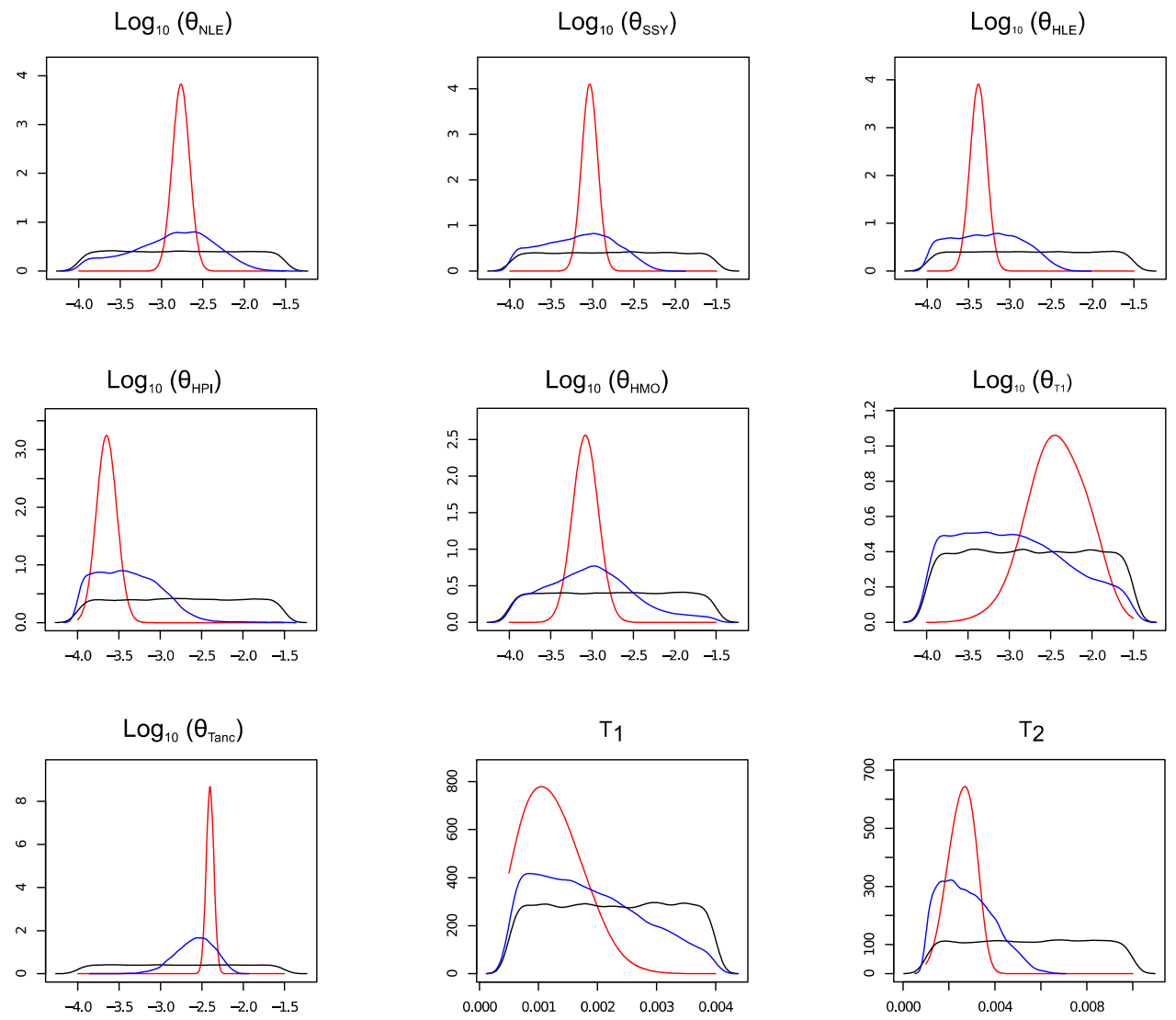

Figure S11 Posterior distributions for the instantaneous speciation model for gibbon genera using flat priors for $\tau$. 

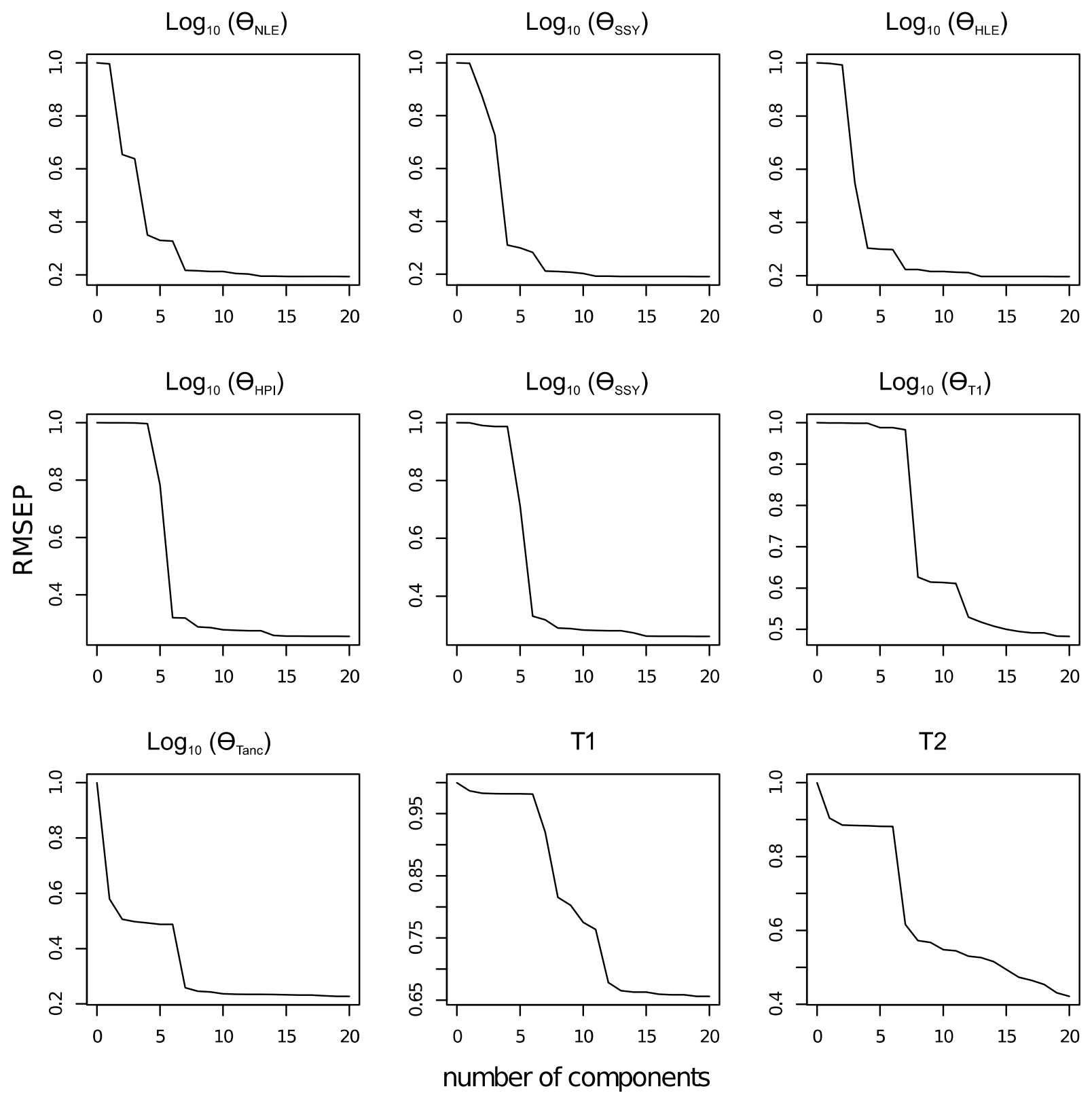

Figure S12 RMSE of PLS components for the instantaneous speciation model for gibbon genera using flat priors for $\tau$. 

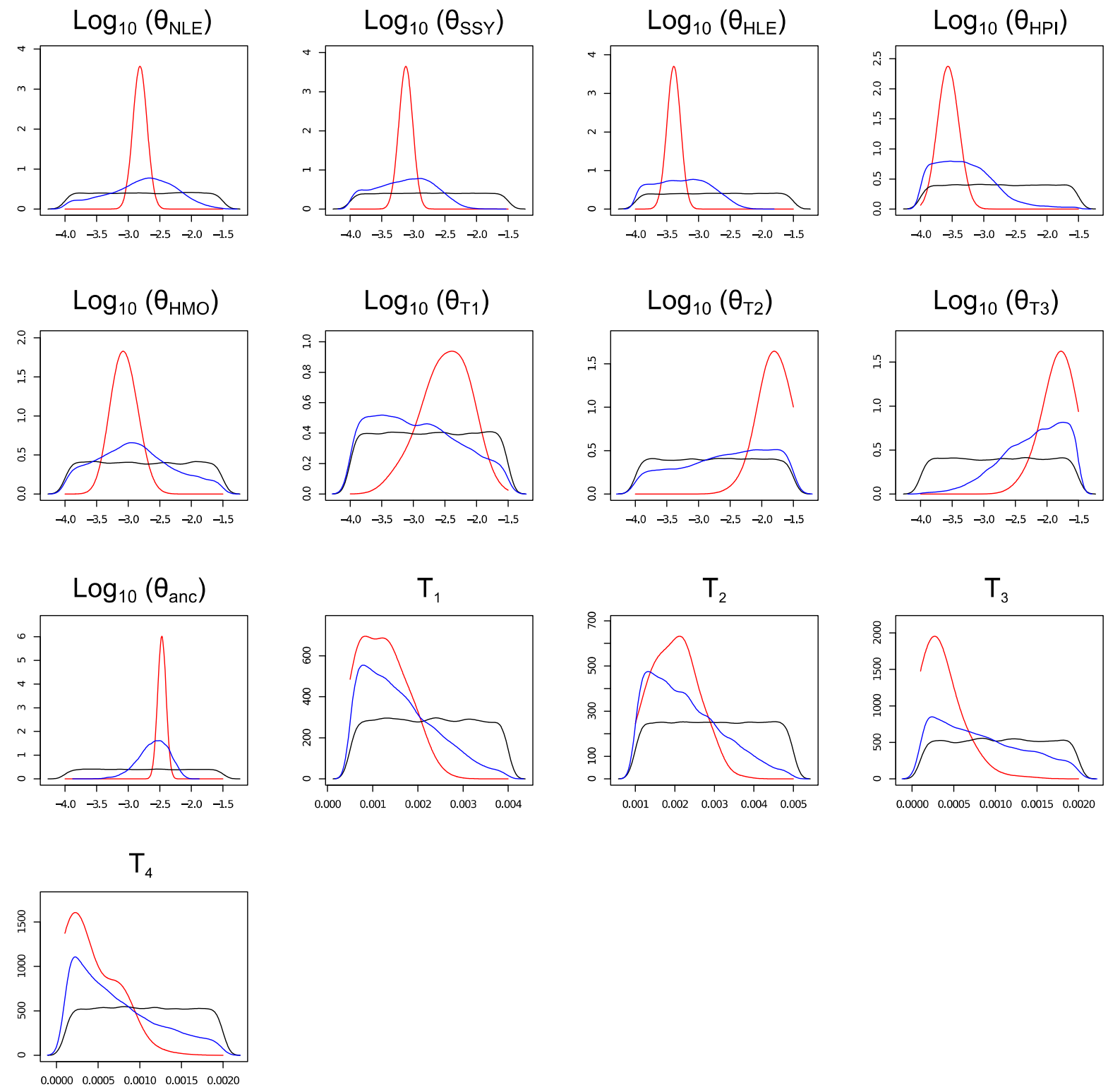

Figure S13 Posterior distributions for a bifurcating speciation model for gibbons genera. 

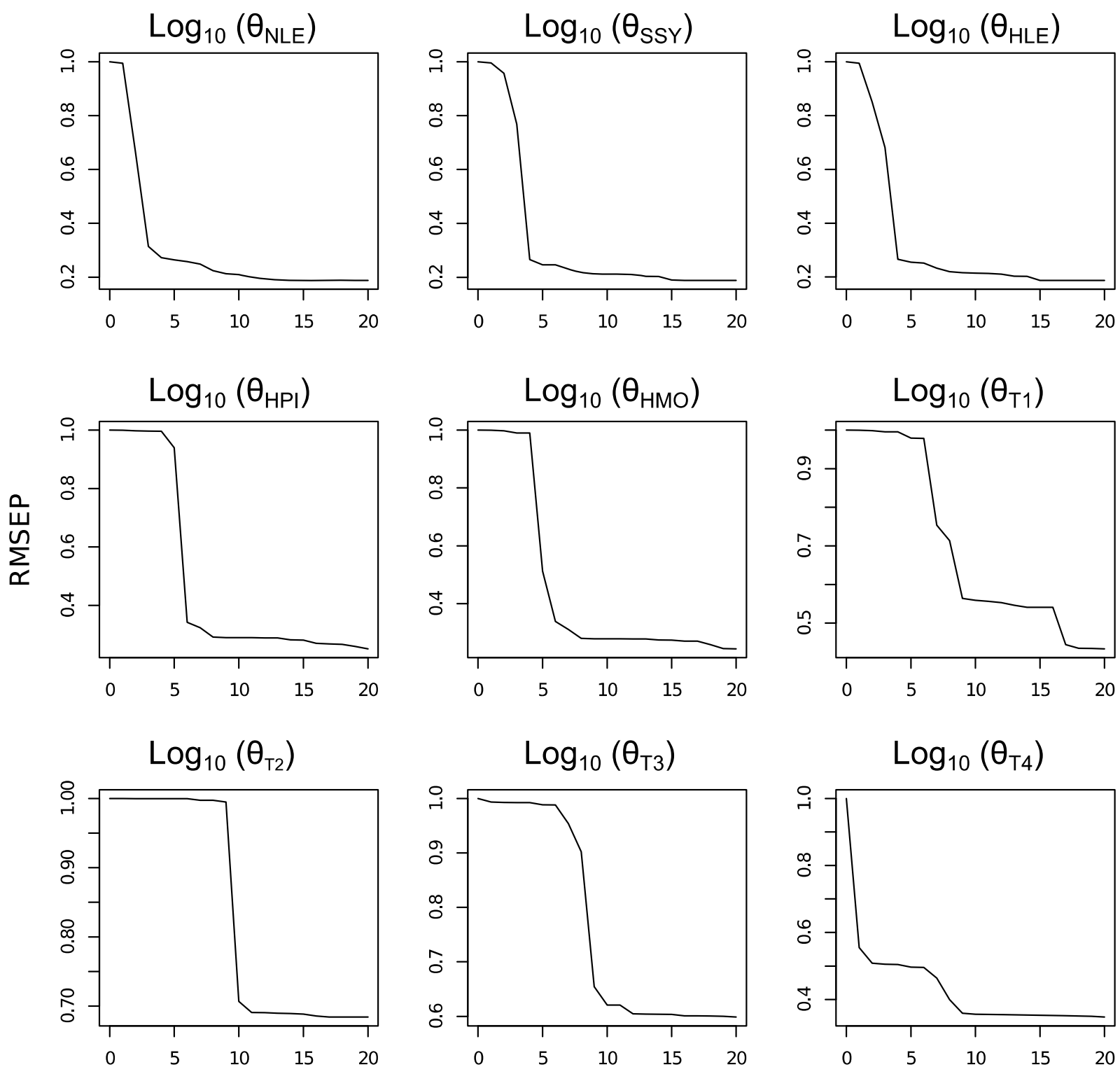

number of components 

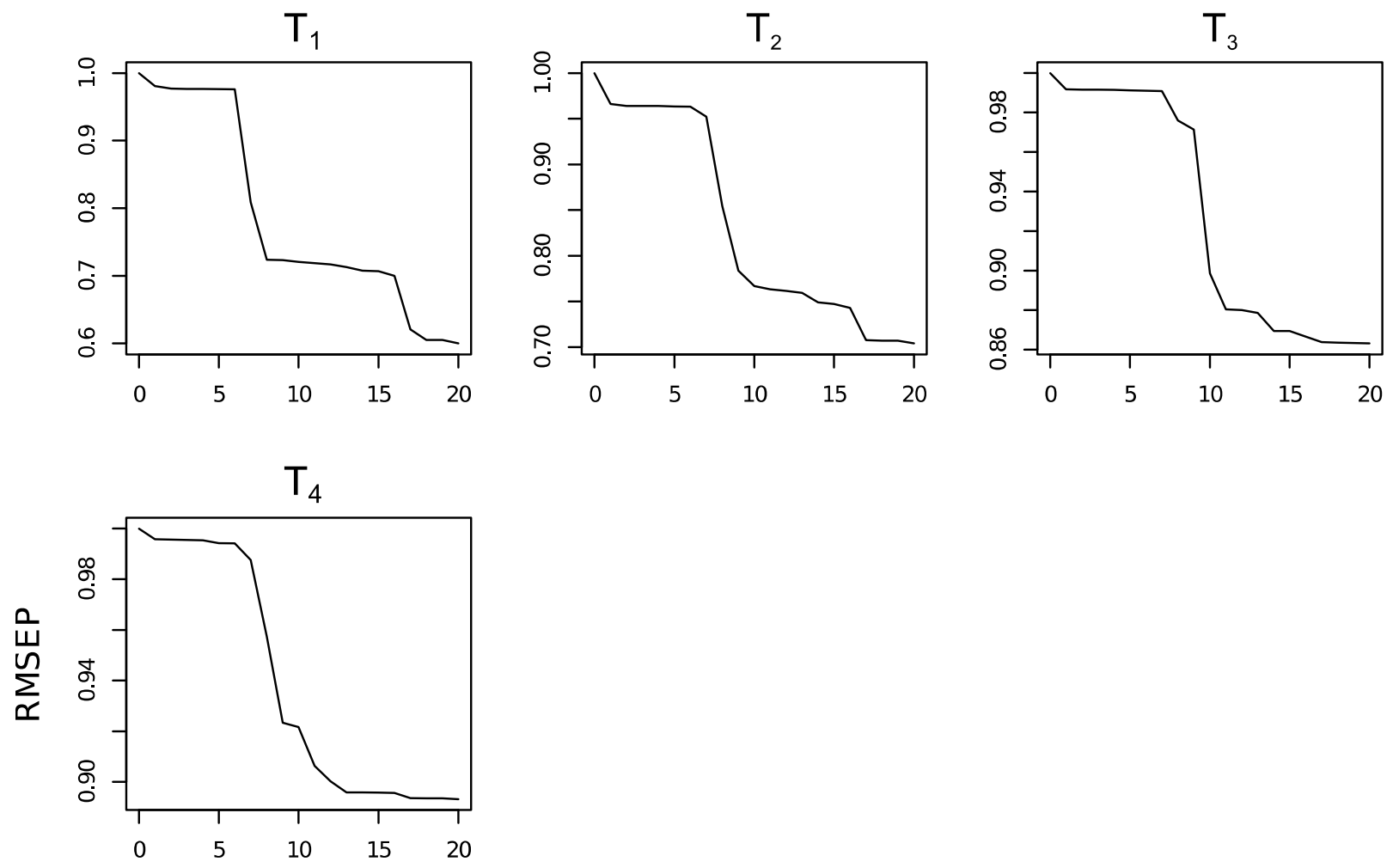

number of components

Figure S14 RMSE of PLS components for the bifurcating speciation model for gibbon genera for $\theta(A)$ and $\tau$ (B) parameters. 


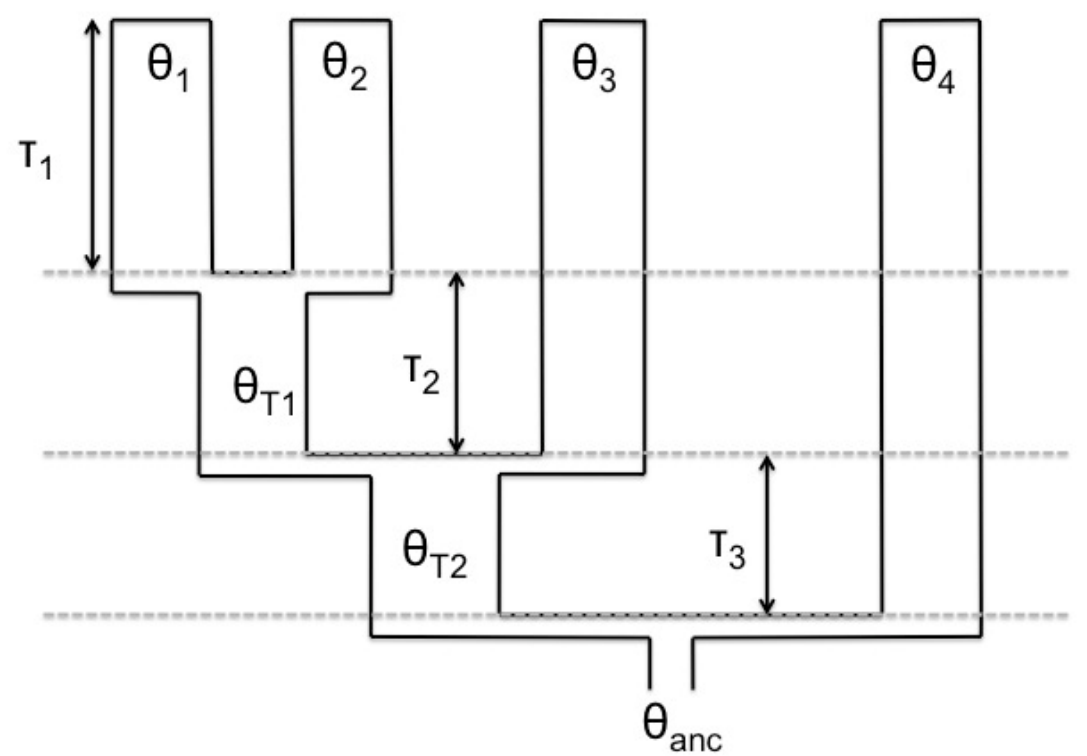

Figure S15 Example model setup for an asymmetric phylogeny 


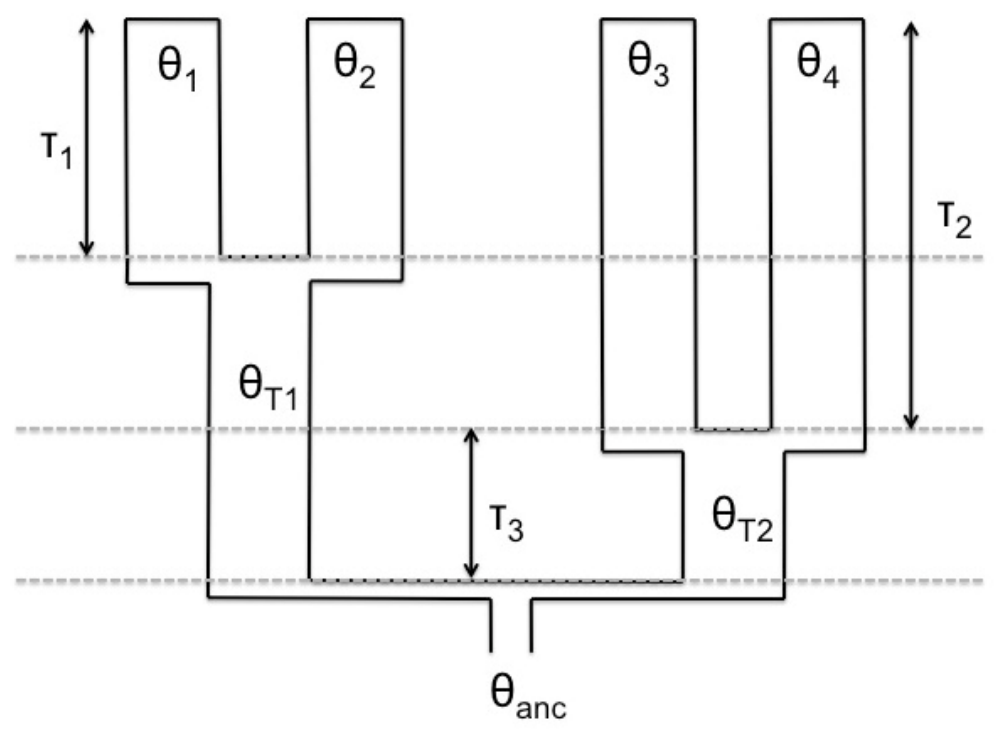

Figure S16 Example model setup for a symmetric phylogeny 


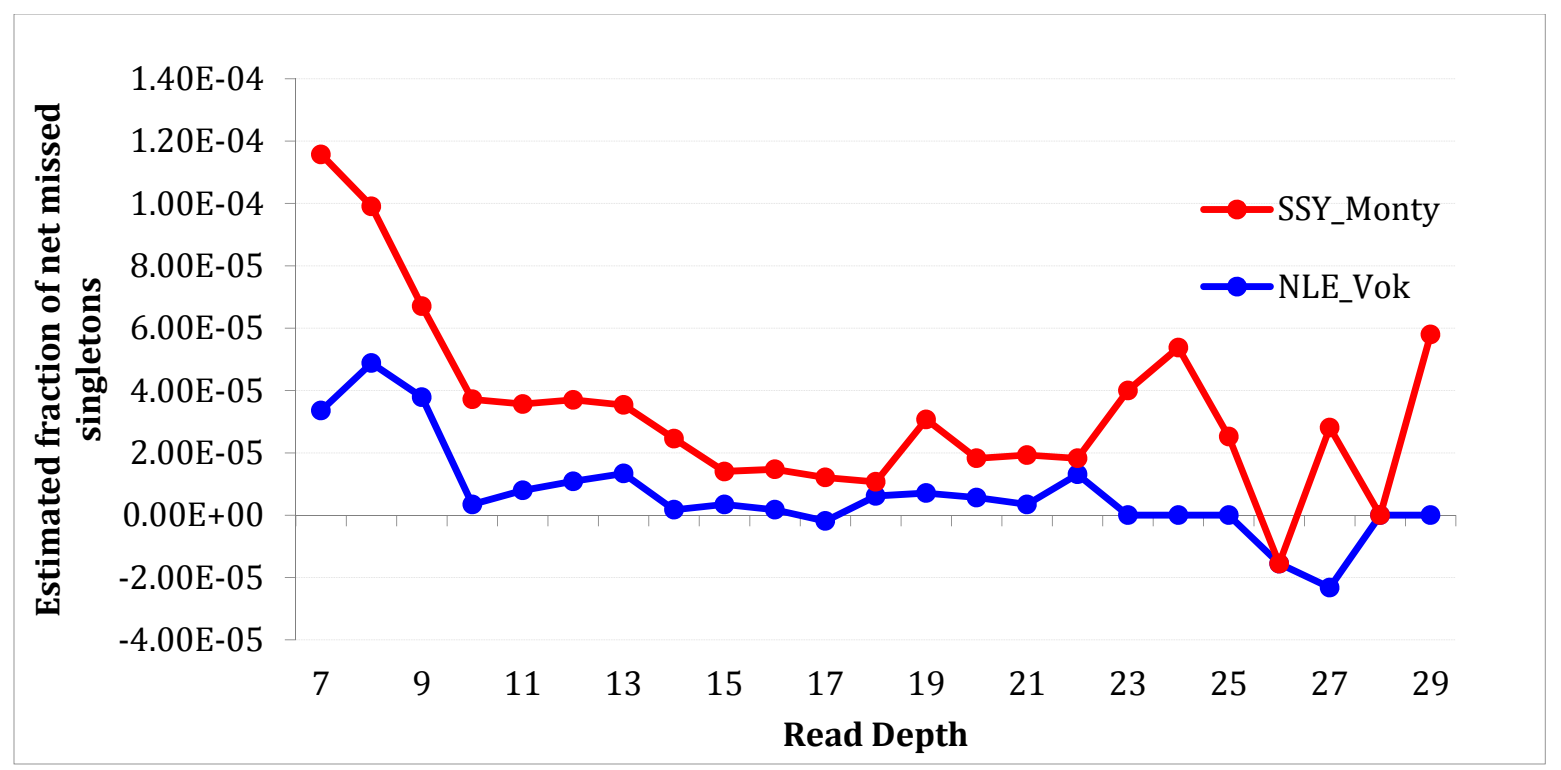

Figure S17 Net missed singletons per base, as a function of read depth. The net missed singletons per base is computed on the intersection of the exome-capture and the whole genome data, and is given as a function of the read-depth in the whole genome data. Due to sampling variance, there is modest variance in this function, but nevertheless error rates initially are high, then decrease, and then slightly increase again. 


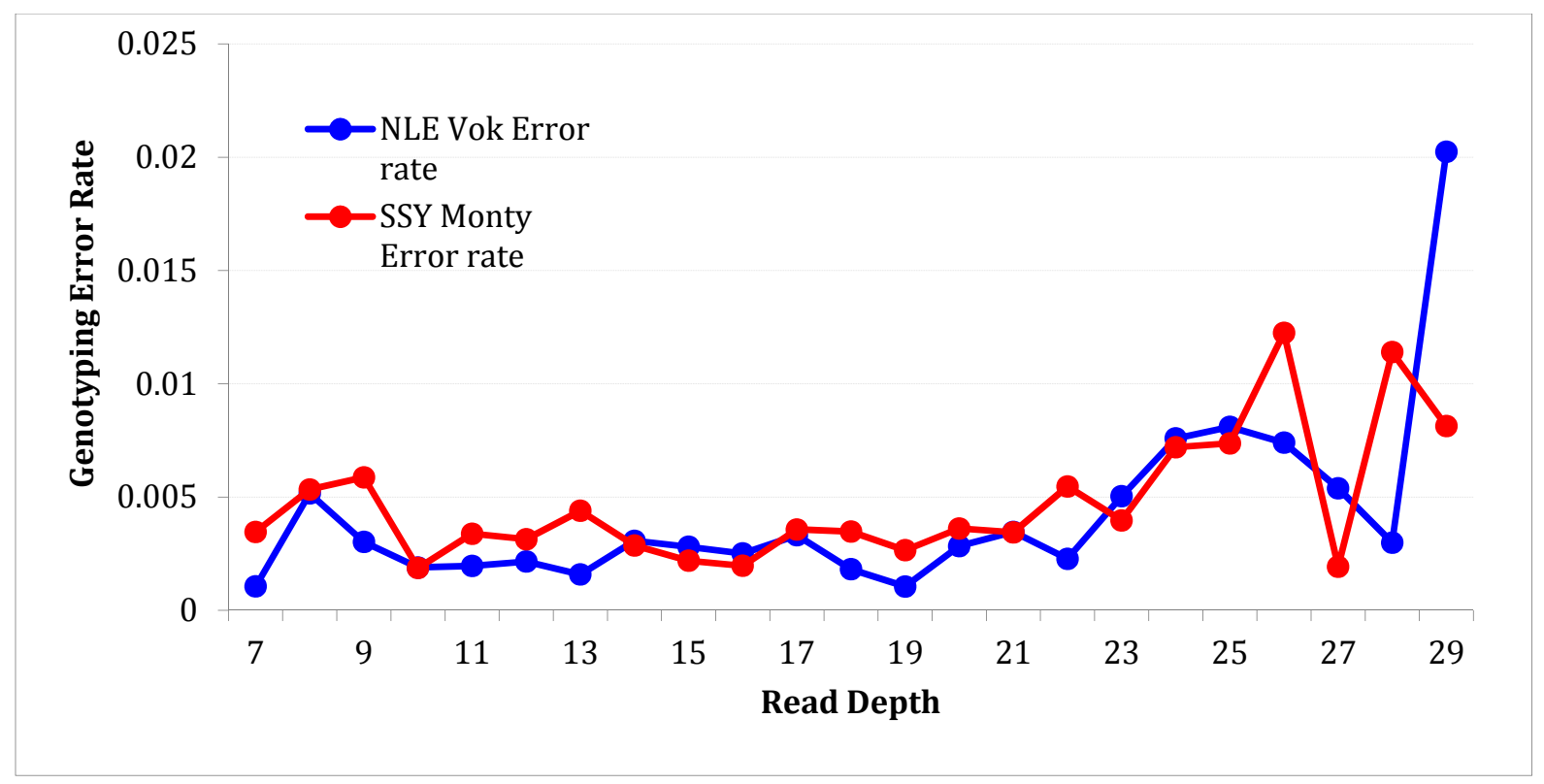

Figure S18 Genotyping error rates. For sites not called as singletons in the whole genome data, the error rate (defined as the probability of a miscall), is given as a function of the read-depth. Even after filtering CNV regions, error rates increase at high read-depth. 


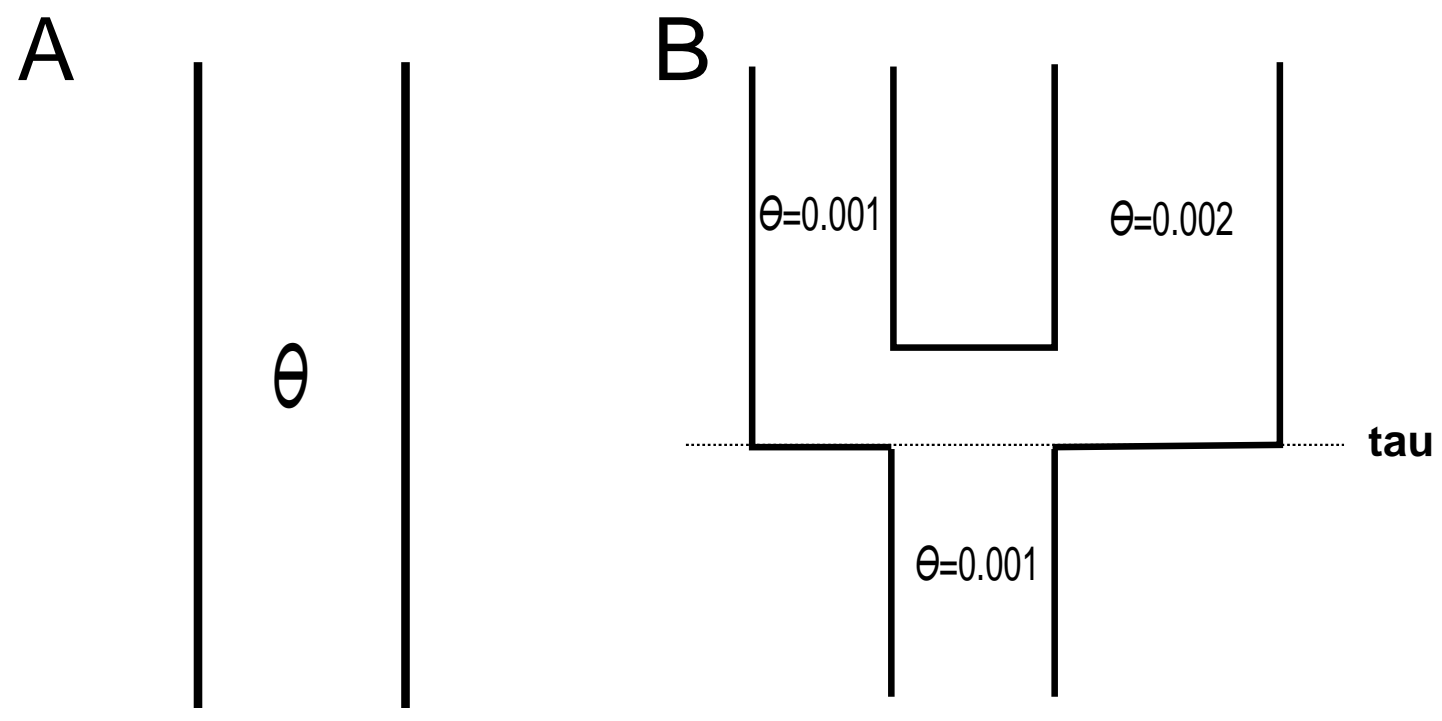

Figure S19 The two demographic scenarios, A and B, used to examine the effect of our E model for inferring $\theta$ and $\tau$. 
A

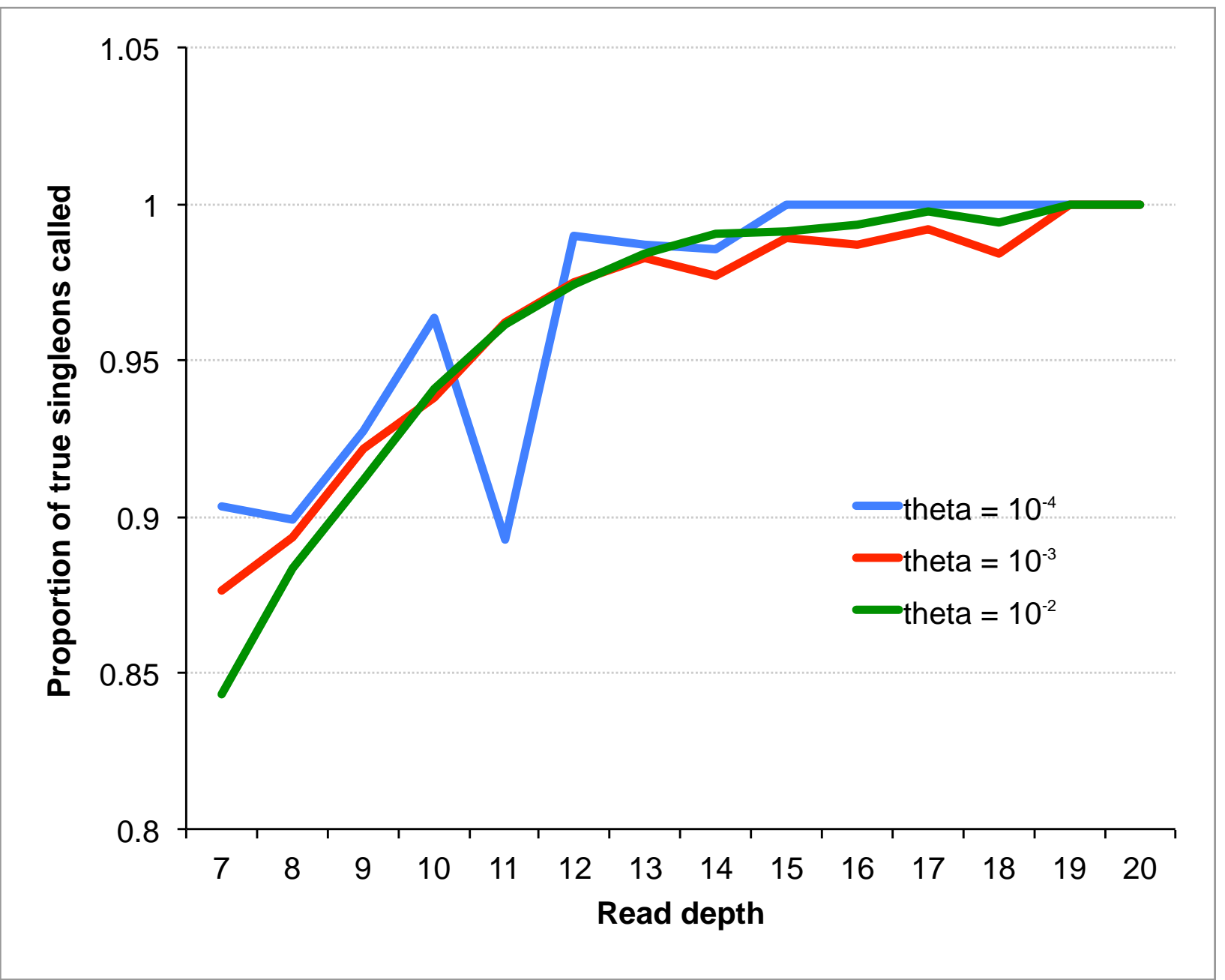




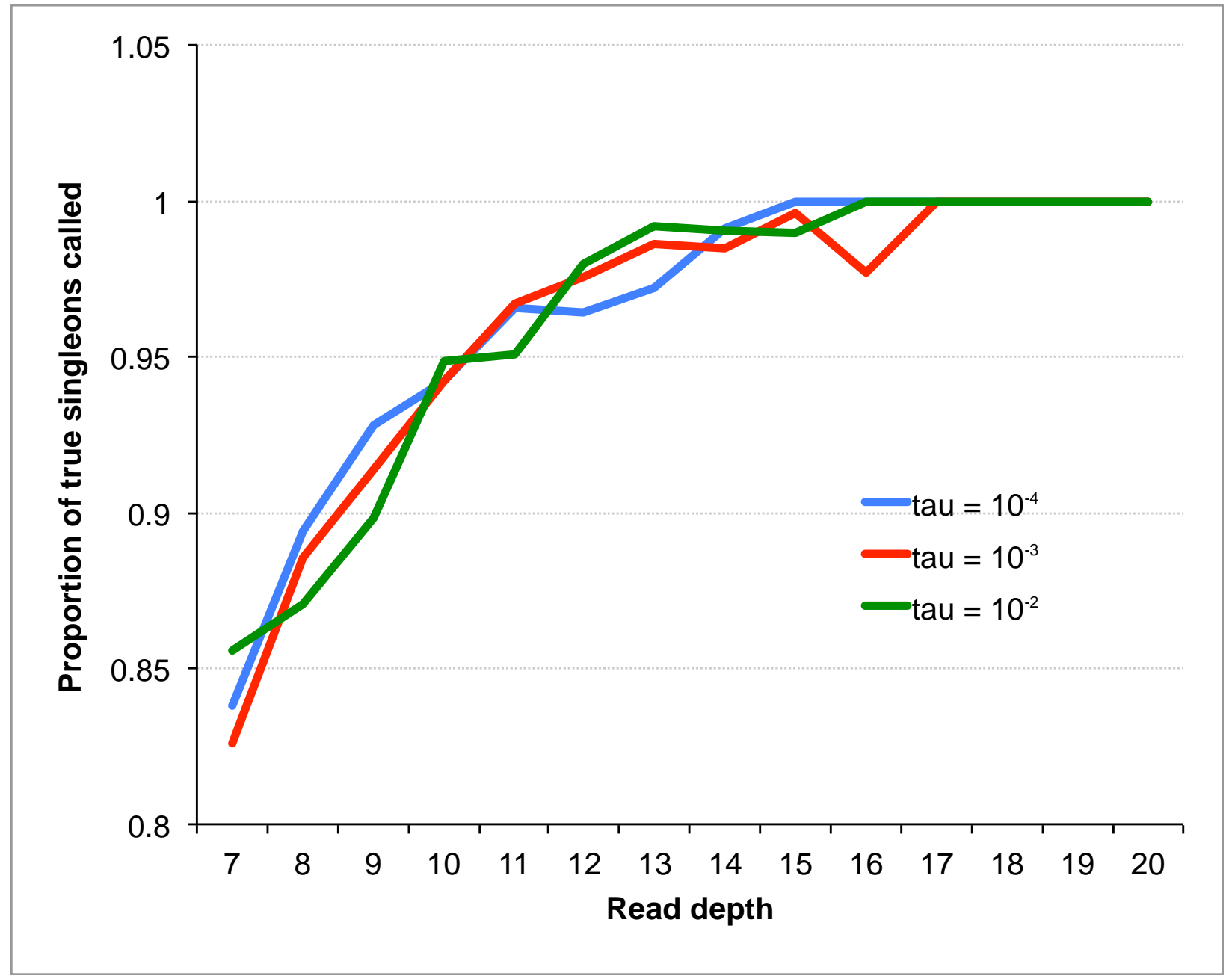




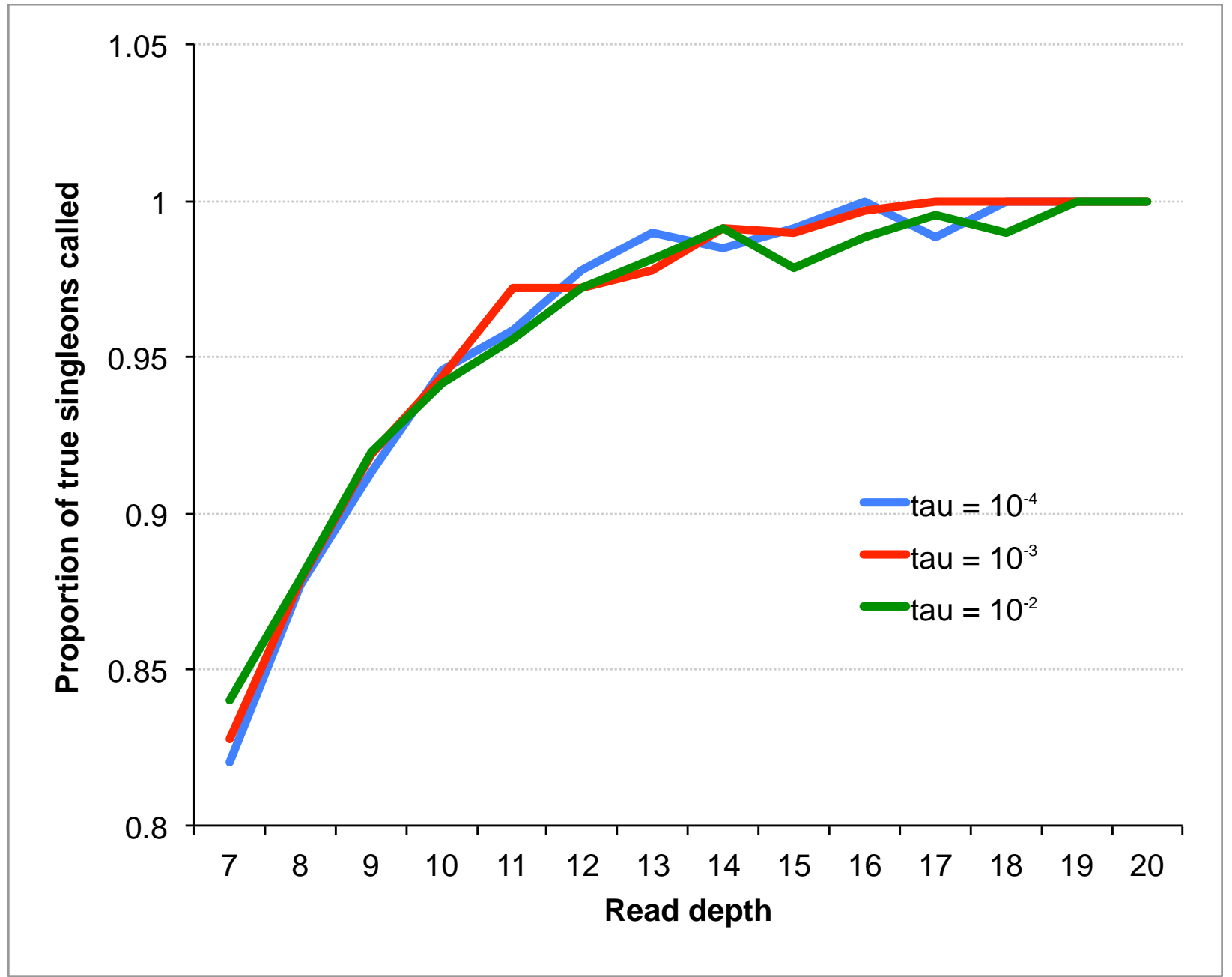

Figure S20 Proportion of true singletons called at a given read depth under scenario A for the reference target individual under three different $\theta$ values (A), under scenario $B$ for the reference target individual under three different $\tau$ values $(B)$ and under scenario $B$ for the non-reference target individual under three different $\tau$ values (C) 


\section{Tables S1-S12}

Available for download as Excel files at http://www.genetics.org/lookup/suppl/doi:10.1534/genetics.115.174425/-/DC1

Table S1: Priors and Posterior estimates for G-PhoCS analysis

Table S2: Concordance between genotype calls from whole genome (rows) and whole exome sequencing (columns) in the NLE and non-NLE individual. First two tables represent absolute number and second two tables represents cells as percentage of exome genotypes

Table S3: Posterior estimates for an instantaneous speciation model for gibbon genera for 15PLS components

Table S4: Posterior estimates for an instantaneous speciation model for gibbon genera for 10PLS components using a flat prior for $\tau$

Table S5: 95\% Cls for instantaneous speciation model examining the effect of ancestral state misidentification

Table S6: Posterior estimates for a bifurcating speciation model for gibbon genera

Table S7: Counts of allele sharing and D-statistic analysis for gibbon genera and species

Table S8: Summary of where sequencing of 8 gibbon samples was performed

Table S9: Summary of sequencing read post-processing

Table S10: Classifier accuracy using multiple ML methods

Table S11: Summary statistics used in $A B C$ analysis

Table S12: Key of numbers assigned to particular bifurcating topologies 